\title{
1 Generation of Vascularized Brain Organoids to Study Neurovascular Interactions
}

3 Xin-Yao Sun ${ }^{1,2,3, \#}$, Xiang-Chun $\mathrm{Ju}^{1,4, \#, *}$, Yang $\mathrm{Li}^{2}$, Peng-Ming Zeng ${ }^{2}$, Jian $\mathrm{Wu}^{2}$, Li-Bing

4 Shen ${ }^{1}$, Yue-Jun Chen ${ }^{1,3}$, Zhen-Ge Luo ${ }^{2, *}$

5

$6{ }^{1}$ Institute of Neuroscience, Center for Excellence in Brain Science and Intelligence

7 Technology, Chinese Academy of Sciences, Shanghai 200031, China.

$8{ }^{2}$ School of Life Science and Technology, ShanghaiTech University, Shanghai 201210,

9 China. ${ }^{3}$ University of Chinese Academy of Sciences, Beijing 100049, China.

$10{ }^{4}$ Present address: Okinawa Institute of Science and Technology, Onna-son, Japan.

11 \#These authors contributed equally to this work.

12 *Correspondence: luozhg@ shanghaitech.edu.cn; xiangchun.ju@ oist.jp

13 


\section{Abstract}

15 The recently developed brain organoids have been used to recapitulate the processes of

16 brain development and related diseases. However, the lack of vasculatures, which regulate

17 neurogenesis, brain disorders, and aging process, limits the utility of brain organoids. In

18 this study, we induced vessel and brain organoids respectively, and then fused two types

19 of organoids together to obtain vascularized brain organoids. The fused brain organoids

20 were engrafted with robust vascular network-like structures, and exhibited increased

21 number of neural progenitors, in line with the possibility that vessels regulate neural

22 development. Fusion organoids also contained functional blood-brain-barrier (BBB)-like

23 structures, as well as microglial cells, a specific population of immune cells in the brain.

24 The incorporated microglia responded actively to immune stimuli to the fused brain

25 organoids. Thus, the fusion organoids established in this study allow modeling interactions

26 between the neuronal and non-neuronal components in vitro, in particular the vasculature

27 and microglia niche.

Introduction

30 Recently, brain organoids derived from human pluripotent stem cells (PSCs), including

31 induced PSCs (iPSCs) and embryonic stem cells (ESCs), have been developed to model

32 developmental programs of human fetal brain, recapitulate developmental, psychiatric, and

33 degenerative brain diseases (Amin and Pasca, 2018; Di Lullo and Kriegstein, 2017; Kelava

34 and Lancaster, 2016; Lancaster and Knoblich, 2014). However, the lack of neurovascular

35 system, which is not only required for oxygen and nutrient supply, but also regulates

36 neurogenesis and brain functions (Delgado et al., 2014; Tata et al., 2016; Zhao et al., 2015;

37 Zlokovic, 2011), limits the applications of brain organoids. Thus, vascularization of brain

38 organoids represents one of the most demanded improvements in the field (Di Lullo and

39 Kriegstein, 2017; Giandomenico and Lancaster, 2017; Kelava and Lancaster, 2016;

40 Mansour et al., 2021). 
Blood vessels of the vertebrate brain are formed via sequential vasculogenesis and angiogenesis processes, which involve the initial invasion of endothelial cells (ECs) into the neuroepithelium regions via the perivascular plexus, their subsequent coalescence into primitive blood vessels, and growth and remodeling to form a mature vascular network (Lee et al., 2009). ECs are derived from the mesoderm-derived angioblasts (Zadeh and Guha, 2003). It has been shown that ECs can be derived in vitro from human PSCs, and these ECs can be potentially useful in engineering artificial functional blood vessels (Harding et al., 2017). However, the generation of complex vascularized organs from PSCs is still challenging, because it depends on the exquisite orchestration of cues from multiple germ layers and the gene expression profiles of ECs are controlled by finely patterned micro-environmental cues during organogenesis (Cleaver and Melton, 2003). Recently,

53 Wimmer et.al reported the generation of self-organizing human blood vessel organoids

54 induced from PSCs, and the application in the study of diabetic vasculopathy (Wimmer et 55 al., 2019). Given the mesodermal origin of ECs and ectodermic origin of neural fates

56 (Nostro et al., 2008; Stern, 2005), one barrier for the generation of vascularized brain organoids is the difficulty in simultaneous application of induction factors for distinct germ layers and cell fates due to mutual repression.

Several strategies have been used to establish the vascularized brain organoids. One strategy took advantage of natural angiogenesis of host blood vessels that sprout and grow into the grafted cerebral organoids, which later exhibited lower cell death rate and enhanced maturation (Mansour et al., 2018). Another approach utilized transcription factor-mediated differentiation of a subset of PSCs into EC-like cells during cerebral organoid induction, whose maturation process was also enhanced (Cakir et al., 2019). Two additional studies have tried co-culture with ECs or their progenitors during cerebral organoid formation (Lopez-Ramirez et al., 2019; Shi et al., 2020). Although grafted brain organoids appeared to have established functional blood vessels (Mansour et al., 2018), none of these methods can form an entirely and integrated vascular network in the cerebral organoids in vitro. And they all lacked the functional microglial cells, the only lifelong resident immune cells, 
71 which are derived from mesodermal origin (Muffat et al., 2016). In addition, the blood-

72 brain-barrier (BBB), the structure mainly composed of ECs, astrocytes and pericytes,

73 which protects the brain from circulation, is also lacking in the current ectodermal brain

74 organoid models. By co-culture of primary ECs, pericytes and astrocytes, the BBB

75 spheroids were created as an in vitro screening platform for brain-penetrating agents

76 (Bergmann et al., 2018; Cho et al., 2017).

Here, we develop an induction approach for brain-specific vascular organoids, which were cultured in medium containing neurotrophic factors at the maturation stage, to obtain cerebrovascular characteristics of the vessel organoids. Interestingly, a large number of microglial cells were induced by this approach along with other types of vascular cells. The

82 vessel organoids were then fused with the cerebral organoids in the Matrigel, leading to the 83 formation of vascularized brain organoids with invasion of microglia, which could be 84 activated upon immune stimuli. Thus, this study invents an advanced strategy that 85 incorporate vascular and microglia into brain organoids, providing a platform for the study 86 of interactions between neuronal and non-neuronal components during brain development 87 and functioning.

\section{Results}

\section{Generation of the Vessel Organoids (VOrs)}

91 It has been shown that the canonical Wnt signalling is required for development of ESC92 derived mesoderm (Lindsley et al., 2006) and the activation of Wnt signalling induces the 93 mesoderm differentiation from human PSCs (Nostro et al., 2008). Considering that EC94 generating vascular progenitors (VPs) are derived from mesoderm during embryogenesis 95 (Gupta et al., 2006), we performed guided mesodermal induction of H9 human embryonic 96 stem cells (hESCs), followed by endothelial differentiation. First, we treated 2-day old (D2)

97 embryonic bodies (EBs) from hESCs, which stably expressed GFP, with GSK3 inhibitor 98 CHIR99021 to activate the canonical Wnt signalling for mesoderm induction (Figure 1A). 
After 2 days, the EBs were treated with basic fibroblast growth factor (bFGF), vascular endothelial growth factor (VEGF), and bone morphogenetic protein 4 (BMP4), all of which have been shown to be able to promote VP differentiation into ECs (Cai et al., 2012; Jih et al., 2001). After 3 days, the differentiated ECs were incubated with endothelial medium ECGM-MV2 (shorted as MV2 hereafter) containing VEGF for 5 days for further maturation, and then embedded in Matrigel droplets. At late maturation stages, neurotropic reagents $\mathrm{N} 2$ and $\mathrm{B} 27$ were added into the maturation medium, which presumably might be able to induce some cell types with specific brain-vessel features (Figure 1A). Notably, vessel-like structures sprouted out from the spheroids (Figure 1B), reminiscent of initial vasculogenesis and angiogenesis. Remarkably, the vessel organoids (VOrs) showed gradual increase in the size during the maturation stage after day 16 (D16), with apparent tubular network characteristics (Figure 1-figure supplement 1A and B).

To verify the cell fates in developing VOrs, we performed quantitative PCR to determine the expression of stemness or vascular-specific genes at different time upon organoid differentiation. As shown in Figure 1C, the stemness markers (NANOG, OCT4) showed marked decrease 2 days upon mesoderm induction (D4), whereas the vessel markers (PECAM1, VE-cadherin, VWF, VEGFR1, VEGFR2 and PDGFR $\beta$ ) markedly increased after VP differentiation (D7 and thereafter). In line with this, flow cytometry results revealed the appearance of $\mathrm{GFP}^{+} \mathrm{CD} 31^{+} \mathrm{ECs}$ after D7, indicating the induction of the endothelial cells (Figure 1-figure supplement 1C).

Morphologically, $\mathrm{CD}^{+} 1^{+} \mathrm{ECs}$ in VOrs at D40 showed integrated and complex structures (Figure 1D), and exhibited remarkable vascular branches and tips undergoing angiogenesis-like processes (Figure 1E and Figure 1-figure supplement 1D). The 3D reconstructed cross-section revealed tubular structures in VOrs, reminiscent of vessel lumen (Figure 1E, see top right e1). To determine the connectivity and integrity of vessellike structures, we injected fluid into the lumens and observed that the hydraulic pressure caused liquid flow and vessel wall expansion without leakage (see Movie Supplement 1). 
Notably, PDGFR $\beta$-labeled pericytes that are believed to regulate EC maturation, stabilize vessel wall, and control angiogenesis, were also observed in close contact with ECs undergoing vessel differentiation (Figure 1F). For more details about the vasculature morphology, we used the Angiotool software which had been used as a tool for quantitative vessel analysis (Zudaire et al., 2011). The average vessel length was around $400 \mathrm{~mm}$, the vessel lacunarity was 0.15 , and the total number of junctions was about $700-800$ per VOr (Figure 1-figure supplement 1E).

To assess the function of ECs in VOrs, we determined the ability to incorporate DiIacetylated low density lipoprotein (DiI-Ac-LDL), as shown in a previous study (Lehle et al., 2016). We found that VOrs after D14 already had the ability of up-taking DiI-Ac-LDL, whereas ESCs could not (Figure 1-figure supplement 1F). Thus, we have successfully established a fully structured and functional vessel organoid model.

\section{Cell composition of brain-specific VOrs resembles brain vessels in vivo}

The vessel system of the brain contains a variety of vascular cell types (Vanlandewijck et al., 2018). To investigate the fidelity of VOrs in recapitulating the cerebrovascular cell types, we performed single-cell RNA sequencing (scRNA-seq) of VOrs at D40 using 10xGenomic chromium system (Macosko et al., 2015; Zilionis et al., 2017). After the quality control data filtering, we analyzed transcriptome of about 7000 single cells, with 200-7000 genes detected per cell and the mitochondrial gene ratio under 5\%. The mean reads per cell of two batches of independent samples were highly correlated (Figure 2figure supplement 1A), indicating negligible batch variance. According to cell type markers of the mice brain vessels identified by single-cell sequencing (He et al., 2018; Vanlandewijck et al., 2018), the cells in VOrs were clustered into 9 main cell types (Figure 2A), including fibroblast (FB), pericyte (PC), proliferative vascular progenitor $\left(\mathrm{MKI} 67^{+}\right.$ VP), endothelial cell (EC), smooth muscle cell (SMC), microglia (MG), immune cell (IM) and unknown cluster (Figure 2B). FB accounted for the highest proportion of total cells and MG the lowest (Figure 2-figure supplement 1B). We chose the top 5 highly expressed 
marker genes of each cluster (Figure 2-figure supplement 1C), and analyzed their expression patterns in each cell type (Figure 2C). Immunostaining showed that the vasculatures in VOrs exhibited positive signals of the SMC marker $\alpha \mathrm{SMA}$, the pericyte marker PDGFR $\beta$, and the EC marker CD31 (Figure 2D and E), confirming the results obtained using scRNA-seq. Meanwhile, the presence of MG-like cells was verified by the staining with specific markers TREM2 and TMEM119 (Figure 2F). Interestingly, DLL4 and EPHB4, which mark the venous and arteries endothelial cells, respectively (Vanlandewijck et al., 2018; Zhao et al., 2018), were found to express only in separate EC populations (Figure $2 \mathrm{G}$ and $\mathrm{H}$ ). This result indicates that ECs in VOrs already underwent spontaneous functional maturation. Immunostaining also confirmed the presence of the venous and arteries EC sub-types (Figure 2-figure supplement 1D and E). Thus, the formed VOrs contained the repertoire of brain-vessel cell types resembling that in vivo.

To depict the developmental process of VOrs, we reconstructed the time-course of vascular cell developmental trajectory in Pseudotime (Figure 3A and Figure 3-figure supplement 1A). Five developmental stages and two time points were showed in the trajectory, with stages 1 and 2 representing initial states, stage 3 representing the intermediate state, and stages 4 and 5 the latest (Figure 3-figure supplement $1 \mathrm{~A}$ and B). Then, we used a panel of markers to annotate the main cell types and found that FB and PC were among the early developed cell types while the EC and MG were among the later ones (Figure 3B-D and Figure 3-figure supplement 1C). It is known that PC and SMC constitute mural cells of blood vessels and it has been difficult to distinguish them because they have similar gene expression profiles (Smyth et al., 2018). Using the developmental trajectory analysis, we found that PC appeared earlier than SMC (Figure 3E). PC markers (MEF2C, PEGFR $\beta$, RGS5) were highly expressed in the early stages but down-regulated in the later stages, while SMC markers (ACTA2, MYL9, TAGLN) showed opposite tendency (Figure 3E). 
In order to determine to what extent the VOrs resembled the brain vessels in vivo, we analyzed two accessible datasets for comparison. First, we compared the VOrs and mouse cerebrovascular scRNA-seq data (He et al., 2018; Vanlandewijck et al., 2018), and found

188 that the molecular features of the five major cell types, including FB, SMC, PC, EC, and

189 MG, in VOrs were similar to the counterparts of mouse cerebrovascular system (Figure

190 3F). We then referred to a dataset of scRNA-seq from eight adult and four embryonic 191 human cortexes, which clustered a small number of vascular cell types, including EC, PC 192 and MG (Polioudakis et al., 2019). We analyzed the correlation of these three clusters in 193 VOrs, mouse and human samples together, and found that VOrs and human showed 194 stronger correlation in EC and PC clusters, while MG showed the highest consistency across all three datasets (Figure 3G). This result further confirmed the presence of brain

196 specific MG cells in VOrs culture system with the introduction of neurotrophic factors.

197 Thus, VOr is an appropriate model for the analysis of human cerebrovascular development 198 in vitro.

Next we analyzed differentially expressed genes (DEGs) in ECs between VOrs and mouse, human and mouse, respectively, and found that a big fraction of genes up-regulated were overlapped between the two sets of comparisons (Figure 3H). Remarkably, most of the top DEGs between VOrs and mouse groups showed similar tendency in human samples (Figure 3I), suggesting the high similarity of ECs in VOrs compared to that in human samples in vivo. The gene ontology (GO) analysis showed that the shared DEGs between VOr vs. mouse and human vs. mouse pairs were related to the angiogenesis pathway, 208 of mouse (Figure 3J). We also analyzed DEGs within PCs, and found that, the majority of 209 top changed genes in VOrs compared with mouse samples were also present in DEGs of 210 human vs. mouse pair comparison (Figure 3-figure supplement 1D and E). To further 211 validate that VOrs can faithfully mimic the process of vascular development in vivo, the 212 expression of marker genes of three major cell types from VOrs (EC, PC, MG) were 213 compared with that of human samples. As shown in Figure 3K, marker genes of VOrs were 
214 also highly expressed in the same cell types of human samples, further indicating the

215 similarity of corresponding cell type. Recently, Lu et al demonstrated that some in vitro

216 induced brain vessel cells lacked functional attributes of ECs but were more related to the

217 neuroectodermal epithelial lineage-induced brain microvascular endothelial cells (Epi-

218 iBMEC) (Lu et al., 2021). We performed principal componant analysis (PCA) for ECs in

219 VOrs and other 28 datasets from this study, including the primary ECs, induced ECs (iEC),

220 and Epi-iBMECs, and found that ECs in VOrs showed a clear disparity from Epi-iBMECs

221 but higher similarity to EC lineage (Figure 3-figure supplement 1F). The top and bottom

222 loading genes showed separate endothelial and epithelial cell type identities in these

223 datasets (Figure 3-figure supplement 1G), and VOrs exhibited strong endothelial cell

224 properties (Figure 3G). These results indicate that the VOr model can be used to

225 recapitulate cerebrovascular development in vitro.

227 Generation of fusion vascularized brain organoids

228 Having established the VOrs, we decided to generate vascularized brain organoids by using 229 co-culture strategy. For this purpose, we established the induction system of brain 230 organoids from human H9 ESCs according to the methods reported previously (Lancaster 231 and Knoblich, 2014; Mariani et al., 2012; Ou et al., 2020), with some modifications 232 (Figure4-figure supplement 1A). Cerebral organoids at different developmental stages 233 were stained with neural progenitor markers PAX6 and p-VIM, the proliferation marker 234 KI67, intermediate progenitor marker TBR2, young neuron marker DCX (doublecortin), 235 mature neuron marker TUJ1, and the cortical layer markers (TBR1, CTIP2, SATB2, 236 REELN), and the results indicated that the brain organoids were well induced (Figure 4237 figure supplement 1B-E). As expected, CD31 ${ }^{+}$ECs were not observed in this induction 238 system (Figure4-figure supplement 1F). After the step of neural ectoderm induction, EBs 239 with neuroepithelial (NE) property were co-embedded with VPs in one Matrigel droplet, 240 and then cultured under the condition of VOrs maturation with the medium containing N2 241 and B27 (Figure 4A). For better invasion of vessels into the developing BOrs, we put two 242 VP bodies in both sides of one NE body (Figure 4A). After co-culture for different days, 
243 VOrs labelled by GFP gradually wrapped BOrs and finally formed a fused vasculature and

244 brain organoids (fVBOrs) by D40 (Figure 4B). Whole-mount staining of the fVBOrs

245 showed that DCX-labelled neurons were enwrapped by invaded vessels labeled by CD31

246 (Figure 4C).

Supported by pericytes and astrocytes, the brain microvascular ECs form a particularly tight layer called the blood-brain-barrier (BBB), which selectively controls the flow of substances into and out of the brain by forming complex intercellular tight junctions and protects the brain from harmful substances (Augustin and Koh, 2017; Chow and Gu, 2015; Lippmann et al., 2012; Sweeney et al., 2019). To determine whether the fVBOrs developed BBB-like features, we examined the expression of the tight junctions proteins

254 Claudin5 (CLDN5) and ZO-1 (Figure 4D and Figure 4-figure supplement 2A and B), and 255 the efflux transporter p-Glycoprotein which helps the recycling of small lipophilic molecules diffused into ECs back to the blood stream (Augustin and Koh, 2017; Lippmann et al., 2012) (Figure4-figure supplement 2C). Notably, stronger CLDN5 signals were observed in BOr regions in contact with vessels, suggesting the appearance of tight junctions-like structures (Figure 4-figure supplement 2A). Furthermore, astrocyte-like cells labelled by S100 or GFAP were also observed in fusion organoids, forming neurovascular unit-like structures composed of CD31/GFP-labelled vascular structures and MAP2labelled neurons (Figure 4E). The presence of neurovascular unit structure in fusion organoids was also confirmed by Transmission Electronic Microscopy (TEM), which

264 showed the endothelial cell basement membrane enclosed by pericytes and tightly contacted by end-feet of astrocytes (Figure 4-figure supplement 2E).

Next, we examined the functionality of BBB in the fVBOrs, by measuring the permeability of molecules with different BBB penetration capability (Bergmann et al., 2018; Cho et al., 2017; Dai et al., 2018; Xu et al., 2019). The selectivity of BBB was

270 determined by incubating fVBOrs with rhodamine-labelled Angiopep-2, a peptide capable 271 of permeating through BBB selectively (Bergmann et al., 2018; Cho et al., 2017). We found 
272 that Angiopep-2 exhibited strong signals in the fVBOrs, but scrambled peptides displayed

273 no detectable signal (Figure 4F and G). The z-stack images showed that the intensity of

274 Angiopep-2 signals decreased from the surface to the inner of fVBOrs (Figure 4F, bottom).

275 In contrast to fusion organoids, the BOrs alone showed much weaker Angiopep-2 signals

276 (Figure 4-figure supplement 2E and F). Taken together, these results indicate that fVBOrs

277 have developed BBB structures with selective permeability.

\section{Microglia cells in fVBOrs are responsive to immune stimuli}

280 It is generally believed that MGs are developed from the yolk-sac progenitors, which then

281 populate in the developing brain to regulate neurogenesis and neural circuit refinement

282 (Kaur et al., 2017; Mosser et al., 2017; Salter and Stevens, 2017). Indeed, in the unguided

283 cerebral organoids, spontaneous MG can emerge (Ormel et al., 2018), probably due to the

284 presence of residue mesodermal progenitors (Quadrato et al., 2017). However, the

285 functional investigation is limited due to the variable and inconsistent batch effects. Based

286 on the scRNA-seq and staining results suggesting the presence of MG in VOrs, we decided

287 to explore the possibility of introducing these MG-like cells into brain organoids using

288 fusion strategy. To this end, we first determined the molecular features of MG in VOrs. As

289 shown in Figure 5A, the MG identity was confirmed by the expression of specific marker 290 genes AIF1 (the gene encoding IBA1) and CD68. The GO analysis showed that the 291 functions of MG markers were mainly concentrated on the pathways of immune and 292 inflammatory responses (Figure 5B). During the VOr culture, the expression of MG marker 293 genes, such as AIF1 or TMEM119, gradually increased (Figure 5C), indicating again the 294 MG induction. In line with this notion, D40 VOrs exhibited increased abundance of MG 295 with amoeboid-like morphology, compared to D25 MG mostly with round morphology 296 (Figure 5D and E).

We next examined whether MG could migrate from VOrs into BOrs after fusion. We found large amount of $\mathrm{IBA}^{+} \mathrm{GFP}^{+} \mathrm{MG}$-like cells in the neural part of fVBOrs, whereas BOrs alone had no MG-like signal (Figure 5F). Thus, fVBOrs also contained MG-like cells, 
301 besides vasculatures. Then, we determined the responsiveness of these MG-like cells to the 302 treatments used to diminish or activate microglia to verify the cell identity. First, we treated 303 D30 VOrs with PLX5622, a selective inhibitor of CSF1R (colony stimulating factor 1 304 receptor), which was used to ablate MG in mice (Huang et al., 2018). After treatment for 7 305 days with $2 \mu \mathrm{M}$ PLX5622, the IBA1-labelled cells were almost completely gone, and the 306 ablation effect lasted for at least 3 days in the absence of PLX5622 (Figure 5-figure 307 supplement 1A and B). Likewise, the PLX5622 treatment also depleted MG-like cells in 308 D40 fVBOrs (Figure 5-figure supplement 1C). These results further confirmed the identity 309 of MG-like cells in VOrs and fVBOrs. Next, fVBOrs were treated with $0.5 \mu \mathrm{g} / \mathrm{ml}$ 310 lipopolysaccharide (LPS) for $72 \mathrm{~h}$ to induce inflammatory response. The LPS stimulation 311 caused marked increase in the expression of inflammatory factors TNF $\alpha$ and IL-6 (Figure 312 5G). Interestingly, the expression levels of TNF $\alpha$ or IL-6 were attenuated in PLX5622313 treated fusion organoids (Figure 5G), suggesting the involvement of MG-like cells in LPS314 induced immune response. These results support the conclusion that MG-like cells possess 315 responsive ability to immune stimuli.

\section{Increased neural progenitors in the fusion organoids}

318 It has been shown that vasculature acts as a critical niche that helps maintain the survival 319 and stemness of neural progenitors, and EC-derived soluble factors might contribute to this 320 function (Delgado et al., 2014; Ottone et al., 2014; Shen et al., 2004). Prompted by these 321 information, we compared neurogenesis patterns in fVBOr and BOr. Interestingly, the 322 fVBOrs exhibited marked increase in the thickness of neuroepithelial rosettes compared to 323 BOrs at the same corresponding stages (D25) (Figure 6A and B). In line with this notion, 324 the density of neural progenitors (NP) marked by PAX6 or mitotic NPs marked by 325 phospho-vimentin (P-VIM) also increased in fusion organoids (Figure 6C-E). However, 326 the density of DCX-labelled differentiated neurons or TBR1-labelled early born cortical 327 neurons had no difference at the observation period (Figure 6F and G; Figure 6-figure 328 supplement $1 \mathrm{~A}$ and B). These results suggest that the factors produced by VOrs might 329 promote the proliferation of NPs after fusion with BOrs, with little effect on neuronal 
330 differentiation. In the classical brain organoid culture system, the inner cells are extremely 331 vulnerable to limited accessibility to the trophic factors in culture medium. In line with this 332 notion, BOrs at D40 showed abundant apoptotic cells expressing cleaved caspase 3 (c333 CASP3) in the central regions, whereas the apoptotic cells were markedly reduced in 334 fVBOrs (Figure 6-figure supplement 1C-E). Thus, the fusion organoids generated in this work can be used to study interactions among multiple cell types during brain development.

Discussion

338 The prevalent approaches for brain organoid induction start from neuroectoderm induction 339 using BMP and/or Wnt signaling inhibitors, or neural induction medium to treat embryoid 340 bodies, followed by brain regional differentiation, and neural maturation (Giandomenico 341 and Lancaster, 2017; Kadoshima et al., 2013; Lancaster and Knoblich, 2014; Lancaster et 342 al., 2013; Qian et al., 2016). Although this system is powerful, it lacks vascularization 343 rendering it impossible to recapitulate vascular-neural interactions and model related 344 diseases. Here, we developed an approach for generating neural-specific vessel organoids 345 by initial transient mesoderm induction, sequential VP and EC induction, followed by 346 treatments with neurotrophic reagents. Then, we established an integrated vascularized 347 brain organoid model by fusing the brain and vessel organoids. This vascularization 348 strategy considered the compatibility to mesodermal and ectodermal linages and led to 349 generation of brain organoids with complex tubular vessels, functional neurovasculature 350 units, as well as microglia responding to immune stimulation.

The fVBOr model generated in this study provides a possibility to analyze the process 353 of brain angiogenesis and complex interactions between vasculatures and neural cells. It 354 has been shown previously that brain vascularization is regulated by neural progenitors 355 (Matsuoka et al., 2017), and, on the other hand, vasculatures promote neurogenesis and 356 oligodendrocyte precursor migration (Tata et al., 2016; Tsai et al., 2016). Notably, we 357 found that in fVBOrs, only vasculatures close to or located in the BOrs expressed tight 
358

359

junctions markers, including CLDN5 and ZO-1 (Figure 4-figure supplement 2A and B), consistent with the idea that BBB maturation is regulated by neural cues (Lippmann et al., 2012). The effects of vasculatures on neurogenesis were also found in fVBOrs, which exhibited increased pool of neural progenitors and reduced apoptosis (Figure 6C-E; Figure 6-figure supplement 1C-E). The reduction in apoptotic cells was also seen in grafted BOrs with the invasion of host blood vessels (Mansour et al., 2018; Shi et al., 2020). It is conceivable that the vascularized brain organoids developed in this study may provide a feasible platform for the study of human brain development, vasculature-related diseases or pharmaceutical interventions, which need to pass the BBB barrier.

Several studies have tried to generate BBB-like structures in vitro, by culturing PSCderived ECs (Lippmann et al., 2012; Qian et al., 2017) or co-culturing PSC-derived cells without or with primary cells in 2D system (Appelt-Menzel et al., 2017; Canfield et al., 2017) or 3D system (Bergmann et al., 2018; Cho et al., 2017). Although some features of the $\mathrm{BBB}$ were reproduced in these studies, the tube-like structures of blood vessels were lacking. The vessel organoid model we have established showed intense vascular network with characteristics and specificity of human cerebral vessels based on transcriptomic analysis and functional assay. It has been demonstrated that the combination of PSCderived tissue-specific progenitors or relevant tissue samples with ECs and mesenchymal stem cells can generate vascularized organs (Takebe et al., 2015; Takebe et al., 2013), but different derivation of vascular ECs and the brain from distinct germ layers limits the vascularization in BOrs initialized from neuroectoderm induction. Indeed, we failed to observe any vascular structures in the brain organoids generated using the prevalent approach (Figure 4-figure supplement 1F). Although the fVBOr model has shown branched vessels, it still lacks active blood flow. One possible approach to solve the problem is to combine microfluidic techniques and organoid cultures and make an "organon-a-chip", which may authentically mimic the vascular environment. 
The blood vessels in the brain are not only required for oxygen and nutrient supply, but also involved in regulating neurogenesis. The fusion organoids allow for recapitulating early developmental processes like vasculogenesis and angiogenesis, as well as the integration of microglia. Unlike BBB-like structures generated in other studies that combined various mature cell types (Cho et al., 2017; Lippmann et al., 2012; Qian et al., 2017), the ones generated in our study were directly induced from pluripotent stem cells,

392 which resembled the developmental processes in vivo. Although the factors that defined

393 the identity of microglia cells in VOrs are not clear, their migration into the brain organoids

394 resembled the extra-embryonic originality. It is known that astrocytes are essential 395 components of the neurovascular units (Abbott et al., 2006), their involvement in the maturation of BBB-like units or immune surveillance awaits for further investigation.

\section{Materials and Methods}

\section{hESCs culture}

400 H9 human embryonic stem (H9-hES) cell line was purchased from iMedCell. H9-hES401 EGFP was generated by introducing the CAG-EGFP DNA fragment into the genome locus 402 ROSAßgeo26 (ROSA26) using the CRISPR/Cas9 method. Both H9-hES and H9-hES403 EGFP cells were cultured and passaged as previously described (Ou et al., 2020). Cells 404 were cultured on hESC-Matrigel (Corning) coated dishes in mTeSR1 (STEMCELL) 405 medium with the addition of bFGF $(4 \mu \mathrm{g} / \mathrm{ml}$, STEMCELL). The culture medium was half406 replaced every day and then cells were passaged every 5 days using passage reagent 407 ReLeSR (STEMCELL).

\section{Generation of human brain organoid}

$410 h$ ESC clones were dissociated into single cells with Accutase (STEMCELL), then cells 411 were re-suspended in mTeSR1 medium containing $10 \mu \mathrm{M}$ Y27632 (STEMCELL), and 412 seeded into the lipidure-coated (NOF CORPRATION) V-bottom 96-well plate (Thermo) 413 with 7000 cells per aggregate, $150 \mu \mathrm{l}$ per well to form EBs. On day 2, the culture medium 414 was replaced by the ectodermal induction medium (DMEM/F12 (Life/Invitrogen) 
415 containing 20\% (v/v) Knockout Serum Replacer (Gibco), 1\% (v/v) MEM-NEAA (Gibco),

$4163.5 \mu \mathrm{l} / 1 \quad \beta$-mercaptoethanol (Sigma-Aldrich), 1\% (v/v) Glutamax (Gibco), $2.5 \mu \mathrm{M}$

417 dorsomorphine (Tocris) and $2 \mu \mathrm{M}$ A83-01 (Tocris)). On day 4, the ectodermal induction

418 medium was half-replaced. On day 6, the EB medium was replaced by the neural induction

419 medium (DMEM/F12 containing 1\% (v/v) N2 supplement (Life/Invitrogen), 1\% (v/v)

420 MEM-NEAA, $1 \%$ (v/v) Glutamax, $1 \mu \mathrm{g} / \mathrm{ml}$ heparin (Sigma-Aldrich,), $10 \mu \mathrm{M}$ SB431542

421 (Selleck) and 200 nM LDN193189 2HCL (Selleck)) and lasted for 6 days. The neural

422 induction medium was half-renewed every other day. On day 12, the EBs were embedded

423 into growth factor-reduced Matrigel droplet (Corning), as described previously (Lancaster

424 and Knoblich, 2014). And the culture medium was replaced by the differentiation medium

$425(50 \%(\mathrm{v} / \mathrm{v})$ DMEM/F12 and 50\% (v/v) Neurobasal medium (Life/Invitrogen) containing

$426 \quad 0.5 \% \quad(\mathrm{v} / \mathrm{v}) \quad \mathrm{N} 2$ supplement, $0.5 \% \quad(\mathrm{v} / \mathrm{v}) \quad \mathrm{B} 27$ supplement without vitamin A

427 (Life/Invitrogen), $3.5 \mu \mathrm{l} / 1 \beta$-mercaptoethanol, $250 \mu \mathrm{l} / 1$ Insulin (Sigma-Aldrich), 1\% (v/v)

428 Glutamax and 0.5\% (v/v) MEM-NEAA, 1\% (v/v) Antibiotic-Antimycotic (Gibco)). After

4294 days, the differentiation medium was replaced by the maturation medium (50\% (v/v)

$430 \mathrm{DMEM} / \mathrm{F} 12$ and 50\% (v/v) Neurobasal medium containing 0.5\% (v/v) N2 supplement,

$4310.5 \%$ (v/v) B27 supplement (Life/Invitrogen), $3.5 \mu 1 / 1 \beta$-mercaptoethanol, $250 \mu 1 / 1$ Insulin,

$4321 \%(\mathrm{v} / \mathrm{v})$ Glutamax and 0.5\% (v/v) MEM-NEAA, $1 \%(\mathrm{v} / \mathrm{v})$ Antibiotic-Antimycotic). Then,

433 the organoids were transferred into a shaker in the $5 \% \mathrm{CO}_{2}$ incubator at $37^{\circ} \mathrm{C}$ for

434 maturation, and the medium was renewed every 3-4 days.

\section{Generation of human vessel organoid}

437 H9-hES-GFP clones were dissociated into single cells with Accutase, then the cells were 438 re-suspended in the mTeSR1 medium containing $10 \mu \mathrm{M}$ Y27632 and seeded into the 439 lipidure-coated V-bottom 96-well plate with 9000 cells per aggregate, $150 \mu$ per well to 440 form EBs. On day 2, the culture medium was replaced by the mesodermal induction 441 medium (APEL2 (STEMCELL) with $6 \mu \mathrm{M}$ CHIR99021 (Selleck)). On day 4, the 442 mesodermal medium was replaced by endothelial induction medium (APEL2 with 50 $443 \mu \mathrm{g} / \mathrm{ml}$ VEGF (STEMCELL), $25 \mu \mathrm{g} / \mathrm{ml}$ BMP4 (R\&D, 314-BP) and $10 \mu \mathrm{g} / \mathrm{ml} \mathrm{bFGF}$ ). On 
444 day 7, medium was changed into MV2 medium (Promocell) with $50 \mu \mathrm{g} / \mathrm{ml}$ VEGF for the

445 maturation of endothelial cells, and the medium was renewed every other day. From day

446 12, the EBs were embedded into Matrigel droplets and cultured with VEGF-containing (20

$447 \mu \mathrm{g} / \mathrm{ml})$ neural differentiation medium, as that used for brain organoid culture.

449 Fusion of vascular brain organoids

450 To generate the fusion organoids, two VOr EBs and one BOr EB were collected and then 451 embedded together into one Matrigel droplet $(25 \mu \mathrm{l})$ on day 12. The two VOr EBs were put 452 on two sides of the BOr EB, and pipette tips could be used to adjust the shape and site of 453 the three EBs. The following steps were the same as the non-fusion BOr EBs with the 454 addition of $20 \mu \mathrm{g} / \mathrm{ml}$ VEGF.

\section{Immunofluorescence}

457 The collected organoid samples were fixed in $4 \%$ paraformaldehyde (PFA) at $4{ }^{\circ} \mathrm{C}$ 458 overnight, and then washed 3 times with PBS, dehydrated in $30 \%$ sucrose at $4^{\circ} \mathrm{C}$ for $24-48$

459 hr. Then, organoids were embedded in O.C.T (Sakura) and cryosectioned into $30 \mu \mathrm{m}$-thick 460 slices. The sectioned slices were boiled in citrate-based antigen retrieval buffer for $10 \mathrm{~min}$, 461 followed by cooling for over $60 \mathrm{~min}$. Slices were washed in PBS for 3 times and incubated 462 in $0.3 \%$ TritonX-100 (Sigma-Aldrich,) at room temperature (RT) for $30 \mathrm{~min}$, blocked with $4635 \%$ BSA (Sigma-Aldrich) in $0.1 \%$ TritonX-100 at RT for $1 \mathrm{hr}$, incubated with the primary 464 antibody at $4{ }^{\circ} \mathrm{C}$ for over $48 \mathrm{hr}$, followed by washes with PBS and incubation with the 465 secondary antibody at $4^{\circ} \mathrm{C}$ overnight. Secondary antibodies were: AlexaFluor 488,555 , 466 594, or 647-conjugated donkey anti-mouse, -rabbit, -rat or -chicken IgG (Invitrogen, all 467 used at 1:1000 dilution). DAPI (beyotime, 1:2000 dilution) was used to mark cell nuclei. 468 Stained sections were mounted with mounting medium and stored at $4^{\circ} \mathrm{C}$ before imaging. 469 All images were acquired by confocal imaging systems.

471 For whole-mount staining, the organoid samples were fixed in $4 \%$ PFA at $4{ }^{\circ} \mathrm{C}$ 472 overnight, and then washed for 3 times with PBS, followed by the incubation in $0.5 \%$ 
473 TritonX-100 at RT for $1 \mathrm{hr}$. After blocking with 5\% BSA in 0.1\% TritonX-100 at RT for

$4741 \mathrm{hr}$, organoids were incubated with primary antibodies at $4^{\circ} \mathrm{C}$ for over $48 \mathrm{hr}$, washed with

475 PBS, and then incubated with secondary antibodies at $4^{\circ} \mathrm{C}$ for over $48 \mathrm{hr}$. The stained

476 organoids were washed by PBS for 3 times before confocal imaging.

478 Quantitative PCR (qPCR)

479 The total RNA of 3-4 organoids was extracted using the RNeasy Plus Micro Kit (Qiagen),

480 followed by reverse transcription to generate cDNA with GoScript Reverse Transcription

481 Kit (Promega). Quantitative PCR was performed by using the Agilent Mx3000P qPCR

482 system with the $2 \times$ SYBR Green qPCR Master Mix (Bimake). Relative mRNA expression

483 was determined by the delta cycle time with human GAPDH as the internal control in data

484 normalization. Primer sequences were as follows:

485 TNF- $\alpha$ : forward, 5' - CACAGTGAAGTGCTGGCAAC -3', reverse, 5'-

486 AGGAAGGCCTAAGGTCCACT -3';

487 IL-6: forward, 5' - TTCCAAAGATGTAGCCGCCC-3', reverse, 5'-

488 ACCAGGCAAGTCTCCTCATT-3';

489 GAPDH: forward, 5'-TCGGAGTCAACGGATTTGGT-3', reverse, 5'-

490 TTCCCGTTCTCAGCCTTGAC-3';

491 IBA1: forward, 5'-AAACCAGGGATTTACAGGGAGG-3', reverse, 5'-

492 GGGCAGATCCTCATCACTGC-3';

493 TMEM119: forward, 5'-GAGGAGGGACGGGAGGAG-3', reverse: 5'-

494 GACCAGTTCCTTGGCGTACA-3’;

495 NANOG: forward, 5'-CAATGGTGTGACGCAGAAGG-3', reverse: 5'-

496 TGCACCAGGTCTGAGTGTTC-3';

497 OCT4: forward, 5'-CTCGAGAAGGATGTGGTCCG-3', reverse, 5'-

498 TGACGGAGACAGGGGGAAAG-3';

499 PECAM1: forward, 5'-AGACGTGCAGTACACGGAAG-3', reverse, 5' -

500 TTTCCACGGCATCAGGGAC-3'; 
501 VE-Cadherin: forward, 5'-CGCAATAGACAAGGACATAACAC-3', reverse, 5'-

502 GGTCAAACTGCCCATACTTG-3’;

503 VWF: forward, 5'-CCCGAAAGGCCAGGTGTA-3', reverse, 5'-

504 AGCAAGCTTCCGGGGACT-3’; VEGFR2: forward, 5’-

505 GAGGGGAACTGAAGACAGGC-3', reverse, 5'-GGCCAAGAGGCTTACCTAGC-3';

506 VEGFR1: forward, 5'-AACGTGGTTAACCTGCTGGG-3', reverse, 5'-

507 AGTGCTGCATCCTTGTTGAGA-3’;

508 PDGFR: forward, 5'-ATCAGCAGCAAGGCGAGC-3', reverse, 5'-

509 CAGGTCAGAACGAAGGTGCT-3’.

511 Flow cytometry

512 VOrs were dissociated with Trypsin solution as described in single-cell dissociation. After

513 re-suspension in staining buffer, about $1 \times 10^{6}$ single cells for each group were incubated

514 with Alexa 647-labeled CD31 antibody (1:1000 dilution, BD) for $30 \mathrm{~min}$. The results were

515 analyzed by using FlowJo software. Single cells isolated from BOrs were used as the

516 negative control.

\section{$518 \quad$ LDL-uptake assay}

519 hESC (D0) and VOrs (D4-D40) were washed with PBS for 3 times and then incubated with

$52010 \mu \mathrm{g} / \mathrm{ml} \mathrm{Ac-LDL}$ (Yeasen) in MV2 medium for $4 \mathrm{hr}$ at $37^{\circ} \mathrm{C}$. The samples were washed

521 for 3 times with PBS, before confocal imaging using 20xobjective lens.

\section{BBB penetrating assay}

524 fVBOrs were collected in 35-mm dishes and washed with PBS for 3 times, followed by 525 incubation with $5 \mu \mathrm{M}$ angiopep (TAMRA-TFFYGGSRGKRNNFKTEEY) or control 526 scrambled (TAMRA-GNYTSRFEREYGKFNKFGT) peptides in neuronal maturation 527 medium for $3 \mathrm{hr}$ at $37^{\circ} \mathrm{C}$. Then, the organoids were washed with PBS, fixed in $4 \%$ PFA 528 containing DAPI to stain the cell nuclei, and then imaged using $1.25 \times$ objective lens. 


\section{Transmission Electron Microscope (TEM) analysis}

531 Cultured fVBOrs were washed with DPBS (Life/Invitrogen) and cut into $1 \mathrm{~mm} \times 1 \mathrm{~mm}$

532 small pieces. Firstly samples were fixed with $4 \%$ paraformaldehyde overnight and then

533 were pre-fixed with $2.5 \%$ glutaraldehyde (SPI, USA), in PBS for $12 \mathrm{hr}$. After washing with

534 PBS, samples were post-fixed with $1 \%$ OsO4 (TED PELLA) for $2 \mathrm{hr}$ at $4^{\circ} \mathrm{C}$. Next, followed

535 by dehydrated in an ascending gradual series (30\%-100\% (v/v)) of ethanol, and embedded

536 in epoxy resin (Pon812 kit, SPI, USA). The embedded samples were initially cut into about

$537500 \mathrm{~nm}$-thick sections, inspected by stained with toluidine blue (Sinopharm), and finally

538 sectioned into 70-nm by Leica EM UC7. Then sections were double-stained with uranyl

539 acetate (SPI, USA) and lead citrate (SPI, USA), followed by observation with a

540 Transmission Electron Microscopy (Talos L120C) at an acceleration voltage of $80 \mathrm{kV}$.

\section{Single cell dissociation and 10x genomics chromium library construction}

543 Organoids were dissociated using the methods as described previously (Thomsen et al.,

544 2016). Briefly, 8-10 organoids were collected and washed by DPBS (Life/Invitrogen,) and

545 cut into small pieces, followed by the incubation with $2 \mathrm{~mL}$ trypsin solution $\left(\mathrm{Ca}^{2+} / \mathrm{Mg}^{2+}\right.$ -

546 free HBSS (Life/Invitrogen) with $10 \mathrm{mM}$ HEPES (Sigma-Aldrich), $2 \mathrm{mM} \mathrm{MgCl} 2,10 \mu \mathrm{g} / \mathrm{ml}$

547 DNase I (Roche), $0.25 \mathrm{mg} / \mathrm{ml}$ trypsin (Sigma-Aldrich), $\mathrm{PH} 7.6$ ) for $30 \mathrm{~min}$ at $37^{\circ} \mathrm{C}$. Then

548 the samples were quenched with $4 \mathrm{ml}$ Quenching Buffer (440 ml Leibovitz L-15 medium

549 (Thermo) with $50 \mathrm{ml} \mathrm{ddH}{ }_{2} \mathrm{O}, 5 \mathrm{ml} 1$ M HEPES (pH 7.3-7.4), $10 \mu \mathrm{g} / \mathrm{ml}$ DNase I, $100 \mathrm{nM}$

550 TTX (TOCRIS), $20 \mu \mathrm{M}$ DNQX (TOCRIS), and $50 \mu \mathrm{M}$ DL-AP5 (TOCRIS), $5 \mathrm{ml}$ 100×

551 Anti-Anti, $2 \mathrm{mg} / \mathrm{ml} \mathrm{BSA,} 100 \mu \mathrm{g} / \mathrm{ml}$ trypsin inhibitor (Sigma-Aldrich)), subjected to

552 centrifugation in $220 \times \mathrm{g}$ for $4 \mathrm{~min}$ at $4^{\circ} \mathrm{C}$, and re-suspended with $2 \mathrm{ml}$ Staining Medium

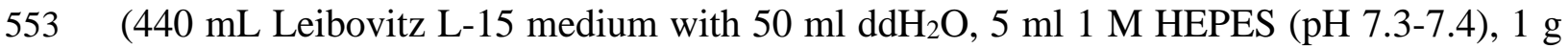

554 BSA, $100 \mathrm{nM}$ TTX, $20 \mu \mathrm{M}$ DNQX, and $50 \mu \mathrm{M}$ DL-AP5, $5 \mathrm{ml}$ 100× Anti-Anti, $20 \mathrm{ml} 77.7$

555 mM EDTA (pH 8.0)), filtered through a 40 micron cell filter (Falcon), centrifuged again in

$556220 \times \mathrm{g}$ for $4 \mathrm{~min}$ at $4{ }^{\circ} \mathrm{C}$, then the cells were re-suspended in $5 \mathrm{ml}$ DPBS with $1 \%$ BSA.

557 Dissociated cells were re-suspended at a concentration of 500 cells/ $\mu 1$. cDNA libraries were 558 generated following the guidelines provided by 10x Genomics, Inc. Briefly, dissociated 
559 cells were partitioned into nanoliter-scale Gel Bead-In-Emulsions (GEMs), and then

560 subjected to reverse transcription, cDNA amplification and library construction, with

561 individual cell and transcript barcoded.

562

\section{Single-cell RNA-Seq data analysis}

564 Cellranger software was used for mapping raw data to the human genome (version hg38 565 (v1.2.0)). And then data were processed with Seurat (v3.0) under R (v3.5.2) environment.

566 For quality control, cells expressing less than 200 genes or more than 7000 genes were 567 removed, and genes expressed in less than 3 cells were excluded for the following analysis.

568 VST (variance-stabilizing transformation) was used for searching for the highly-variable 569 genes and the top 2,000 genes were chosen to do the downstream analysis. The cells were 570 clustered and reduced into the UMAP space by principal component analysis (PCA), with 571 1st to 15 th principal components (PCs). Differentially expressed genes (DEGs) in each 572 cluster were identified by more than 1.25 fold change and $p$ value $<0.05$ with Wilcoxon 573 Rank Sum test. GO enrichment analysis was carried out by 'ClusterProfiler' and 574 org.Hs.eg.db in R software program with $\mathrm{p}$ value $<0.1$ and FDR (false discovery rate) < $575 \quad 0.05$ considered as statistical significance.

Monocle (v2.10.1) package was used to analyze the developmental trajectory of cell 578 types. The monocle object was constructed from the Seurat object. After normalization and 579 variance estimation, we calculated the mean and dispersion values and chose the genes 580 whose mean expression were $>0.1$. Then cells were dimensional reduced and clustered. 581 DEGs with $>1.25$ fold change and $\mathrm{p}$ value $<0.05$ (two-side $\mathrm{t}$ test) were used for downstream 582 analysis. The cell trajectory plots were produced by running 'reduceDimension' and 583 'orderCell' function with defined option.

Human neocortical scRNA-seq data (Polioudakis et al., 2019) (phs001836) and mouse

586 brain vascular scRNA-seq data (He et al., 2018; Vanlandewijck et al., 2018) (GSE98816) 587 were downloaded for the correlation analysis. The pearson correlation coefficient 
calculated by the intersect genes between different data sets was used for correlation analysis. DEGs were set with 1.25 fold change and p value $<0.05$ by limma (v3.38.3) package.

591

592

593

Data availability

Single cell RNA sequencing transcriptome data supporting this study have been deposited in NCBI Sequence Read Archive (SRA) repository (https://www.ncbi.nlm.nih.gov/sra) with accession number SRP338043 (VOR: SRR15992286; VOR2:SRR15992285). All

\section{Acknowledgments}

599 This study was partially supported by the National Key Research and Development 600 Program of China (2021ZD0202500), National Natural Science Foundation of China 601 (32130035 and 92168107 to Z.G.L, 31871034 to X.C.J), the Frontier Key Project of the 602 Chinese Academy of Sciences (QYZDJ-SSW-SMC025), Shanghai Municipal Science and 603 Technology Projects (2018SHZDZX05, 201409001700), and National Key R\&D Program 604 of China (2017YFA0700500). We are grateful to the Multi-Omics Core Facility, Molecular 605 Imaging Core Facility and Molecular and Cell Biology Core Facility at the School of Life 606 Science and Technology, ShanghaiTech University for providing technical support.

\section{Author Contributions}

609 X.Y.S., X.C.J., designed and conducted the experiments; Y.J.C contributed unpublished 610 reagents; X.Y.S., X.C.J., Y.L., P.M.Z., performed the experiments; X.Y.S., P.M.Z., L.B.S., 611 performed the single-cell analysis; Y.L., J.W. participated in sample preparation; X.Y.S 612 and Z.G.L wrote the paper. Z.G.L supervised the whole work.

\section{Declaration of Interests}

615 The authors declare no competing interests. 
618

619

\section{Reference}

Abbott NJ, Ronnback L, and Hansson E. 2006. Astrocyte-endothelial interactions at the blood-brain barrier. Nat Rev Neurosci 7: 41-53. doi: http://dx.doi.org/10.1038/nrn1824

Amin ND, and Pasca SP. 2018. Building Models of Brain Disorders with Three-Dimensional Organoids. Neuron 100: 389-405. doi: http://dx.doi.org/10.1016/j.neuron.2018.10.007

Appelt-Menzel A, Cubukova A, Gunther K, Edenhofer F, Piontek J, Krause G, Stuber T, Walles H, Neuhaus W, and Metzger M. 2017. Establishment of a Human Blood-Brain Barrier Co-culture Model Mimicking the Neurovascular Unit Using Induced Pluri- and Multipotent Stem Cells. Stem Cell Reports 8: 894-906. doi: http://dx.doi.org/10.1016/j.stemcr.2017.02.021

Augustin HG, and Koh GY. 2017. Organotypic vasculature: From descriptive heterogeneity to functional pathophysiology. Science 357. doi: http://dx.doi.org/10.1126/science.aal2379

Augustin HG, and Koh GY. 2017. Organotypic vasculature: From descriptive heterogeneity to functional pathophysiology. Science 357: eaal2379. doi: http://dx.doi.org/10.1126/science.aal2379

Bergmann S, Lawler SE, Qu Y, Fadzen CM, Wolfe JM, Regan MS, Pentelute BL, Agar NYR, and Cho CF. 2018. Blood-brain-barrier organoids for investigating the permeability of CNS therapeutics. Nat Protoc 13: 2827-2843. doi: http://dx.doi.org/10.1038/s41596-018-0066-x

Cai J, Pardali E, Sanchez-Duffhues G, and ten Dijke P. 2012. BMP signaling in vascular diseases. FEBS Lett 586: 1993-2002. doi: http://dx.doi.org/10.1016/j.febslet.2012.04.030

Cakir B, Xiang Y, Tanaka Y, Kural MH, Parent M, Kang YJ, Chapeton K, Patterson B, Yuan Y, He CS, et al. 2019. Engineering of human brain organoids with a functional vascular-like system. Nat Methods 16: 1169-1175. doi: http://dx.doi.org/10.1038/s41592-019-0586-5

Canfield SG, Stebbins MJ, Morales BS, Asai SW, Vatine GD, Svendsen CN, Palecek SP, and Shusta EV. 2017. An isogenic blood-brain barrier model comprising brain endothelial cells, astrocytes, and neurons derived from human induced pluripotent stem cells. $J$ Neurochem 140: 874-888. doi: http://dx.doi.org/10.1111/jnc.13923

Cho CF, Wolfe JM, Fadzen CM, Calligaris D, Hornburg K, Chiocca EA, Agar NYR, Pentelute BL, and Lawler SE. 2017. Blood-brain-barrier spheroids as an in vitro screening platform for brain-penetrating agents. Nat Commun 8: 15623. doi: http://dx.doi.org/10.1038/ncomms15623

Chow BW, and Gu C. 2015. The molecular constituents of the blood-brain barrier. Trends Neurosci 38: 598608. doi: http://dx.doi.org/10.1016/j.tins.2015.08.003

Cleaver O, and Melton DA. 2003. Endothelial signaling during development. Nat Med 9: 661-668. doi: http://dx.doi.org/10.1038/nm0603-661

Dai T, Jiang K, and Lu W. 2018. Liposomes and lipid disks traverse the BBB and BBTB as intact forms as revealed by two-step Forster resonance energy transfer imaging. Acta Pharm Sin B 8: 261-271. doi: http://dx.doi.org/10.1016/j.apsb.2018.01.004

Delgado AC, Ferron SR, Vicente D, Porlan E, Perez-Villalba A, Trujillo CM, D'Ocon P, and Farinas I. 2014. Endothelial NT-3 delivered by vasculature and CSF promotes quiescence of subependymal neural stem cells through nitric oxide induction. Neuron 83: 572-585. doi: http://dx.doi.org/10.1016/j.neuron.2014.06.015 Di Lullo E, and Kriegstein AR. 2017. The use of brain organoids to investigate neural development and disease. Nat Rev Neurosci 18: 573-584. doi: http://dx.doi.org/10.1038/nrn.2017.107

Giandomenico SL, and Lancaster MA. 2017. Probing human brain evolution and development in organoids. Curr Opin Cell Biol 44: 36-43. doi: http://dx.doi.org/10.1016/j.ceb.2017.01.001 
660 Gupta S, Zhu H, Zon LI, and Evans T. 2006. BMP signaling restricts hemato-vascular development from
661 lateral mesoderm during somitogenesis. Development 133: 2177-2187. doi:

661

662

663

664

665

666

667

668

669

670

671

672

673

674

675

676

677

678

679

680

681

682

683

684

685

686

687

688

689

690

691

692

693

694

695

696

697

698

699

700

701

702 http://dx.doi.org/10.1242/dev.02386

Harding A, Cortez-Toledo E, Magner NL, Beegle JR, Coleal-Bergum DP, Hao D, Wang A, Nolta JA, and Zhou P. 2017. Highly Efficient Differentiation of Endothelial Cells from Pluripotent Stem Cells Requires the MAPK and the PI3K Pathways. Stem Cells 35: 909-919. doi: http://dx.doi.org/10.1002/stem.2577

He L, Vanlandewijck M, Mae MA, Andrae J, Ando K, Del Gaudio F, Nahar K, Lebouvier T, Lavina B, Gouveia L, et al. 2018. Single-cell RNA sequencing of mouse brain and lung vascular and vessel-associated cell types. Sci Data 5: 180160. doi: http://dx.doi.org/10.1038/sdata.2018.160

Huang Y, Xu Z, Xiong S, Sun F, Qin G, Hu G, Wang J, Zhao L, Liang YX, Wu T, et al. 2018. Repopulated microglia are solely derived from the proliferation of residual microglia after acute depletion. Nat Neurosci 21: 530-540. doi: http://dx.doi.org/10.1038/s41593-018-0090-8

Jih YJ, Lien WH, Tsai WC, Yang GW, Li C, and Wu LW. 2001. Distinct regulation of genes by bFGF and VEGF-A in endothelial cells. Angiogenesis 4: 313-321. doi: http://dx.doi.org/10.1023/a:1016080321956

Kadoshima T, Sakaguchi H, Nakano T, Soen M, Ando S, Eiraku M, and Sasai Y. 2013. Self-organization of axial polarity, inside-out layer pattern, and species-specific progenitor dynamics in human ES cell-derived neocortex. Proc Natl Acad Sci USA 110: 20284-20289. doi: http://dx.doi.org/10.1073/pnas.1315710110

Kaur C, Rathnasamy G, and Ling EA. 2017. Biology of Microglia in the Developing Brain. J Neuropathol Exp Neurol 76: 736-753. doi: http://dx.doi.org/10.1093/jnen/nlx056

Kelava I, and Lancaster MA. 2016. Dishing out mini-brains: Current progress and future prospects in brain organoid research. Dev Biol 420: 199-209. doi: http://dx.doi.org/10.1016/j.ydbio.2016.06.037

Kelava I, and Lancaster MA. 2016. Stem Cell Models of Human Brain Development. Cell Stem Cell 18: 736748. doi: http://dx.doi.org/10.1016/j.stem.2016.05.022

Lancaster MA, and Knoblich JA. 2014. Generation of cerebral organoids from human pluripotent stem cells. Nat Protoc 9: 2329-2340. doi: http://dx.doi.org/10.1038/nprot.2014.158

Lancaster MA, Renner M, Martin CA, Wenzel D, Bicknell LS, Hurles ME, Homfray T, Penninger JM, Jackson AP, and Knoblich JA. 2013. Cerebral organoids model human brain development and microcephaly. Nature 501: 373-379. doi: http://dx.doi.org/10.1038/nature12517

Lee HS, Han J, Bai HJ, and Kim KW. 2009. Brain angiogenesis in developmental and pathological processes: regulation, molecular and cellular communication at the neurovascular interface. FEBS $J$ 276: 4622-4635. doi: http://dx.doi.org/10.1111/j.1742-4658.2009.07174.x

Lehle K, Friedl L, Wilm J, Philipp A, Muller T, Lubnow M, and Schmid C. 2016. Accumulation of Multipotent Progenitor Cells on Polymethylpentene Membranes During Extracorporeal Membrane Oxygenation. Artif Organs 40: 577-585. doi: http://dx.doi.org/10.1111/aor.12599

Lindsley RC, Gill JG, Kyba M, Murphy TL, and Murphy KM. 2006. Canonical Wnt signaling is required for development of embryonic stem cell-derived mesoderm. Development 133: 3787-3796. doi: http://dx.doi.org/10.1242/dev.02551

Lippmann ES, Azarin SM, Kay JE, Nessler RA, Wilson HK, Al-Ahmad A, Palecek SP, and Shusta EV. 2012. Derivation of blood-brain barrier endothelial cells from human pluripotent stem cells. Nat Biotechnol 30: 783-791. doi: http://dx.doi.org/10.1038/nbt.2247

Lopez-Ramirez MA, Pham A, Girard R, Wyseure T, Hale P, Yamashita A, Koskimaki J, Polster S, Saadat L, Romero IA, et al. 2019. Cerebral cavernous malformations form an anticoagulant vascular domain in humans and mice. Blood 133: 193-204. doi: http://dx.doi.org/10.1182/blood-2018-06-856062 
Lu TM, Houghton S, Magdeldin T, Duran JGB, Minotti AP, Snead A, Sproul A, Nguyen DT, Xiang J, Fine HA, et al. 2021. Pluripotent stem cell-derived epithelium misidentified as brain microvascular endothelium requires ETS factors to acquire vascular fate. Proc Natl Acad Sci $U$ S A 118. doi: http://dx.doi.org/10.1073/pnas.2016950118

Macosko EZ, Basu A, Satija R, Nemesh J, Shekhar K, Goldman M, Tirosh I, Bialas AR, Kamitaki N, Martersteck EM, et al. 2015. Highly Parallel Genome-wide Expression Profiling of Individual Cells Using Nanoliter Droplets. Cell 161: 1202-1214. doi: http://dx.doi.org/10.1016/j.cell.2015.05.002 Mansour AA, Goncalves JT, Bloyd CW, Li H, Fernandes S, Quang D, Johnston S, Parylak SL, Jin X, and Gage FH. 2018. An in vivo model of functional and vascularized human brain organoids. Nat Biotechnol 36: 432-441. doi: http://dx.doi.org/10.1038/nbt.4127

Mansour AA, Schafer ST, and Gage FH. 2021. Cellular complexity in brain organoids: Current progress and unsolved issues. Semin Cell Dev Biol 111: 32-39. doi: http://dx.doi.org/10.1016/j.semcdb.2020.05.013

Mariani J, Simonini MV, Palejev D, Tomasini L, Coppola G, Szekely AM, Horvath TL, and Vaccarino FM. 2012. Modeling human cortical development in vitro using induced pluripotent stem cells. Proc Natl Acad Sci U S A 109: 12770-12775. doi: http://dx.doi.org/10.1073/pnas.1202944109

Matsuoka RL, Rossi A, Stone OA, and Stainier DYR. 2017. CNS-resident progenitors direct the vascularization of neighboring tissues. Proc Natl Acad Sci USA 114: 10137-10142. doi: http://dx.doi.org/10.1073/pnas.1619300114

Mosser CA, Baptista S, Arnoux I, and Audinat E. 2017. Microglia in CNS development: Shaping the brain for the future. Prog Neurobiol 149-150: 1-20. doi: http://dx.doi.org/10.1016/j.pneurobio.2017.01.002

Muffat J, Li Y, Yuan B, Mitalipova M, Omer A, Corcoran S, Bakiasi G, Tsai LH, Aubourg P, Ransohoff RM, et al. 2016. Efficient derivation of microglia-like cells from human pluripotent stem cells. Nat Med 22: 1358-1367. doi: http://dx.doi.org/10.1038/nm.4189

Nostro MC, Cheng X, Keller GM, and Gadue P. 2008. Wnt, activin, and BMP signaling regulate distinct stages in the developmental pathway from embryonic stem cells to blood. Cell Stem Cell 2: 60-71. doi: http://dx.doi.org/10.1016/j.stem.2007.10.011

Ormel PR, Vieira de Sa R, van Bodegraven EJ, Karst H, Harschnitz O, Sneeboer MAM, Johansen LE, van Dijk RE, Scheefhals N, Berdenis van Berlekom A, et al. 2018. Microglia innately develop within cerebral organoids. Nat Commun 9: 4167. doi: http://dx.doi.org/10.1038/s41467-018-06684-2

Ottone C, Krusche B, Whitby A, Clements M, Quadrato G, Pitulescu ME, Adams RH, and Parrinello S. 2014. Direct cell-cell contact with the vascular niche maintains quiescent neural stem cells. Nat Cell Biol 16: 10451056. doi: http://dx.doi.org/10.1038/ncb3045

Ou MY, Ju XC, Cai YJ, Sun XY, Wang JF, Fu XQ, Sun Q, and Luo ZG. 2020. Heterogeneous nuclear ribonucleoprotein A3 controls mitotic progression of neural progenitors via interaction with cohesin. Development 147. doi: http://dx.doi.org/10.1242/dev.185132

Polioudakis D, de la Torre-Ubieta L, Langerman J, Elkins AG, Shi X, Stein JL, Vuong CK, Nichterwitz S, Gevorgian M, Opland CK, et al. 2019. A Single-Cell Transcriptomic Atlas of Human Neocortical Development during Mid-gestation. Neuron 103: 785-801 e788. doi: http://dx.doi.org/10.1016/j.neuron.2019.06.011

Qian T, Maguire SE, Canfield SG, Bao X, Olson WR, Shusta EV, and Palecek SP. 2017. Directed differentiation of human pluripotent stem cells to blood-brain barrier endothelial cells. Sci Adv 3: e1701679. doi: http://dx.doi.org/10.1126/sciadv.1701679 

165: 1238-1254. doi: http://dx.doi.org/10.1016/j.cell.2016.04.032

748 Quadrato G, Nguyen T, Macosko EZ, Sherwood JL, Min Yang S, Berger DR, Maria N, Scholvin J, Goldman M, Kinney JP, et al. 2017. Cell diversity and network dynamics in photosensitive human brain organoids. Nature 545: 48-53. doi: http://dx.doi.org/10.1038/nature22047 Salter MW, and Stevens B. 2017. Microglia emerge as central players in brain disease. Nat Med 23: 10181027. doi: http://dx.doi.org/10.1038/nm.4397

753 Shen Q, Goderie SK, Jin L, Karanth N, Sun Y, Abramova N, Vincent P, Pumiglia K, and Temple S. 2004.

754 Endothelial cells stimulate self-renewal and expand neurogenesis of neural stem cells. Science 304: 1338-

755 1340. doi: http://dx.doi.org/10.1126/science.1095505

756 Shi Y, Sun L, Wang M, Liu J, Zhong S, Li R, Li P, Guo L, Fang A, Chen R, et al. 2020. Vascularized human cortical organoids (vOrganoids) model cortical development in vivo. PLoS Biol 18: e3000705. doi: http://dx.doi.org/10.1371/journal.pbio.3000705

759 Smyth LCD, Rustenhoven J, Scotter EL, Schweder P, Faull RLM, Park TIH, and Dragunow M. 2018.

760 Markers for human brain pericytes and smooth muscle cells. J Chem Neuroanat 92: 48-60. doi: 761 http://dx.doi.org/10.1016/j.jchemneu.2018.06.001

762 Stern CD. 2005. Neural induction: old problem, new findings, yet more questions. Development 132: $2007-$ 763 2021. doi: http://dx.doi.org/10.1242/dev.01794

764 Sweeney MD, Zhao Z, Montagne A, Nelson AR, and Zlokovic BV. 2019. Blood-Brain Barrier: From 765 Physiology to Disease and Back. Physiol Rev 99: 21-78. doi: http://dx.doi.org/10.1152/physrev.00050.2017 766 Takebe T, Enomura M, Yoshizawa E, Kimura M, Koike H, Ueno Y, Matsuzaki T, Yamazaki T, Toyohara T, 767 Osafune K, et al. 2015. Vascularized and Complex Organ Buds from Diverse Tissues via Mesenchymal Cell768 Driven Condensation. Cell Stem Cell 16: 556-565. doi: http://dx.doi.org/10.1016/j.stem.2015.03.004

769 Takebe T, Sekine K, Enomura M, Koike H, Kimura M, Ogaeri T, Zhang RR, Ueno Y, Zheng YW, Koike N, 770 et al. 2013. Vascularized and functional human liver from an iPSC-derived organ bud transplant. Nature 499: 771 481-484. doi: http://dx.doi.org/10.1038/nature12271

772 Tata M, Wall I, Joyce A, Vieira JM, Kessaris N, and Ruhrberg C. 2016. Regulation of embryonic 773 neurogenesis by germinal zone vasculature. Proc Natl Acad Sci U S A 113: 13414-13419. doi: 774 http://dx.doi.org/10.1073/pnas.1613113113

775 Tata M, Wall I, Joyce A, Vieira JM, Kessaris N, and Ruhrberg C. 2016. Regulation of embryonic 776 neurogenesis by germinal zone vasculature. Proc Natl Acad Sci USA 113: 13414-13419. doi: 777 http://dx.doi.org/10.1073/pnas.1613113113

778 Thomsen ER, Mich JK, Yao Z, Hodge RD, Doyle AM, Jang S, Shehata SI, Nelson AM, Shapovalova NV, 779 Levi BP, et al. 2016. Fixed single-cell transcriptomic characterization of human radial glial diversity. Nat 780 Methods 13: 87-93. doi: http://dx.doi.org/10.1038/nmeth.3629

781 Tsai HH, Niu J, Munji R, Davalos D, Chang J, Zhang H, Tien AC, Kuo CJ, Chan JR, Daneman R, et al. 782 2016. Oligodendrocyte precursors migrate along vasculature in the developing nervous system. Science 351: 783 379-384. doi: http://dx.doi.org/10.1126/science.aad3839

784 Vanlandewijck M, He L, Mae MA, Andrae J, Ando K, Del Gaudio F, Nahar K, Lebouvier T, Lavina B, 785 Gouveia L, et al. 2018. A molecular atlas of cell types and zonation in the brain vasculature. Nature $\mathbf{5 5 4}$ 786 475-480. doi: http://dx.doi.org/10.1038/nature25739 
787 Wimmer RA, Leopoldi A, Aichinger M, Wick N, Hantusch B, Novatchkova M, Taubenschmid J, Hammerle

788 M, Esk C, Bagley JA, et al. 2019. Human blood vessel organoids as a model of diabetic vasculopathy. Nature

789 565: 505-510. doi: http://dx.doi.org/10.1038/s41586-018-0858-8

790 Xu Y, He Q, Wang M, Wang X, Gong F, Bai L, Zhang J, and Wang W. 2019. Quantifying blood-brain-

791 barrier leakage using a combination of evans blue and high molecular weight FITC-Dextran. $J$ Neurosci

792 Methods 325: 108349. doi: http://dx.doi.org/10.1016/j.jneumeth.2019.108349

793 Zadeh G, and Guha A. 2003. Angiogenesis in nervous system disorders. Neurosurgery 53: 1362-1374;

794 discussion 1374-1366. doi: http://dx.doi.org/10.1227/01.neu.0000093425.98136.31

795 Zhao Q, Eichten A, Parveen A, Adler C, Huang Y, Wang W, Ding Y, Adler A, Nevins T, Ni M, et al. 2018.

796 Single-Cell Transcriptome Analyses Reveal Endothelial Cell Heterogeneity in Tumors and Changes

797 following Antiangiogenic Treatment. Cancer Res 78: 2370-2382. doi: http://dx.doi.org/10.1158/0008-

798 5472.CAN-17-2728

799 Zhao Z, Nelson AR, Betsholtz C, and Zlokovic BV. 2015. Establishment and Dysfunction of the Blood-Brain

800 Barrier. Cell 163: 1064-1078. doi: http://dx.doi.org/10.1016/j.cell.2015.10.067

801 Zilionis R, Nainys J, Veres A, Savova V, Zemmour D, Klein AM, and Mazutis L. 2017. Single-cell barcoding

802 and sequencing using droplet microfluidics. Nat Protoc 12: 44-73. doi:

803 http://dx.doi.org/10.1038/nprot.2016.154

804 Zlokovic BV. 2011. Neurovascular pathways to neurodegeneration in Alzheimer's disease and other 805 disorders. Nat Rev Neurosci 12: 723-738. doi: http://dx.doi.org/10.1038/nrn3114

806 Zudaire E, Gambardella L, Kurcz C, and Vermeren S. 2011. A computational tool for quantitative analysis

807 of vascular networks. PLoS One 6: e27385. doi: http://dx.doi.org/10.1371/journal.pone.0027385 


\section{$809 \quad$ Figure Legends}

810 Figure 1. Generation of an in vitro model of vessel organoids. (A) Schematic view of 811 the methods for generating VOrs from GFP-hESC. EBs: embryonic bodies; VPs: vascular 812 progenitors; VOr: vessel organoid. (B) Different developmental stages of VOrs from D4 813 to D20. Top, right field; bottom, GFP. Scale bar, $200 \mu \mathrm{m}$. (C) qPCR analysis for expression 814 of stem markers (NANOG, OCT4) and vessel markers (PECAM1, VE-Cadherin, VWF, $815 V E G F R 1, V E G F R 2, P D G F R$ ) in developing VOrs, using GAPDH as internal control. Data 816 are presented as mean \pm SEM ( $\mathrm{n}=3$ independent experiments). (D) Immunostaining of 817 GFP and CD31 in D40 VOrs. Scale bar, $200 \mu \mathrm{m}$. Bottom: Imaris reconstruction of VOrs 818 showing integrated vasculature structures. (E) Immunostaining of GFP and CD31 for the 819 vascular structures in VOrs. Scale bar, $20 \mu \mathrm{m}$. Top right: Section view in VOr showing the 820 lumen structure. (F) Immunostaining of CD31 and PDGFR $\beta$ for endothelial cells and 821 pericytes, respectively. Scale bar, $50 \mu \mathrm{m}$.

823 Figure 1-figure supplement 1. VOrs recapitulate human vessel development. (A) 824 Morphological appearance of VOrs at D40. Scale bar, $500 \mu \mathrm{m}$. (B) Quantification of the 825 VOr diameter from D4 to D40. Data are mean \pm SEM of $10-21$ organoids in each time 826 points. (C) Flow cytometry plots of temporal development of $\mathrm{CD}^{2} 1^{+} \mathrm{GFP}^{+}$cells in 827 differentiating VOrs. (D) Immunostaining of GFP and CD31 for the vascular angiogenesis 828 structures. Scale bar, $20 \mu \mathrm{m}$. (E) Quantification of the average vessel length (E1), total 829 number of junctions (E2) and mean E lacunarity (E3) for D40 VOrs. (F) Up-take of 830 acetylated low-density lipoprotein (DiI-Ac-LDL) of VOrs at indicated time points. Scale 831 bar, $20 \mu \mathrm{m}$. 
833 Figure 2. Single-cell transcriptomic analysis of vessel organoids. (A) UMAP plot

834 showing the nine major cell types isolated from D40 VOrs. (B) Violin plots showing the

835 expression value of the typical markers in each cluster. (C) Expression pattern of cell-type

836 specific markers in VOrs. Relative expression level is plotted from gray (low) to blue (high)

837 colors. (D) Immunostaining of $\alpha$ SMA for representing the smooth muscle cells in VOrs.

838 Scale bar, $10 \mu \mathrm{m}$. (E) Immunostaining of PDGFR $\beta$ for representing the pericytes in VOrs.

839 Scale bar, $10 \mu \mathrm{m}$. (F) Immunostaining of microglia markers TREM2 and TMEM119 in

840 VOrs at D40. Scale bar, $50 \mu \mathrm{m}$. (G) Violin plots showing the expression value of the venous

841 marker EPHB4 and arterial marker DLL4 in EC clusters. (H) Expression pattern of arterial

842 and venous markers in EC clusters. Relative expression level is plotted from gray to green

843 (EPHB4) or red (DLL4) colors.

Figure 2-figure supplement 1. Cell type analysis for VOrs by scRNA-seq and immunostaining. (A) Correlation analysis of scRNA-seq data from two batches of VOr samples. (B) Proportions of cell types among all the cells from VOrs. (C) Heatmap showing the top five most enriched genes for each cell type. (D) Immunostaining of DLL4 for labeling the arterial endothelial cells in VOrs. Scale bar, $10 \mu \mathrm{m}$. (E) Immunostaining of EPHB4 for labeling the venous endothelial cells in VOrs. Scale bar, $10 \mu \mathrm{m}$.

Figure 3. Cell fate trajectory analysis in VOrs and the comparison with cell types in

853 vivo. (A) Single-cell trajectories by monocle analysis showing developmental stage of the

854 VOrs. (B) Clusters in UMAP showing trajectory track. (C) Developmental trajectory of 855 indicated cell clusters in VOrs. (D) Heatmap showing the expression level of the main cell

856 type-specific markers with pseudo-time. (E) Expression of markers in SMC and PC with 857 pseudo-time. (F) Correlation analysis of cell clusters (EC, MG, PC, SMC, FB) between 858 VOrs and mouse brain. V, data from VOrs; M, data from mouse. (G) Correlation analysis 859 of cell clusters (EC, MG, PC) among VOrs, mouse and human brain single-cell data. V, 860 data from VOrs; $M$, data from mouse; H, data from human. (H) Venn diagram showing the 861 DEGs in EC clusters for VOr and human samples compared to mouse samples. Red for up- 
862 regulated genes, black for down-regulated genes. (I) Heat-map showing the top enriched

863 DEGs in the EC cluster for VOrs samples compared to mouse sample (fold change > 1.25

864 and $\mathrm{p}<0.05)$. (J) GO analysis of the 149 up-regulated DEGs in (h) (p value < 0.1 and FDR

$865<0.05$ ). (K) Top 20 marker genes for VOrs in the main clusters (EC, PC, MG) (fold

866 change $>1.25$ and $\mathrm{p}<0.05)$ compared to human sample, with significantly pathways by

867 GO analysis ( $\mathrm{p}$ value $<0.1$ and FDR < 0.05 ). V, data from VOrs; H, data from human.

868

869

Figure 3-figure supplement 1. Cell types in VOrs are similar to that of human

870 samples in vivo. (A) Trajectory analysis showing five main developmental stages. (B)

871 UMAP plot showing five developmental stages of VOrs. (C) Expression of markers in four

872 main clusters with pseudo-time. (D) Venn diagram showing the DEGs in PC clusters for

873 VOr and human samples compared with mice samples. Red for up-regulated genes, black

874 for down-regulated genes. (E) Heat-map showing the top enriched common DEGs in the

875 PC cluster for VOrs samples compared to mouse sample (fold change $>1.25$ and $\mathrm{p}<0.05$ ).

876 (F) Principal component analysis for relative relationship of 29 distinct cell samples from

877 published datasets, including primary ECs, iECs (induced ECs), and Epi-iBMECs

878 (neuroectodermal epithelial lineage-induced brain microvascular endothelial cells) at

879 various days of differentiation and hPSCs (human pluripotent stem cells). ECs in VOrs are

880 highlighted to emphases the similarity to ECs rather than iBMECs. (G) Heat-map

881 illustrating differences in expression levels of both endothelial- and epithelial-specific

882 genes in all cell samples from $(\mathbf{F})$. The VOR sample (circled) shows more EC-specific gene

883 expression rather than epithelial-specific expression. 
Figure 4. Generation of fVBOrs with BBB structure. (A) Schematic view of the method

886 for generating fVBOrs. EBs, embryonic bodies; NE, neuroepithelium; VP, vascular

887 progenitor; VO, vessel organoid; BOr, brain organoid; fVBOr, fusion vascularized brain

888 organoid. (B) fVBOrs at different developmental stages. Scale bar, $500 \mu \mathrm{m}$. V, VOr; B,

889 BOr. (C) Immunostaining of CD31 and DCX for labeling vessels and neurons,

890 respectively, in D40 fVBOrs. Scale bar, $200 \mu \mathrm{m}$. (D) Immunostaining of CLDN5 for

891 labeling tight junctions in fVBOrs. Scale bar, $200 \mu \mathrm{m}$. D1, enlarged area. (E)

892 Immunostaining for markers of astrocytes (S100/GFAP), neurons (MAP2), endothelial

893 cells (CD31) and vessel structures (GFP) in fVBOrs. Orange arrows indicate astrocytes

894 end feet. Scale bar, $20 \mu \mathrm{m}$. (F, G) Confocal fluorescence images showing the transport of

895 rhodamine-labeled angiopep-2 (Angiopep-2-Rhod), rhodamine-scramble peptide

896 (Scramble-Rhod) in fVBOrs. Scale bar, $200 \mu \mathrm{m}$. Bottom, z-stack images of rhodamine

897 signals.

Figure 4-figure supplement 1. Generation of human brain organoids. (A) Schematic workflow for generating BOrs from hESC. EBs, embryonic bodies; NE, neuroepithelium;

901 BOr, brain organoid. (B) Immunostaining for cortical progenitor markers PAX6 and p-

902 VIM in BOrs at D30. Scale bar, $50 \mu \mathrm{m}$. (C) Immunostaining for young neuron marker 903 DCX, proliferation marker KI67, intermediate progenitor marker TBR2 and neuron marker 904 TUJ1 in D50 BOrs. Scale bar, $50 \mu \mathrm{m}$. (D, E) Immunostaining for cortical layer markers 905 TBR1, CTIP2, SATB2 and REELIN in D60 BOrs. Scale bar, $50 \mu \mathrm{m}$. (F) Immunostaining 906 for EC marker CD31 in BOrs at D25 and D45. Scale bar, $200 \mu \mathrm{m}$.

908 Figure 4-figure supplement 2. BBB-like structures in fVBOrs. (A) Fluorescence 909 image showing the expression of CLDN5 in fVBOrs and BOrs respectively. Scale bar, 50 $910 \mu \mathrm{m}$. (B) Fluorescence image showing the expression of tight junctions marker ZO-1 in 911 fVBOrs at D40. Scale bar, $50 \mu \mathrm{m}$. (C) Fluorescence image showing the expression of the 912 efflux transporter p-Glycoprotein in fVBOrs at D40. Scale bar, $50 \mu \mathrm{m}$. (D) TEM of vascular 913 and BBB structure in D80-fVBOr. Arrows: basement membrane; p: pericyte; a: end-foot 
914 of astrocyte. Scale bar, $1 \mu \mathrm{m}$. (E) Confocal z-stack images of rhodamine-labeled angiopep-

9152 (Angiopep-2-Rhod) in D40 BOrs. (F) Quantification for the intensity of Angiopep-2-

916 Rhod across different z-stack images of fVBOrs compared with BOrs. Data are presented

917 as mean \pm SEM ( $=3$ organoids in each sample). ${ }^{*} \mathrm{p}<0.05$, paired t-test.

919 Figure 5. Microglial cells in fVBOrs. (A) UMAP plot showing single-cell expression 920 pattern of microglial specific markers in VOr. Relative expression level is plotted from 921 gray to blue colors. (B) GO analysis of microglial cell marker genes (p value $<0.1$ and 922 FDR < 0.05). (C) qPCR analysis for expression of microglial markers AIF1 and TMEM119 923 in developing VOrs. Data are presented as mean \pm SEM $(n=3$ independent experiments 924 with $6-7$ organoids in each group at indicated time point). ${ }^{*} * \mathrm{p}<0.01, * * * \mathrm{p}<0.001$. (D)

925 Immunostaining of IBA1 for labeling microglial cells in D25 and D40 VOrs. Scale bar, $926200 \mu \mathrm{m}$. (E) Quantification of the IBA1 ${ }^{+}$cell number in D25 and D40 VOrs. $\mathrm{n}=16$. 927 Students t-test, $* * * \mathrm{p}<0.001$. (F) Immunostaining of IBA1 for labeling microglial cells in 928 BOrs and fVBOrs, respectively. Scale bar, $200 \mu \mathrm{m}$. (G) qPCR analysis for the expression 929 of indicated genes in D40 fVBOrs treated with LPS (500 ng/ml, MCE, HY-D1056) without 930 or with PLX5622 $2 \mu \mathrm{M}$ (MCE, HY-11415) using DMSO as vehicle control. Relative 931 expression was normalized to GAPDH. $\mathrm{n}=3$ independent experiments with 8-10 organoids 932 in each group. One-way ANOVA, ${ }^{*} \mathrm{p}<0.05, * * \mathrm{p}<0.01$.

934 Figure 5-figure supplement 1. PLX5622 ablates MGs in VOrs. (A) IBA1 staining in

935 VOrs showing ablation of microglial cells after treatment with $2 \mu \mathrm{M}$ PLX5622 for 7 days, 936 and drug withdraw for 3 days. Scale bar, $200 \mu \mathrm{m}$. (B) Quantification of microglial cell

937 numbers in VOrs with indicated treatments. Data are shown as mean $\pm \operatorname{SEM}(\mathrm{n}=3$

938 independent experiments with 8-10 organoids in each group) One-way ANOVA, ***p <

939 0.001, ns, no significant difference. (C) Ablation of microglia in fVBOrs treated with 2

$940 \mu \mathrm{M}$ PLX5622 for 7 days. Scale bar, $200 \mu \mathrm{m}$. 
942 Figure 6. Increased neurogenesis in fVBOrs. (A) Immunostaining for DAPI showing the 943 neuroepithelium rosettes of BOrs and fVBOrs at D25. Scale bar, $50 \mu \mathrm{m}$. (B) Quantification 944 of neuroepithelium thickness of BOrs and fVBOrs. Data are presented as mean \pm SEM 945 (BOrs: $\mathrm{n}=60$ rosettes from 7 organoids; fVBOrs: $\mathrm{n}=55$ rosettes from 6 organoids). Two946 tailed Student's $t$-test. ***p < 0.001. (C) Immunostaining for PAX6 and p-VIM in VZ-like 947 area of BOrs and fVBOrs at D25. Scale bar, $50 \mu \mathrm{m}$. Apical membrane was showed in 948 yellow circle. (D, E) Quantification of the density of $\mathrm{PAX6}^{+}(\mathbf{D})$ and the density of p-VIM ${ }^{+}$ 949 cells per $100 \mu \mathrm{M}$ apical membrane length (E) in BOrs and fVBOrs. Data are presented as 950 mean \pm SEM (PAX6: $\mathrm{n}=25$ rosettes from 4 organoids; $\mathrm{p}$-VIM: $\mathrm{n}=23$ rosettes from 4 951 organoids). Two-tailed Student's $t$-test. ***p $<0.001$. (F) Immunostaining for DCX in 952 BOrs and fVBOrs at D25. Scale bar, $50 \mu \mathrm{m}$. (G) Quantification of the intensity of DCX in 953 BOrs and fVBOrs. Data are presented as mean \pm SEM (BOrs: $n=24$ rosettes from 3 954 organoids, fVBOrs: $\mathrm{n}=15$ rosettes from 4 organoid $)$. $\mathrm{ns}$, no significant difference $(\mathrm{p}=$ 955 0.308, two-tailed Student's $t$-test).

957 Figure 6-figure supplement 1. Reduced apoptotic areas in fVBOrs. (A) 958 Immunostaining for early born deep-layer neuronal marker TBR1 in BOrs and fVBOrs at 959 D40. Scale bar, $50 \mu \mathrm{m}$. (B) Quantification of TBR1 ${ }^{+}$cells in BOrs and fVBOrs (BOr: $\mathrm{n}=$ 96016 rosettes from 6 organoids, fVBOr: $n=8$ rosettes from 3 organoids). Data are presented 961 as mean \pm SEM (two-tailed Student's $t$-test, $\mathrm{p}=0.8340)$. $(\mathbf{C}, \mathbf{D})$ Staining of cleaved962 caspase3 (c-CASP3) in BOrs or fVBOrs at D40 (C) and D70 (D). Scale bar, $200 \mu \mathrm{m}$. (E) 963 Quantification of the cleaved-caspase3 intensity in BOrs and fVBOrs at D40 and D70, 964 respectively. Data are presented as mean \pm SEM of chosen fields of 5 organoids in each 965 sample. ${ }^{*} \mathrm{p}<0.05$ ( $\mathrm{p}=0.0303$ for $\mathrm{D} 40, \mathrm{p}=0.0286$ for D70, two-tailed Student's $t$-test). 
bioRxiv preprint doi: https://doi.org/10.1101/2022.01.04.474960; this version posted January 4, 2022. The copyright holder for this preprint (which was not certified by peer review) is the author/funder, who has granted bioRxiv a license to display the preprint in perpetuity. It is made available under aCC-BY 4.0 International license.

\section{Movie Supplement 1}

968 PBS fluid was microinjected into the vessel-like lumen in D40 VOr with continuous

969 pressure, showing liquid flow and vessel wall expansion without leakage. Electrode inner

970 diameter: $500 \mathrm{~nm}$.

971

972 Supplementary File 1. Key resources table 


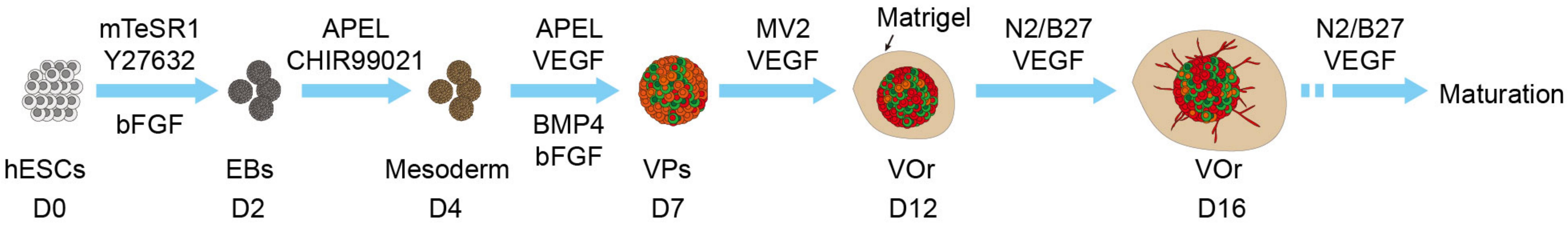

B

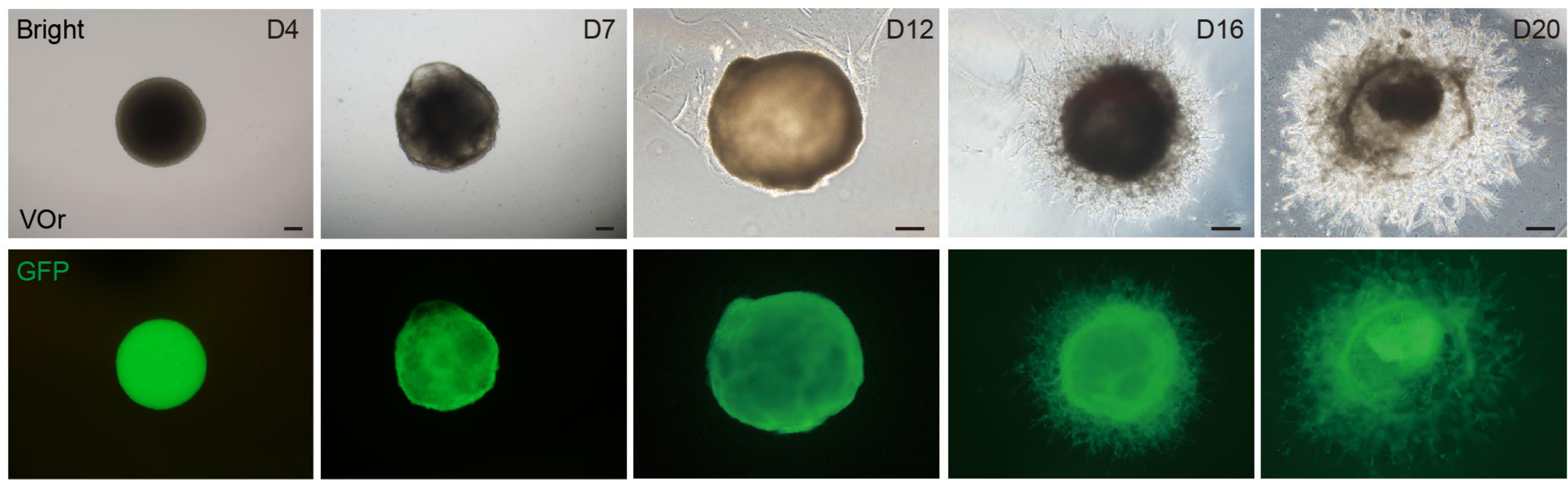

C

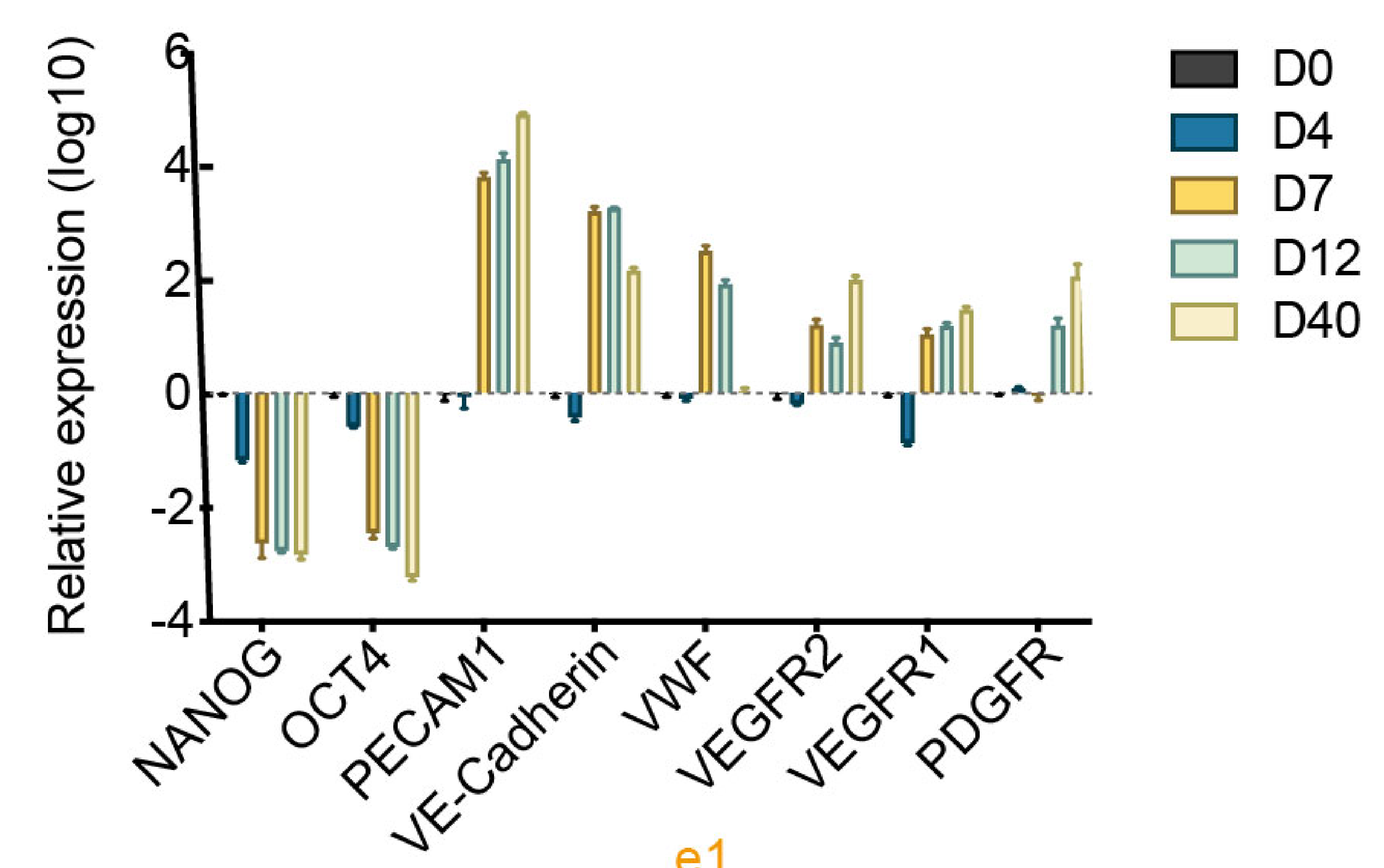

E

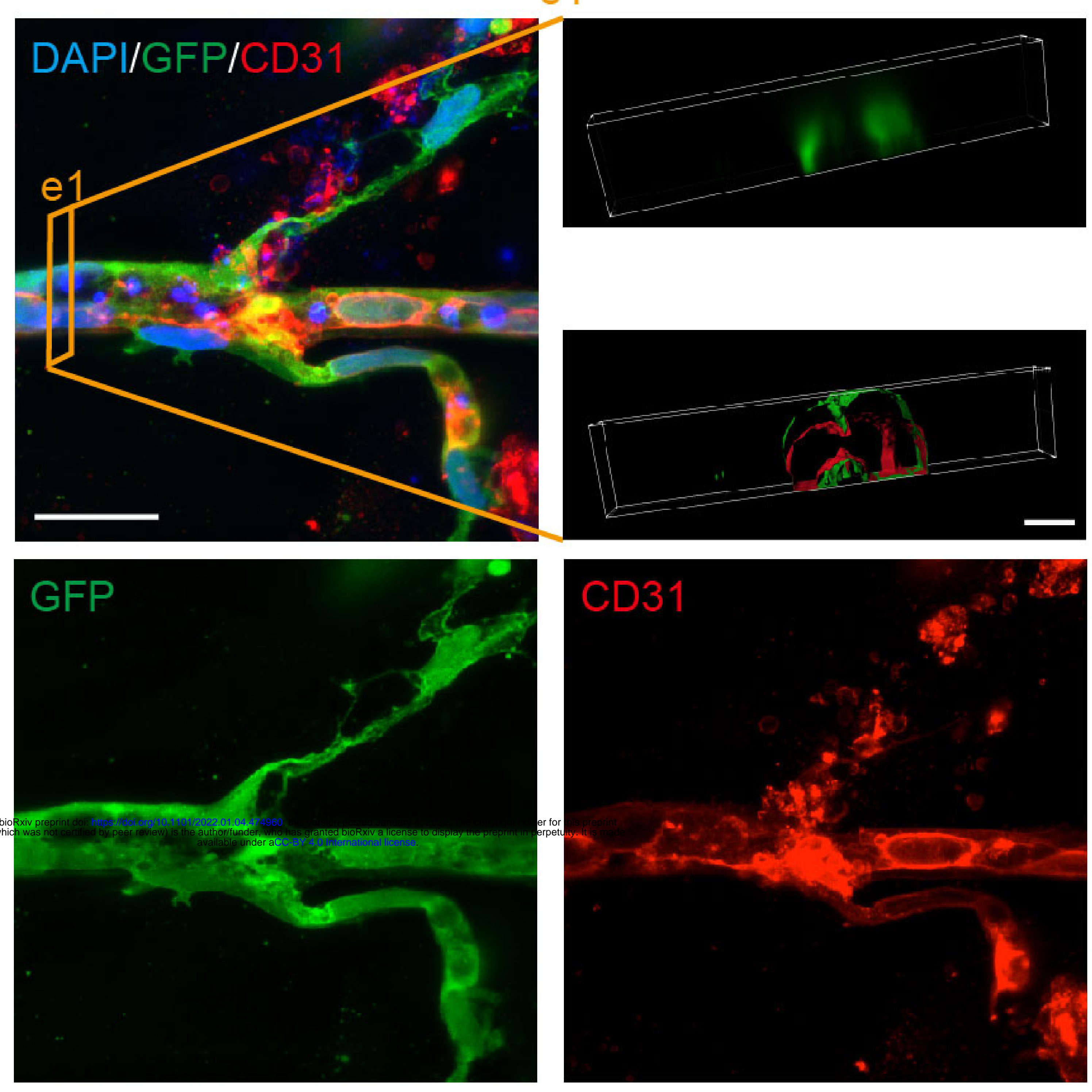

norge

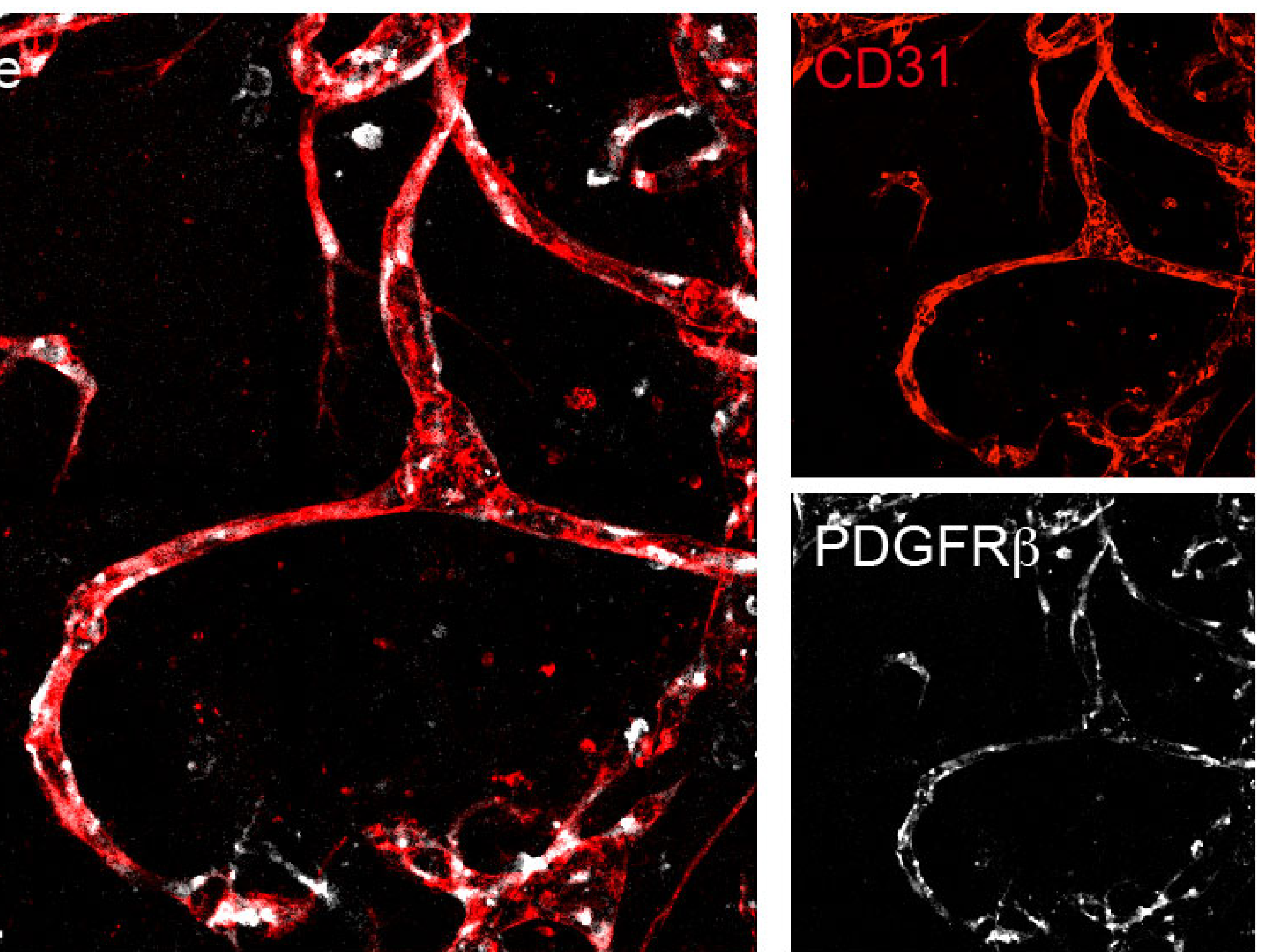

D
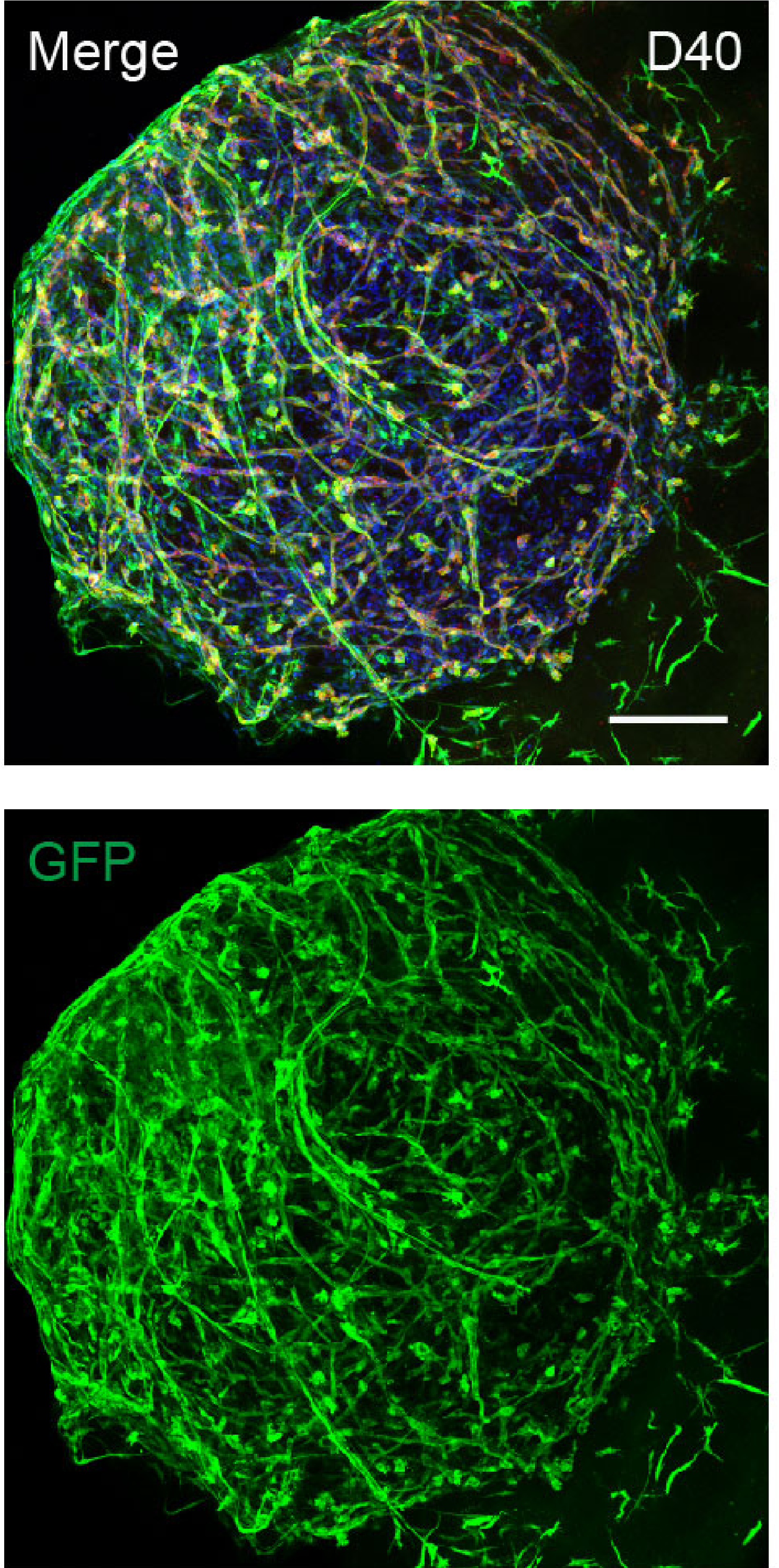

DAPI

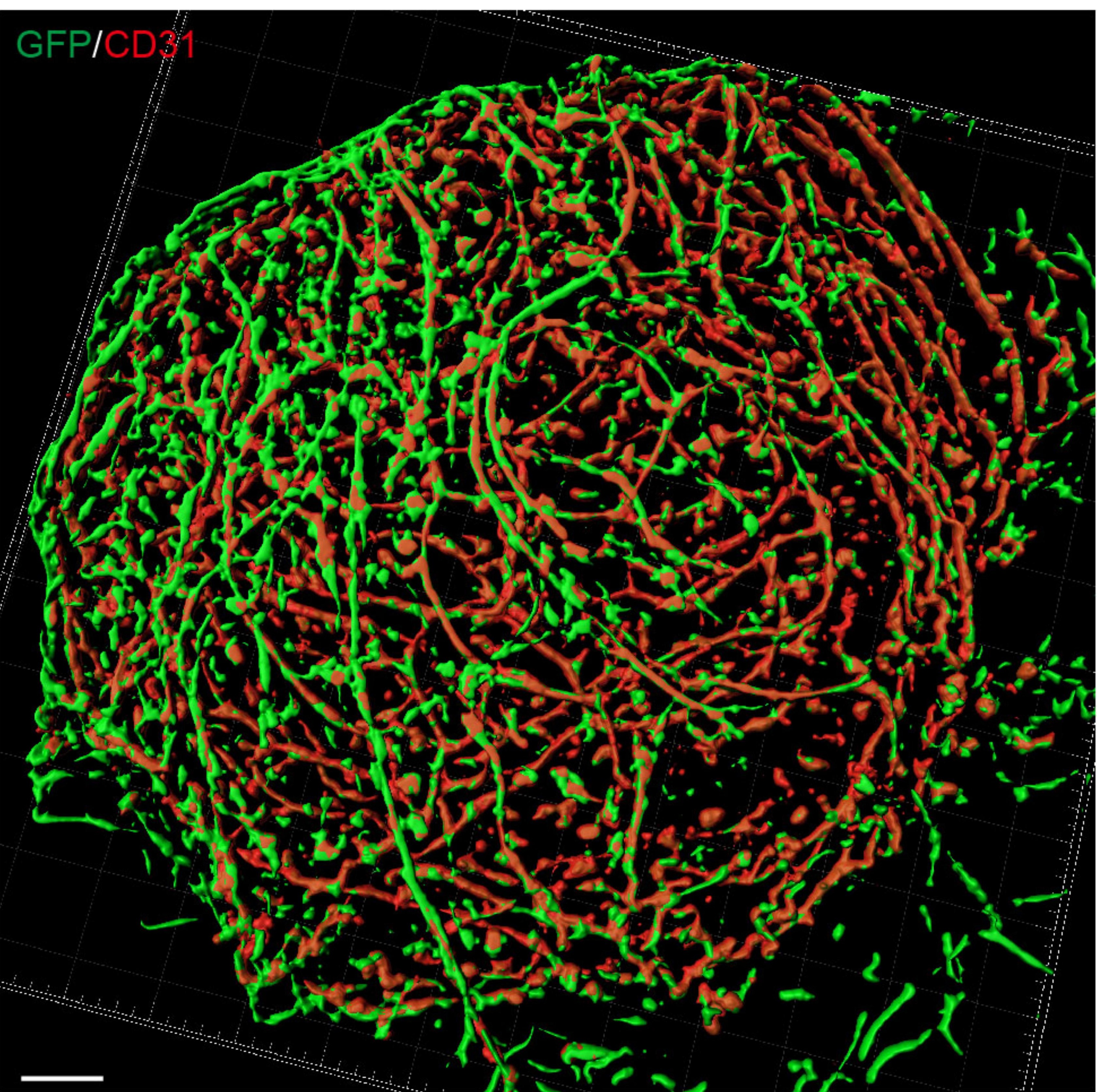


A $\quad$ B

B
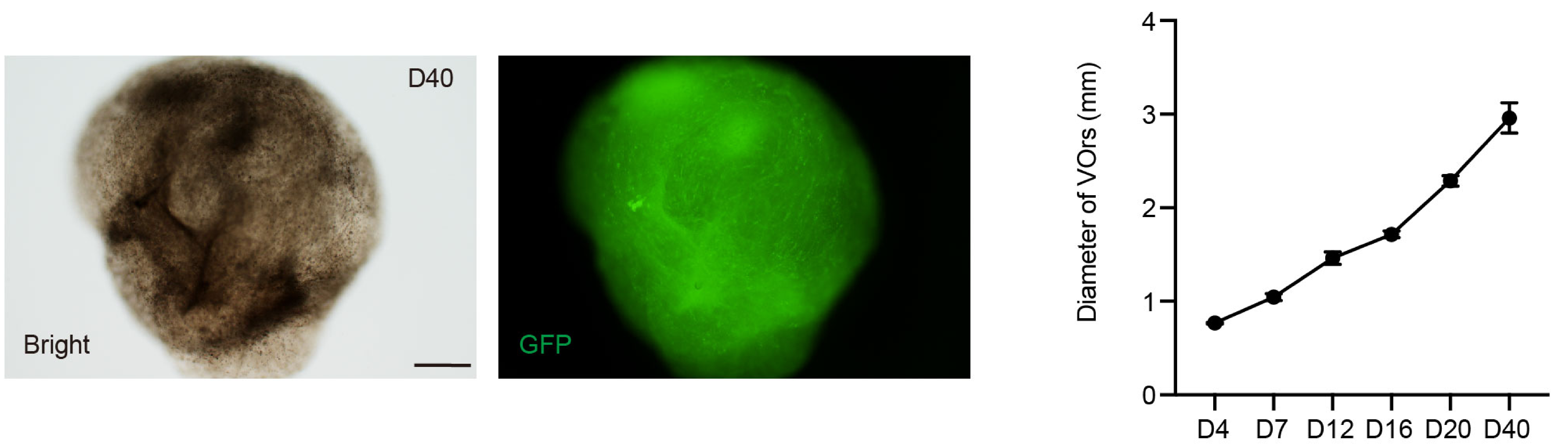

C

DO (hES)

D4-Vor

D7-VOr

D12-VOr

D20-VOr

D40-VOr
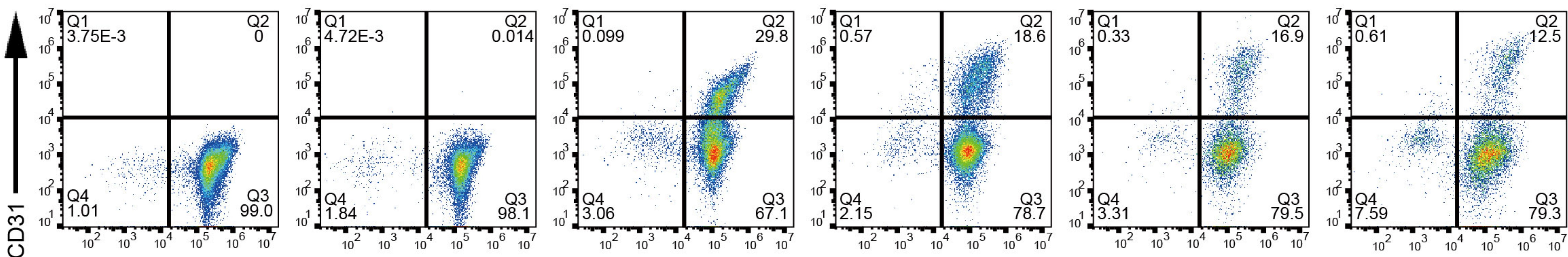
GFP
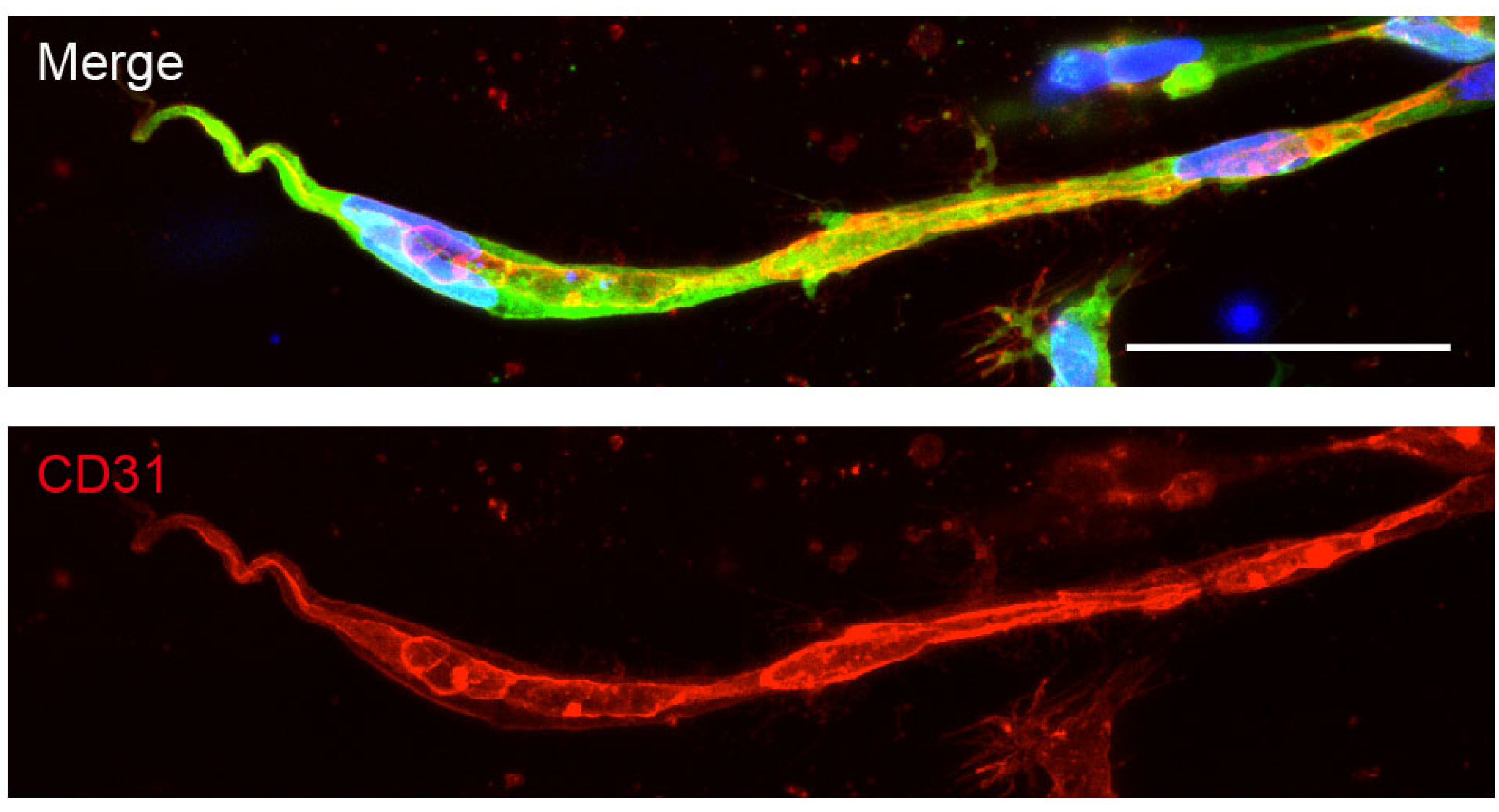

E

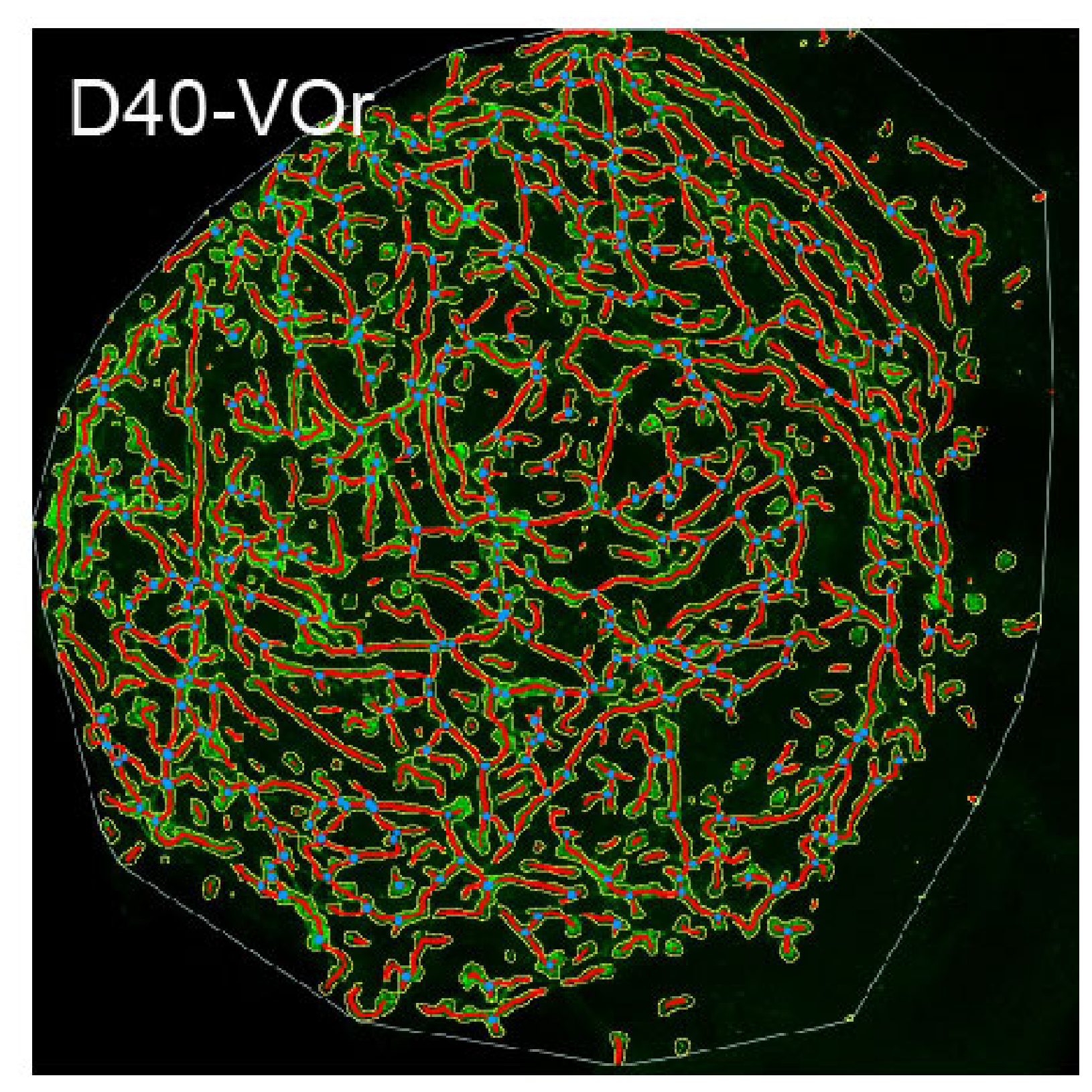

E1
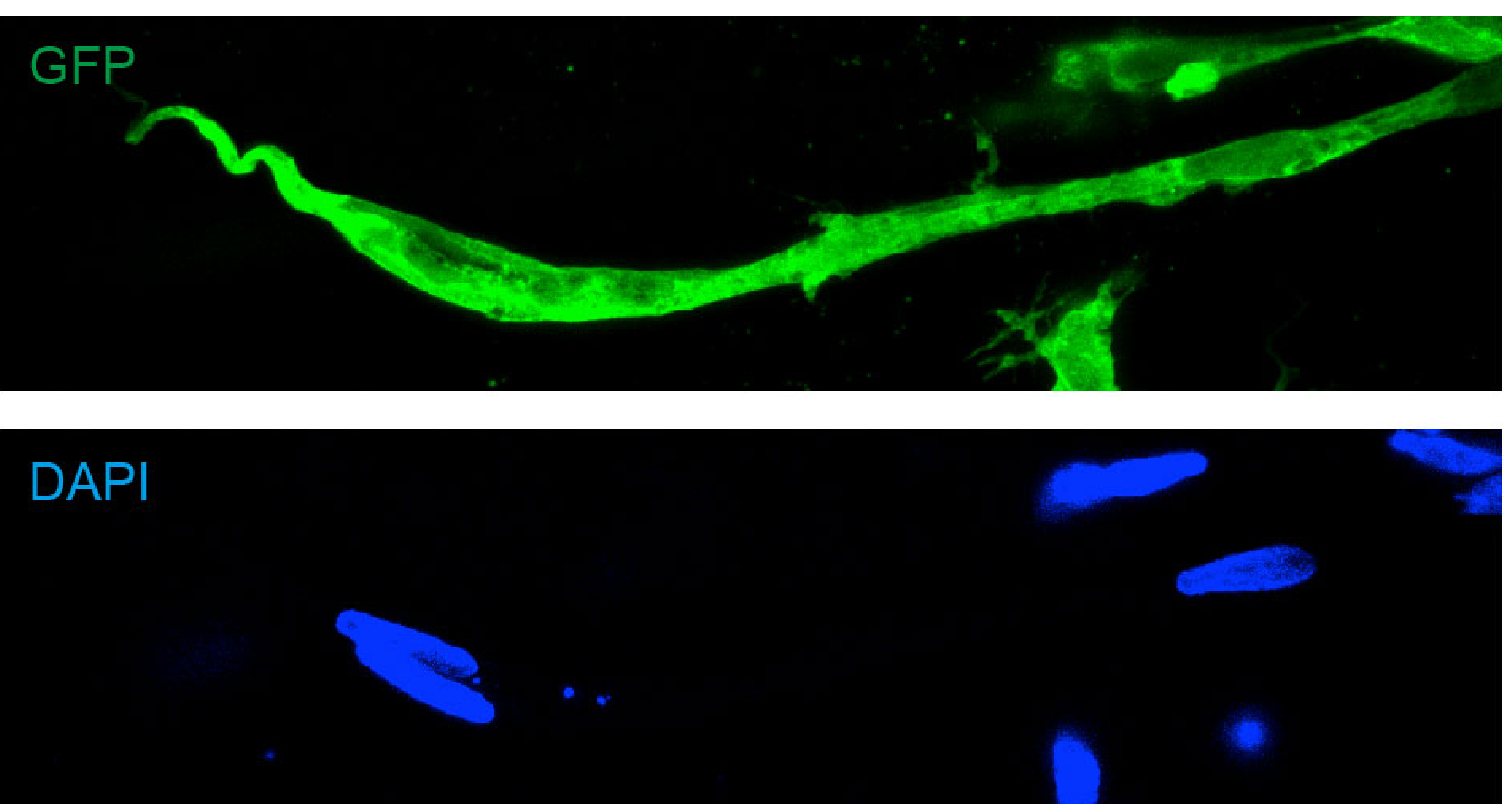

E2
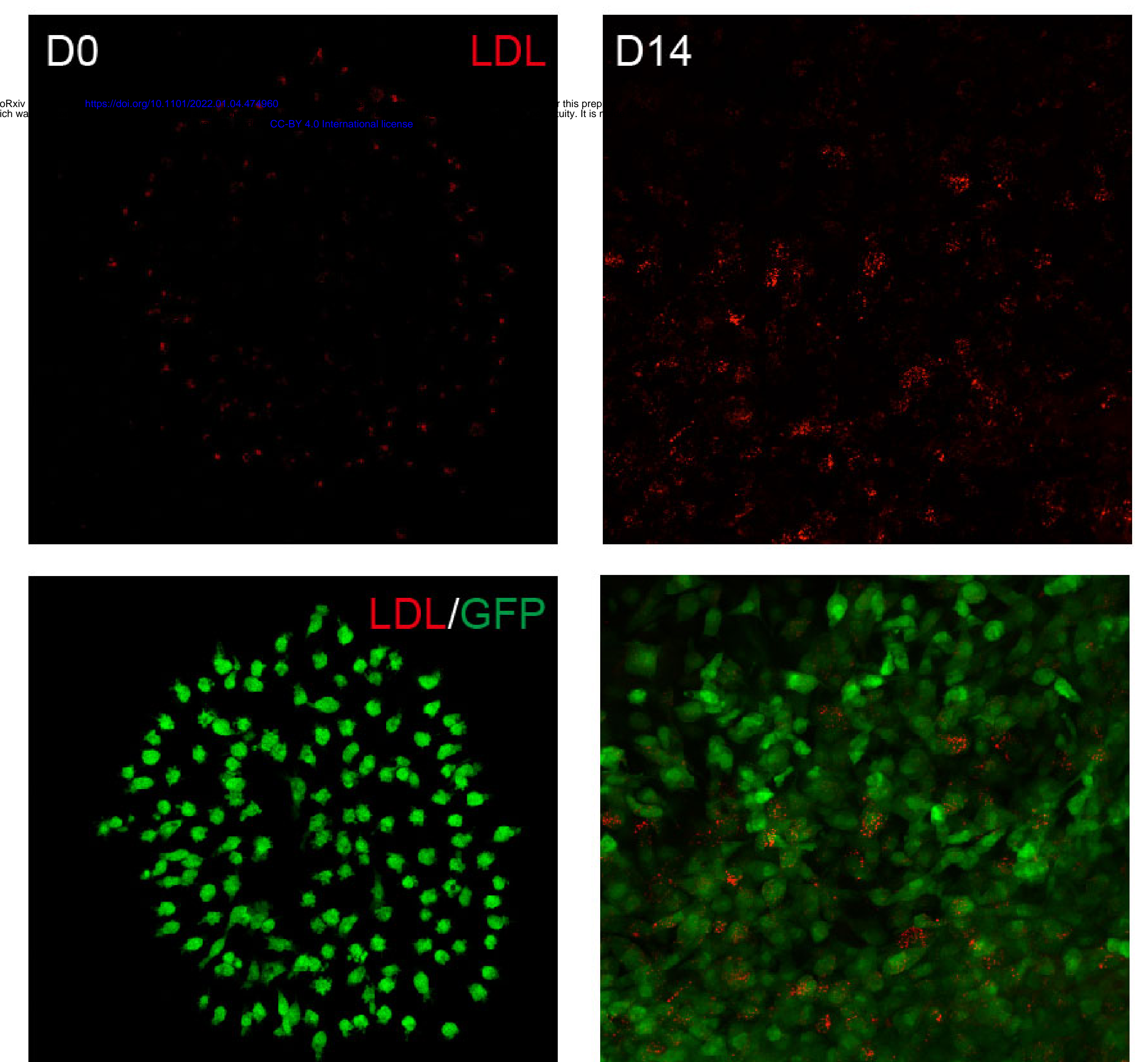

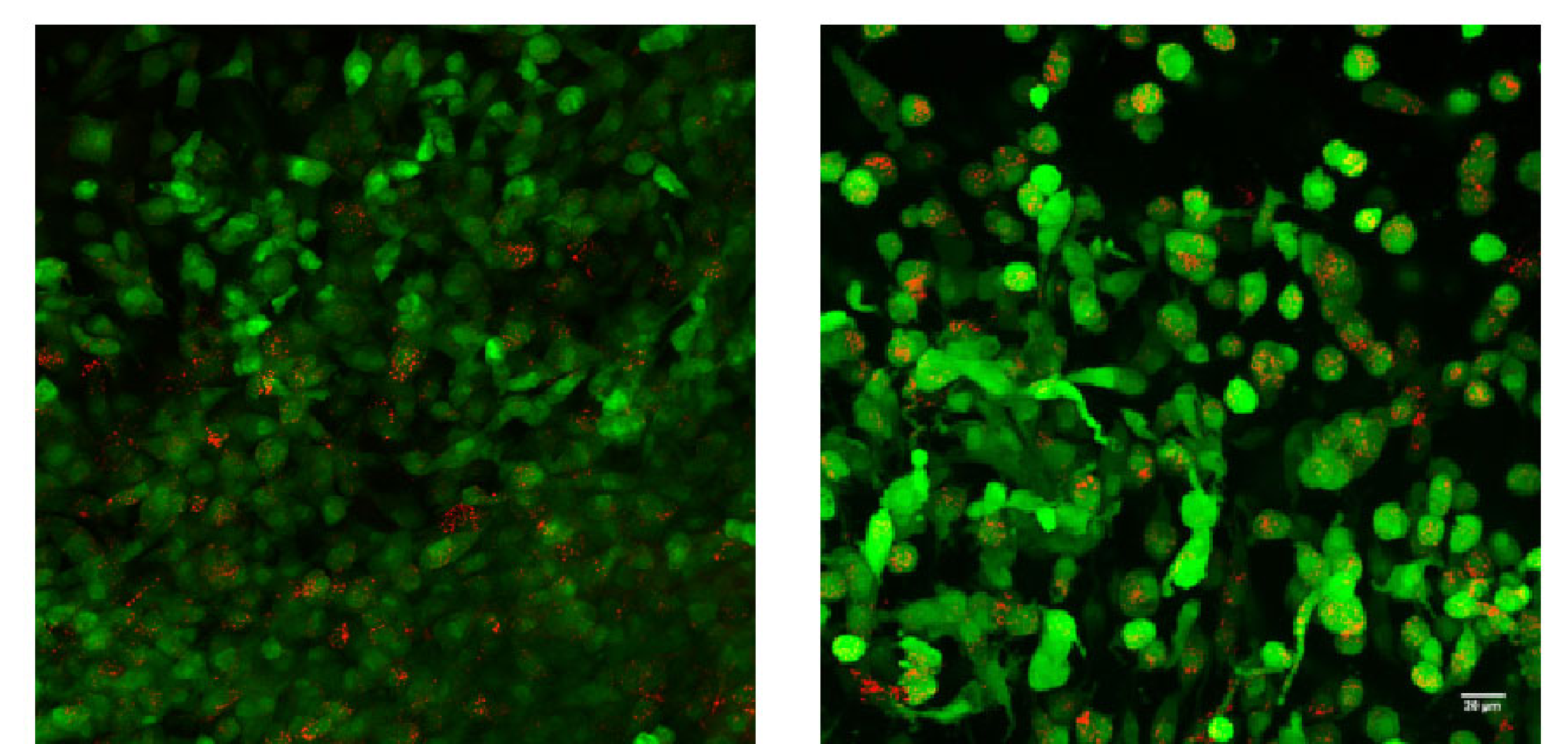

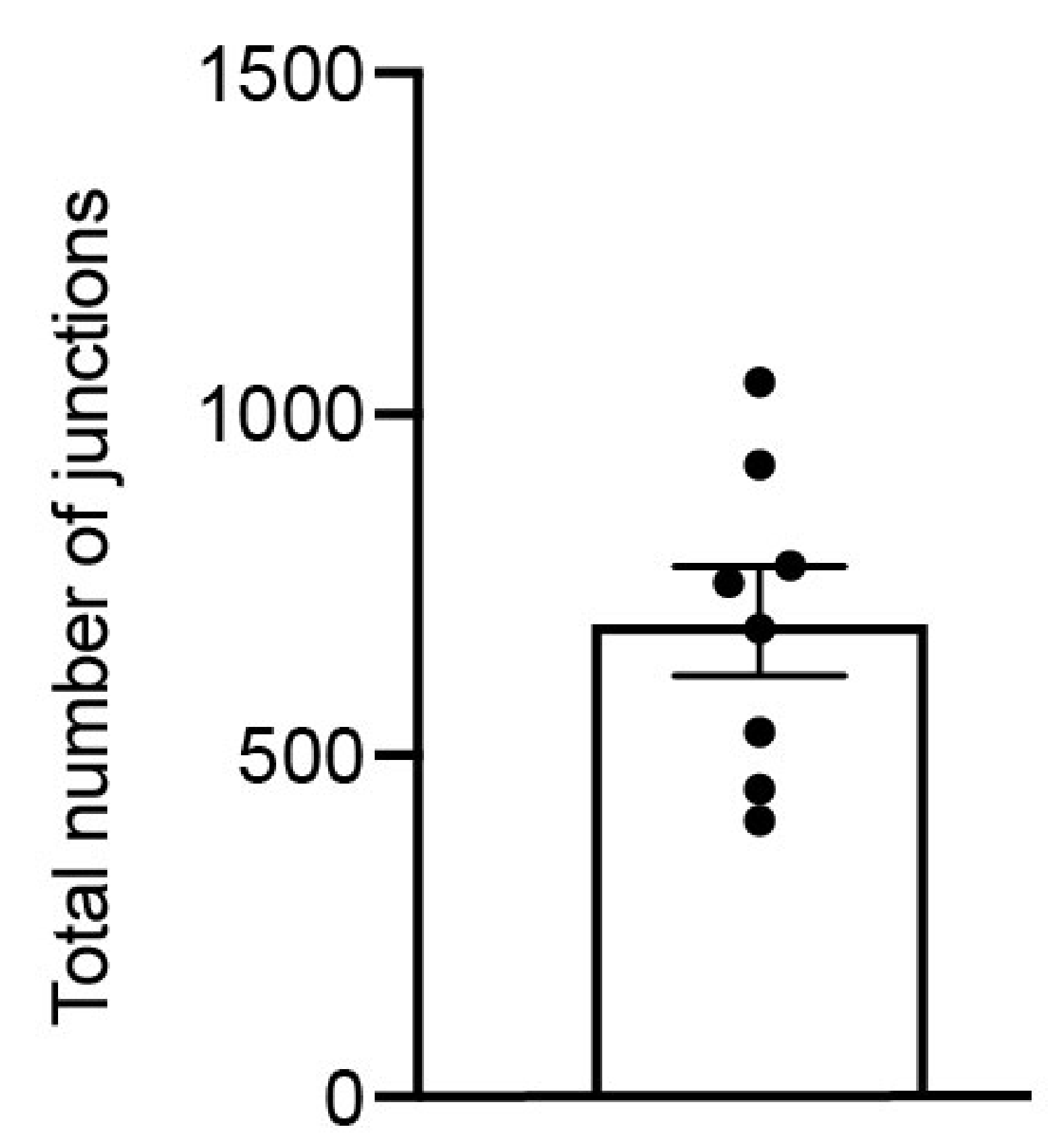

D18
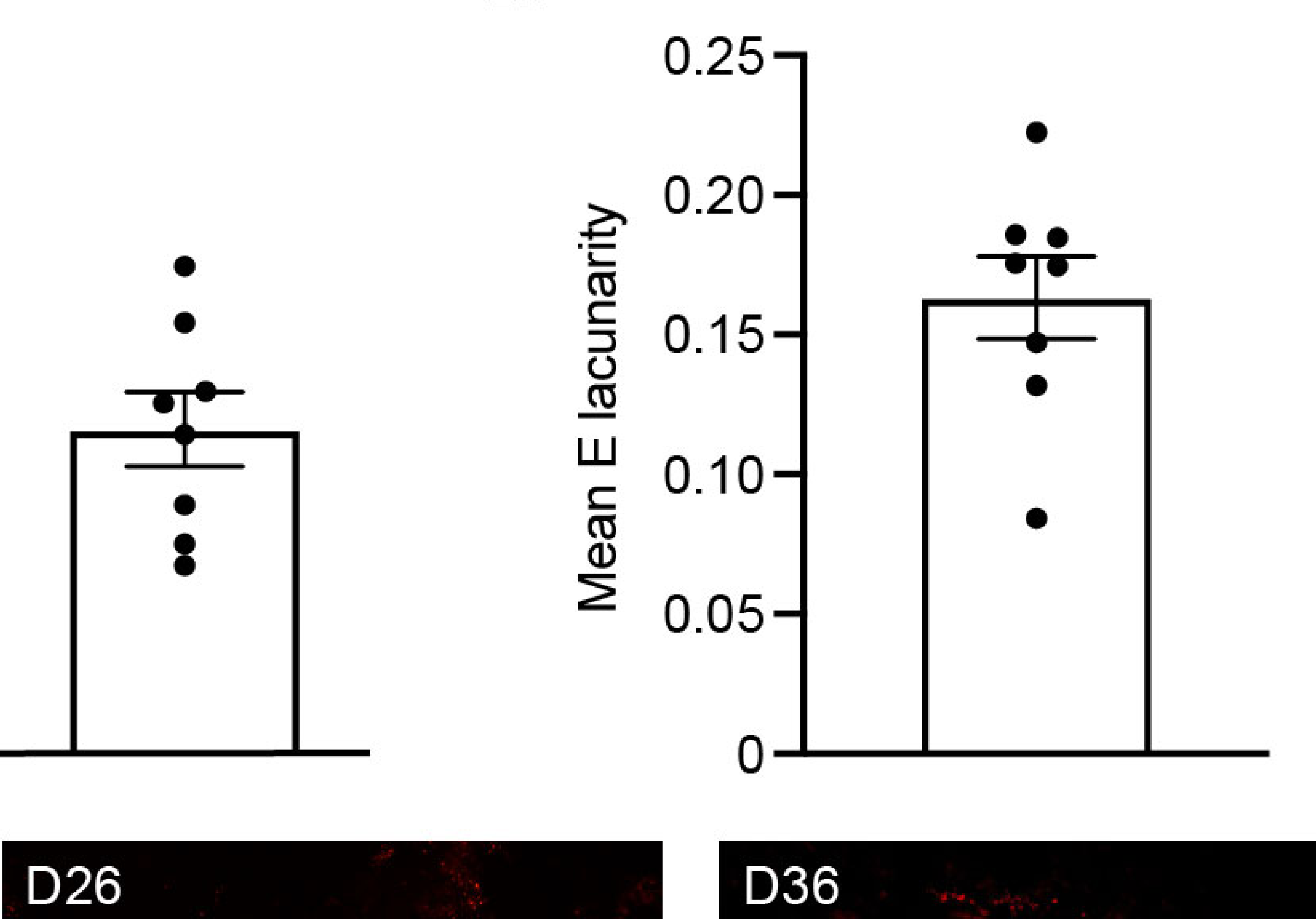

D36

26

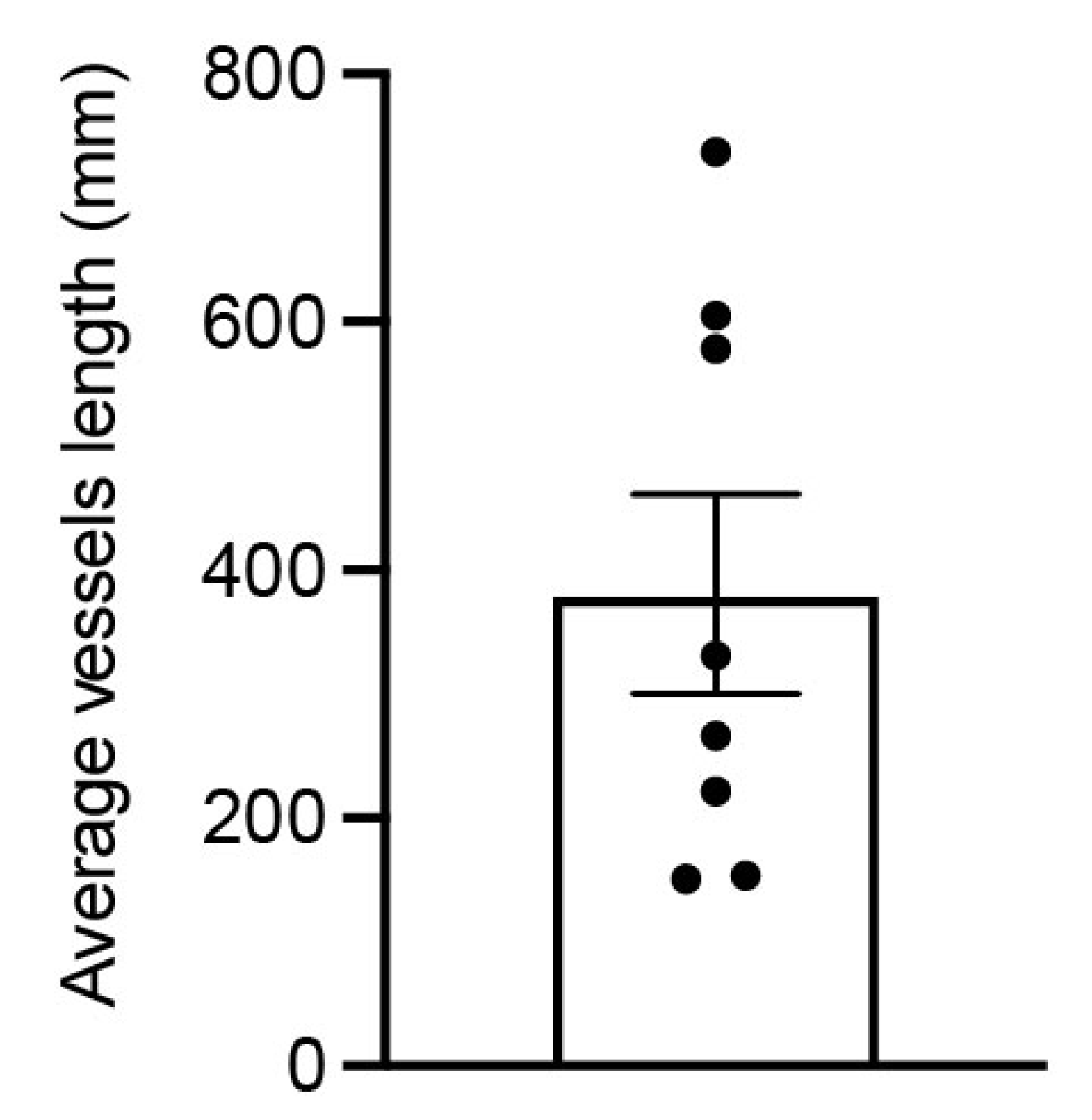

E3
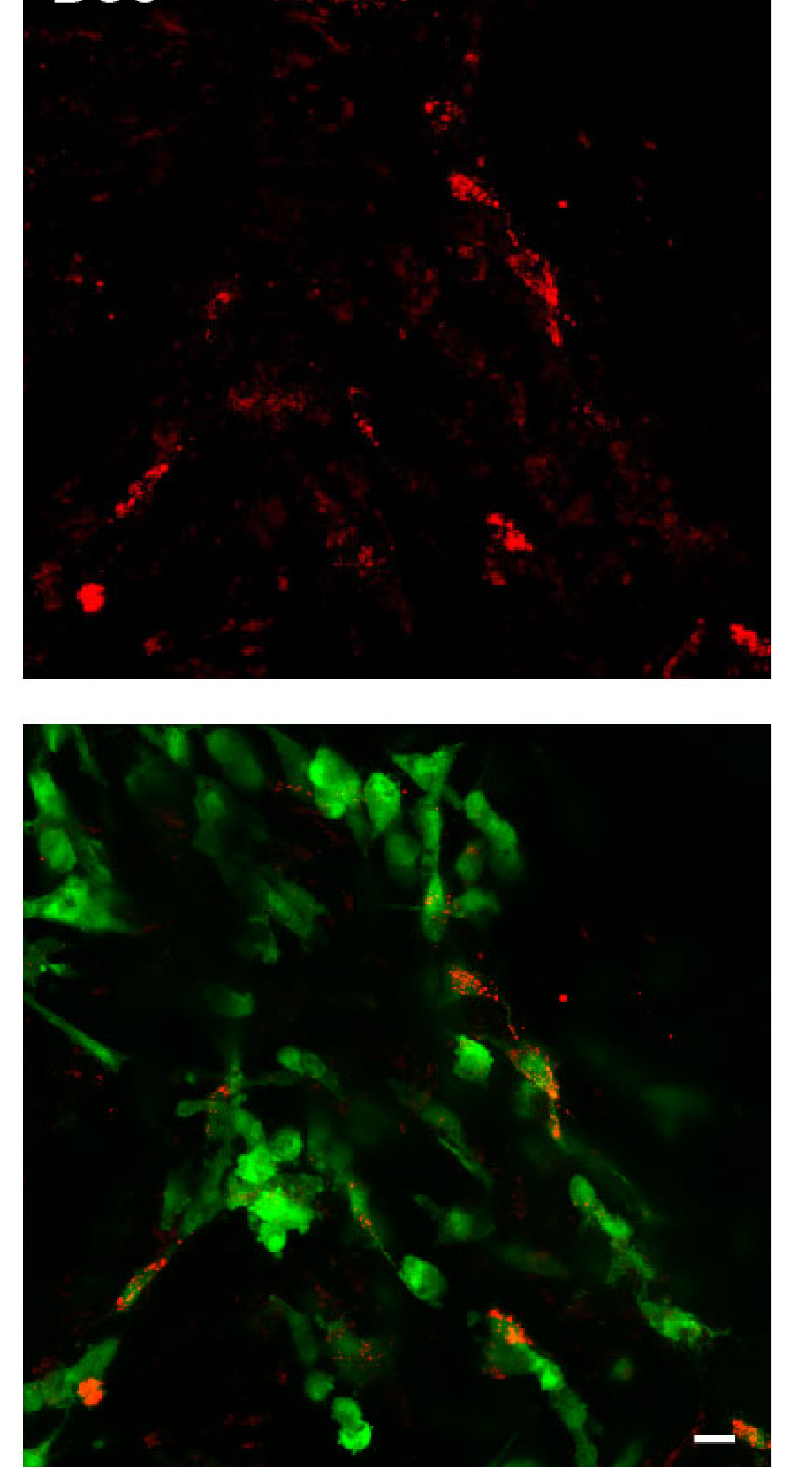


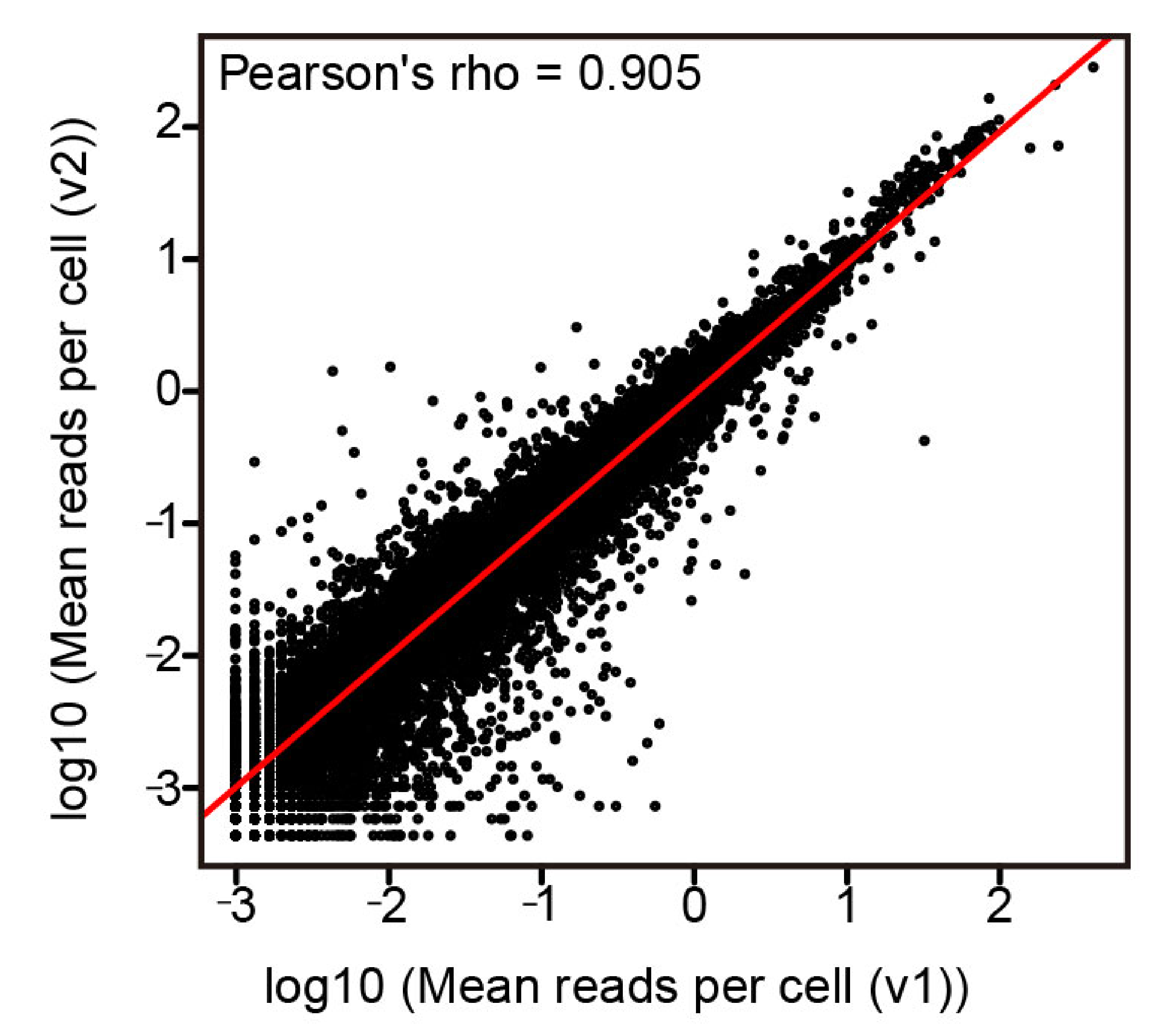

B

FB1 $\bigcirc \mathrm{PC} \bigcirc \mathrm{MKI} 67 \bigcirc \mathrm{EC} \bigcirc \mathrm{SMC} \bigcirc \mathrm{IM} \odot \mathrm{FB} 2 \bigcirc \mathrm{MG}$

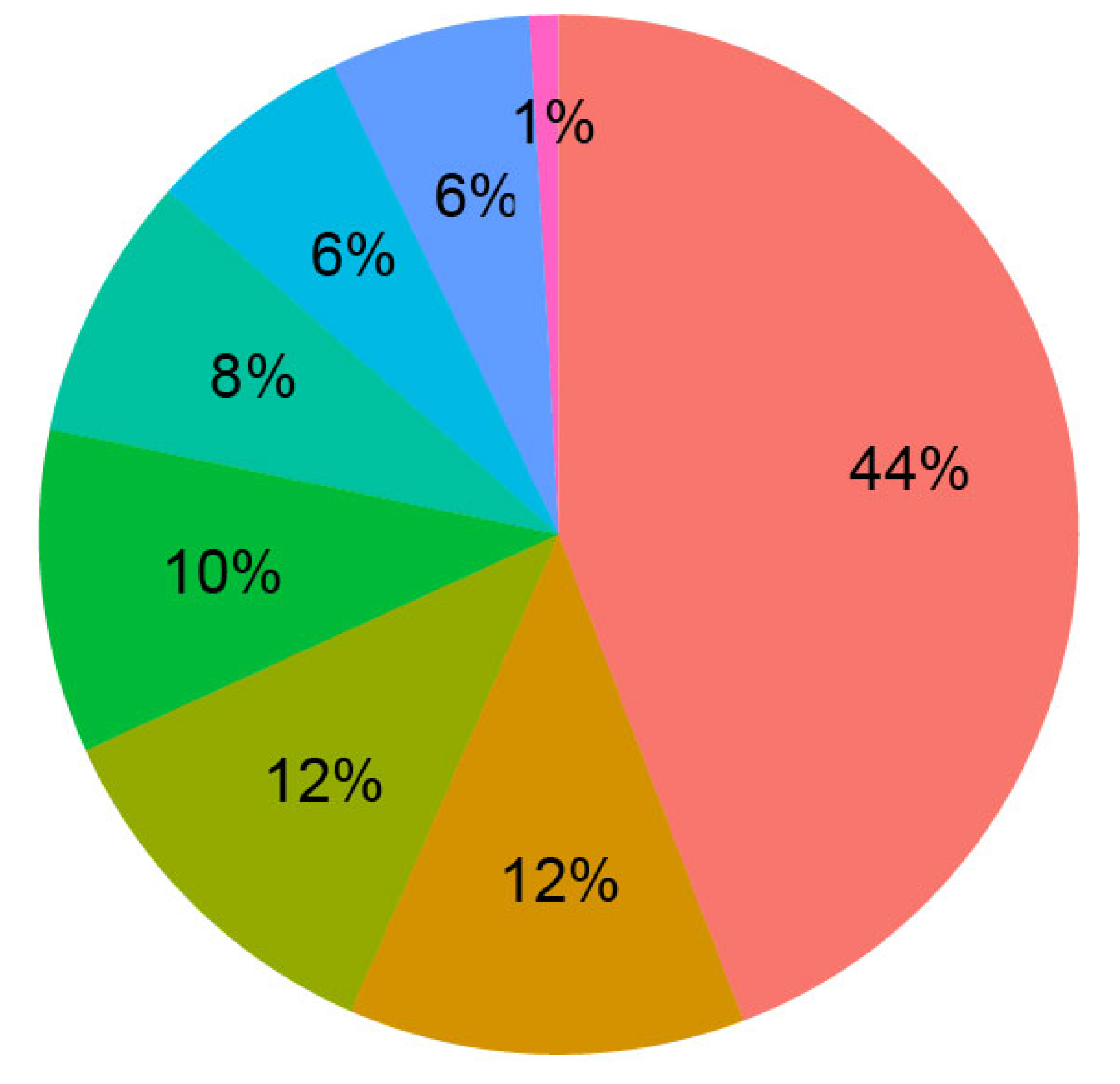

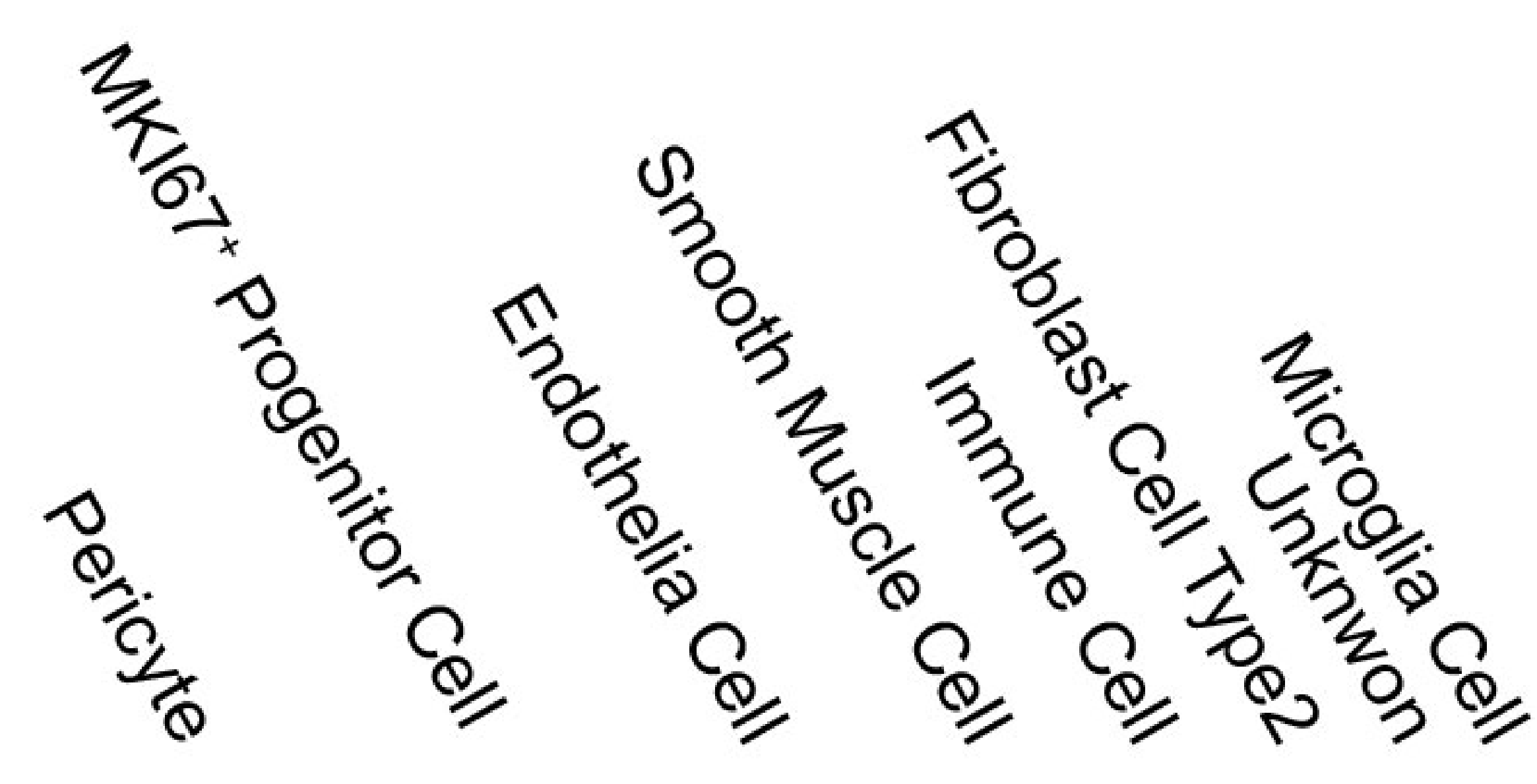

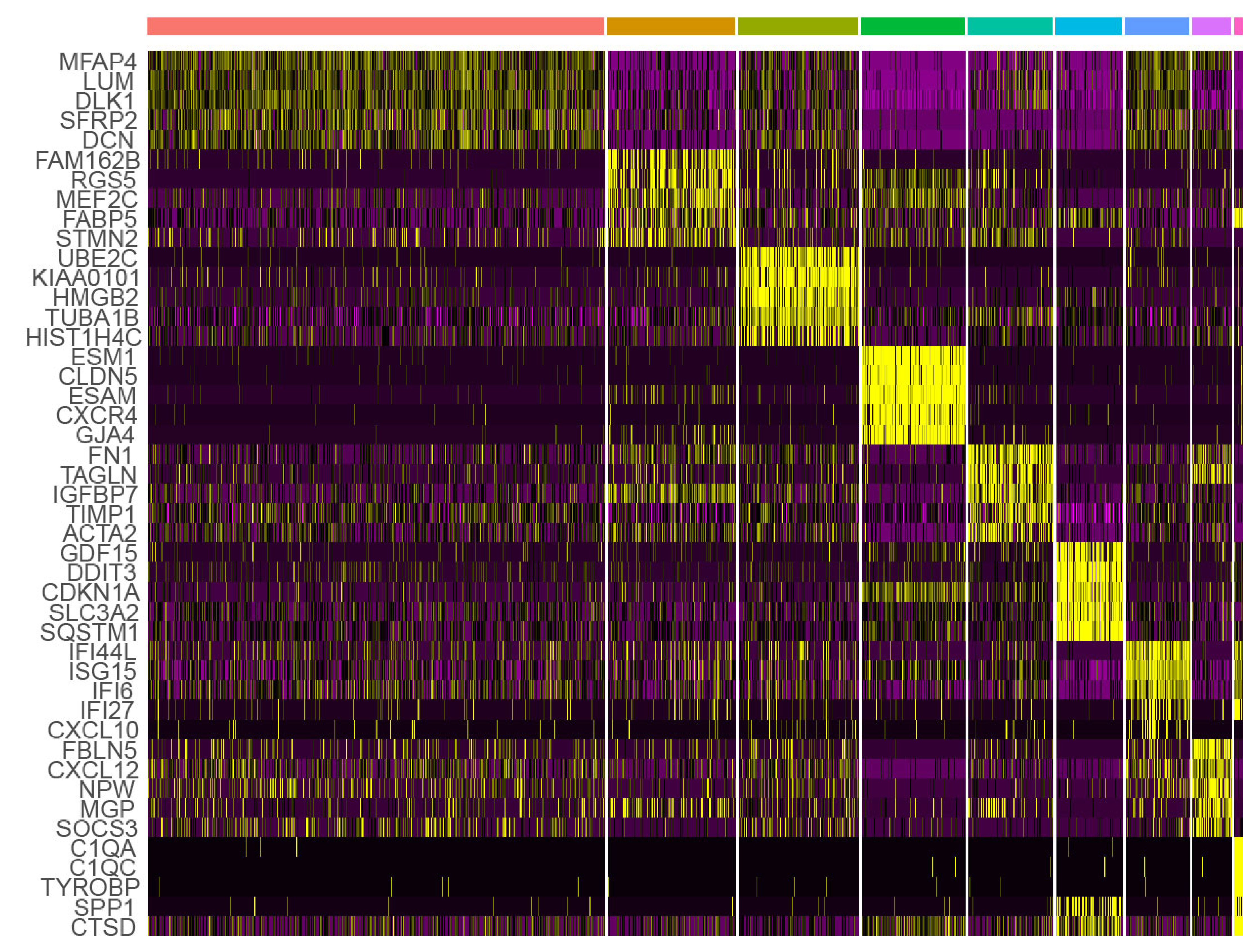

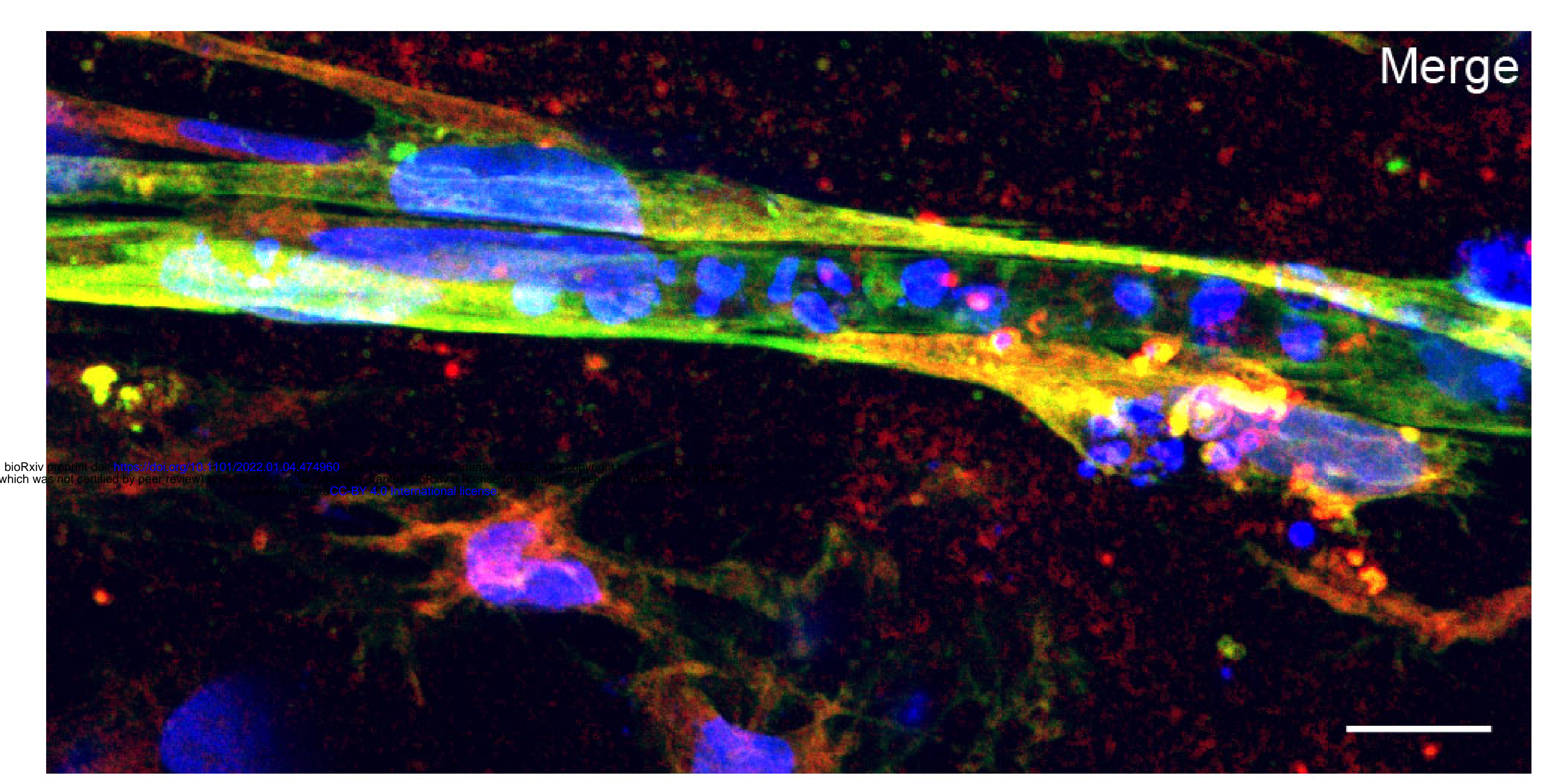
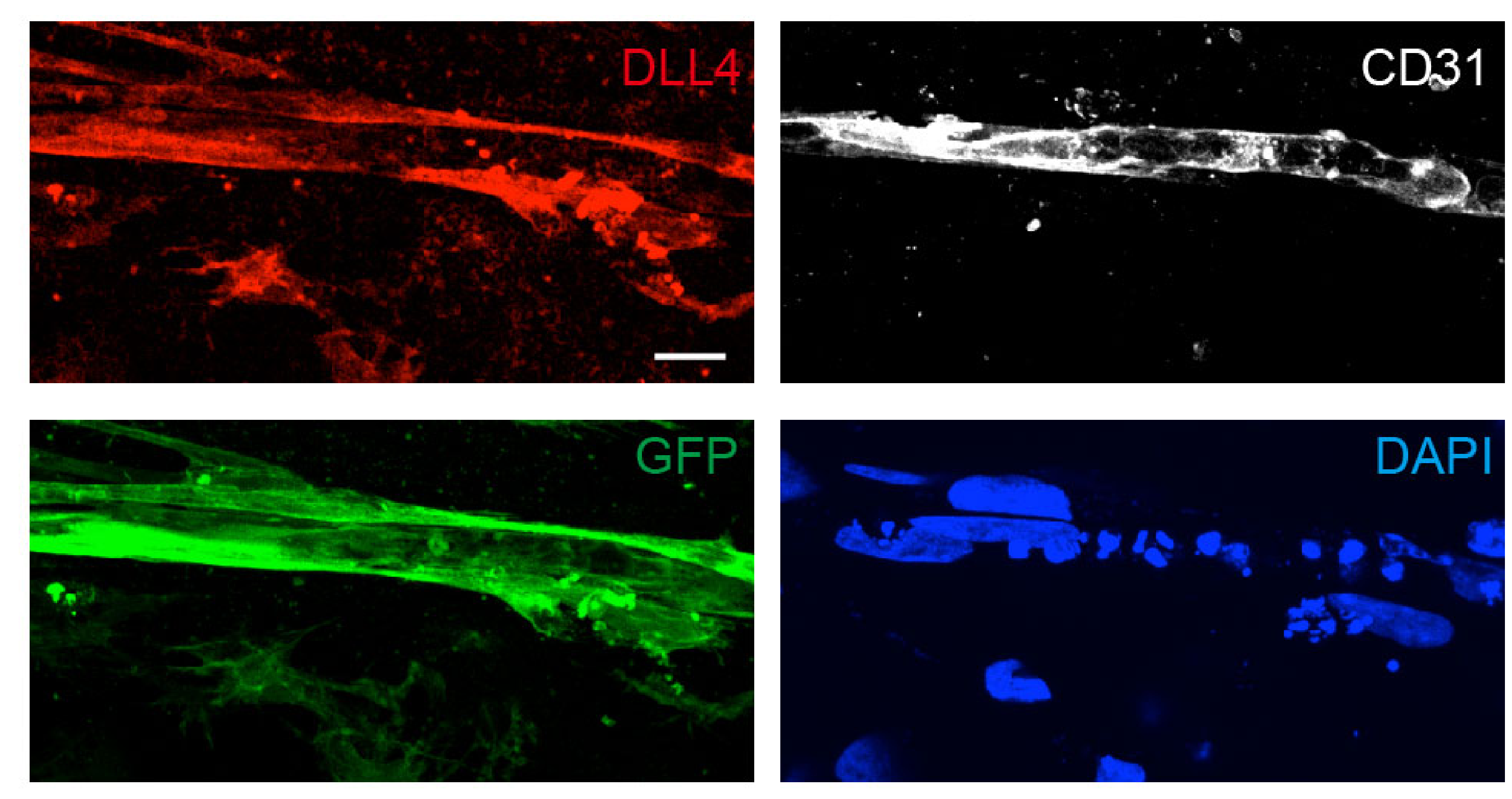

E
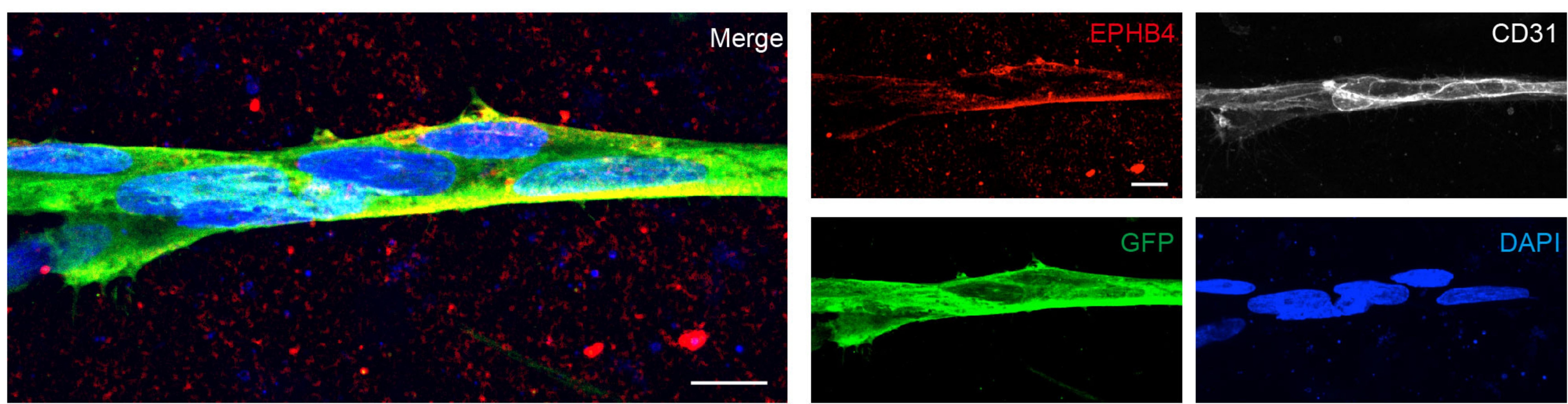

DAPI

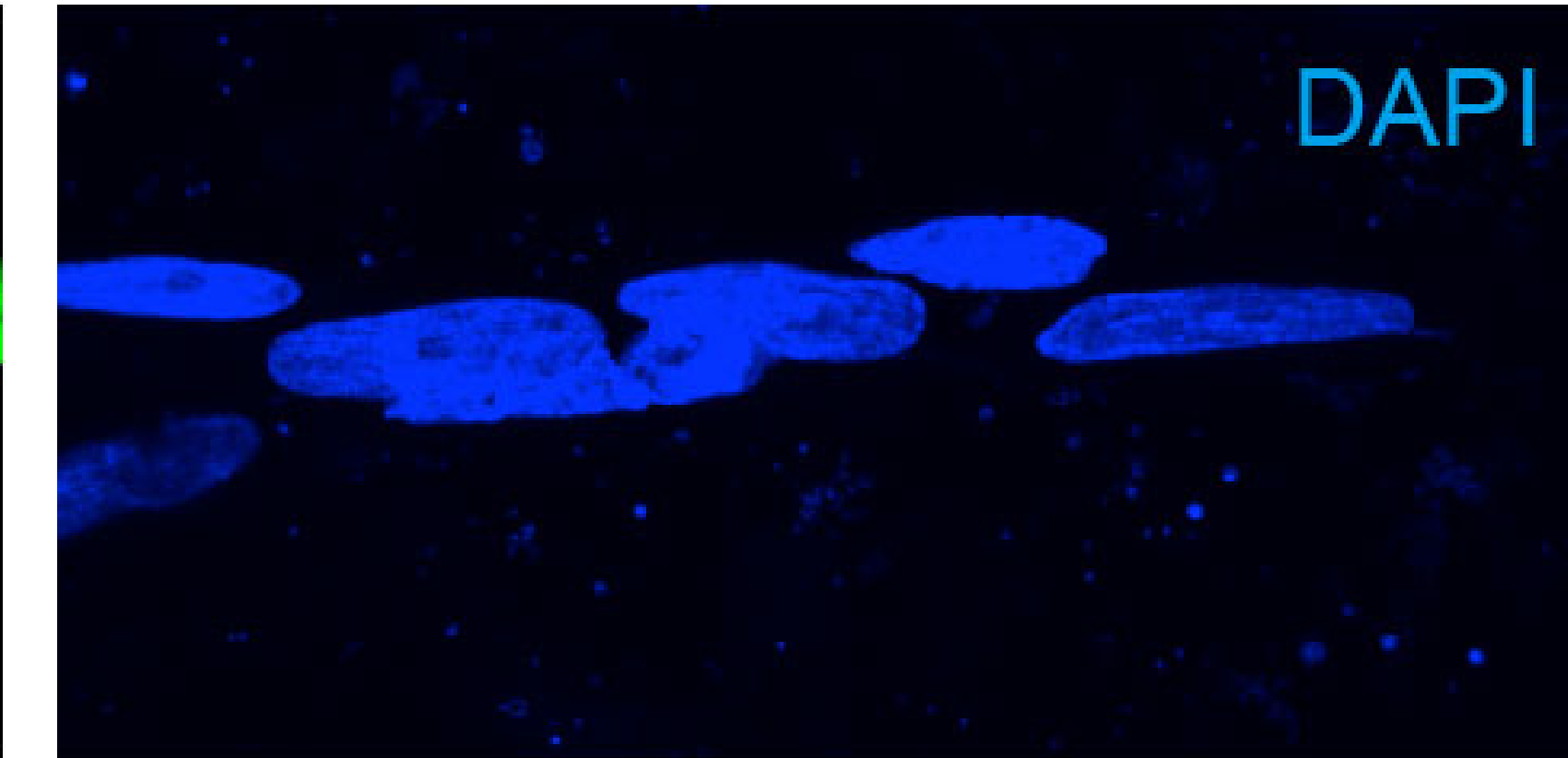


Pseudotime

0102030

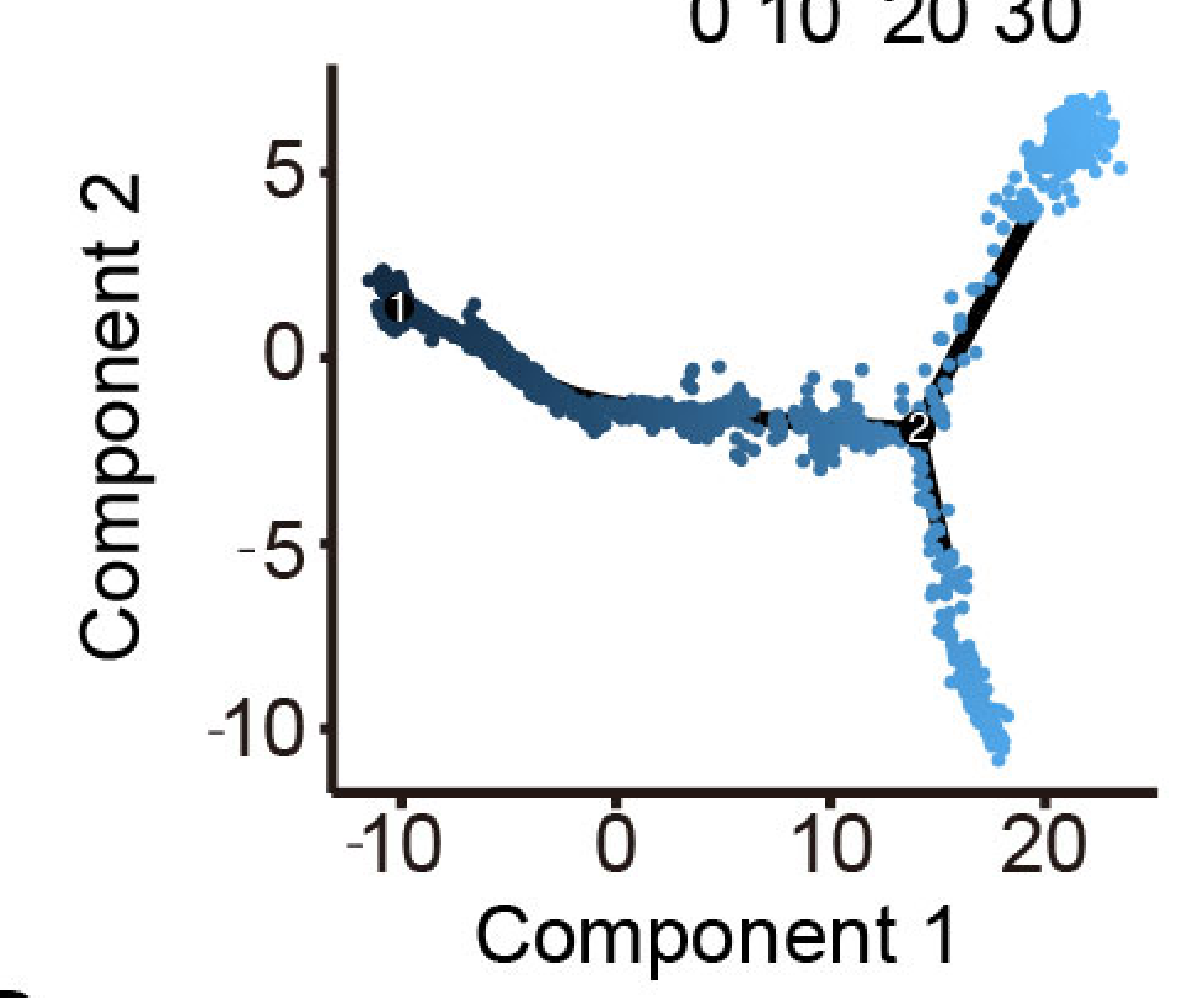

B

seurat clusters ${ }^{\circ} \mathrm{FB} 1 \cdot \mathrm{MKI} 67 \cdot \mathrm{SMC}$

${ }^{\circ} \mathrm{EC} \cdot \mathrm{FB} 2$ : $\cdot \mathrm{UN}$

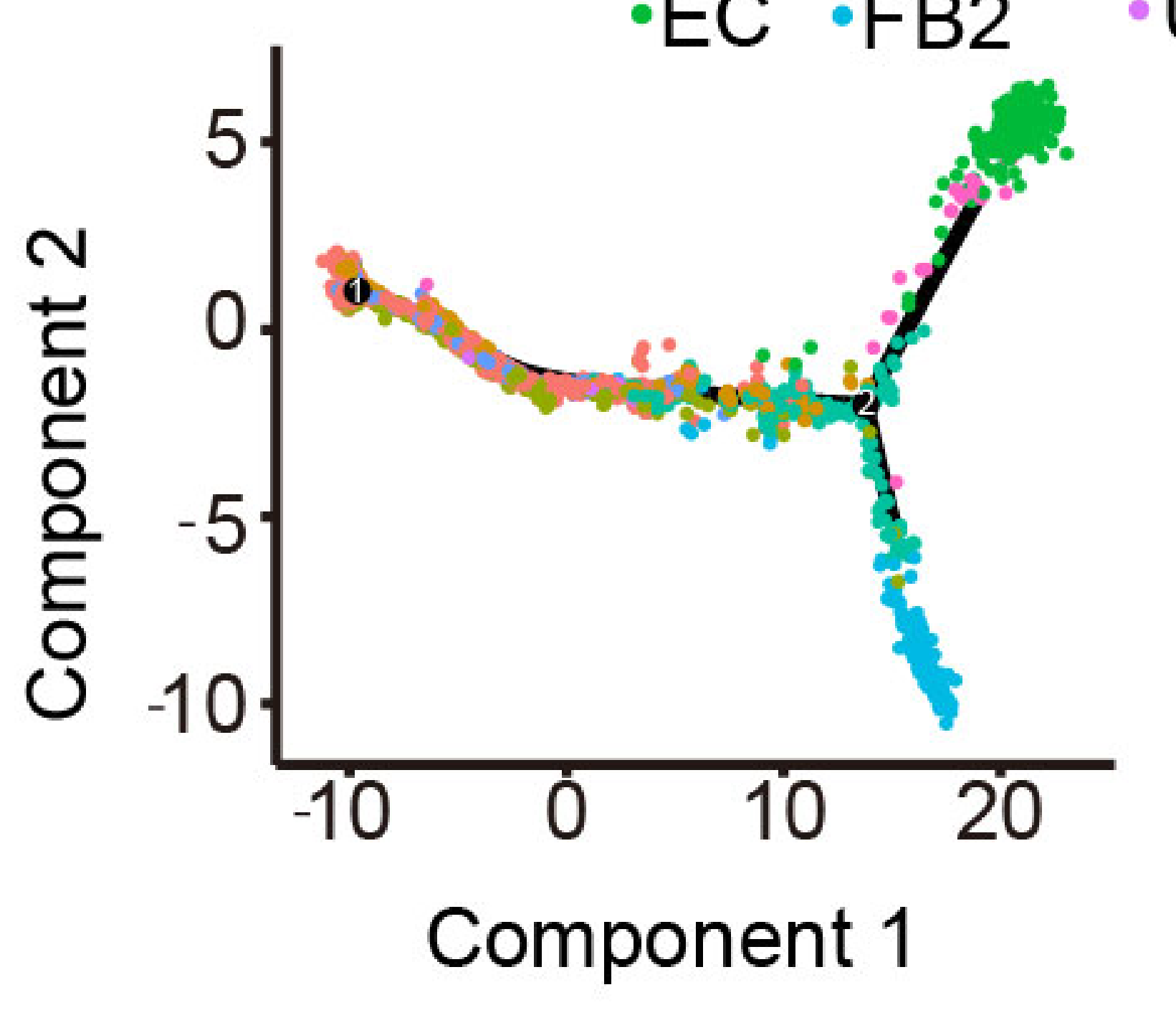

E

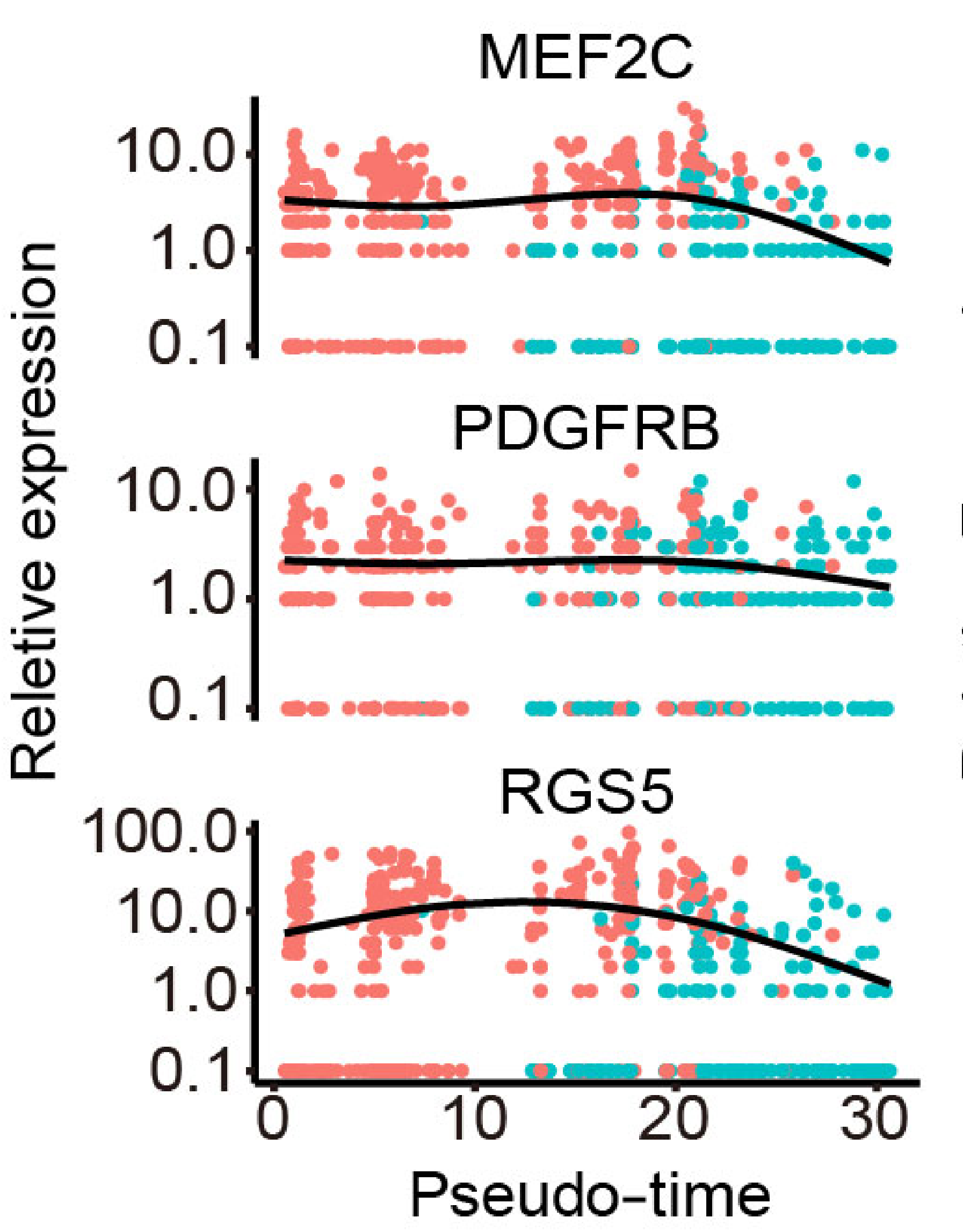

C

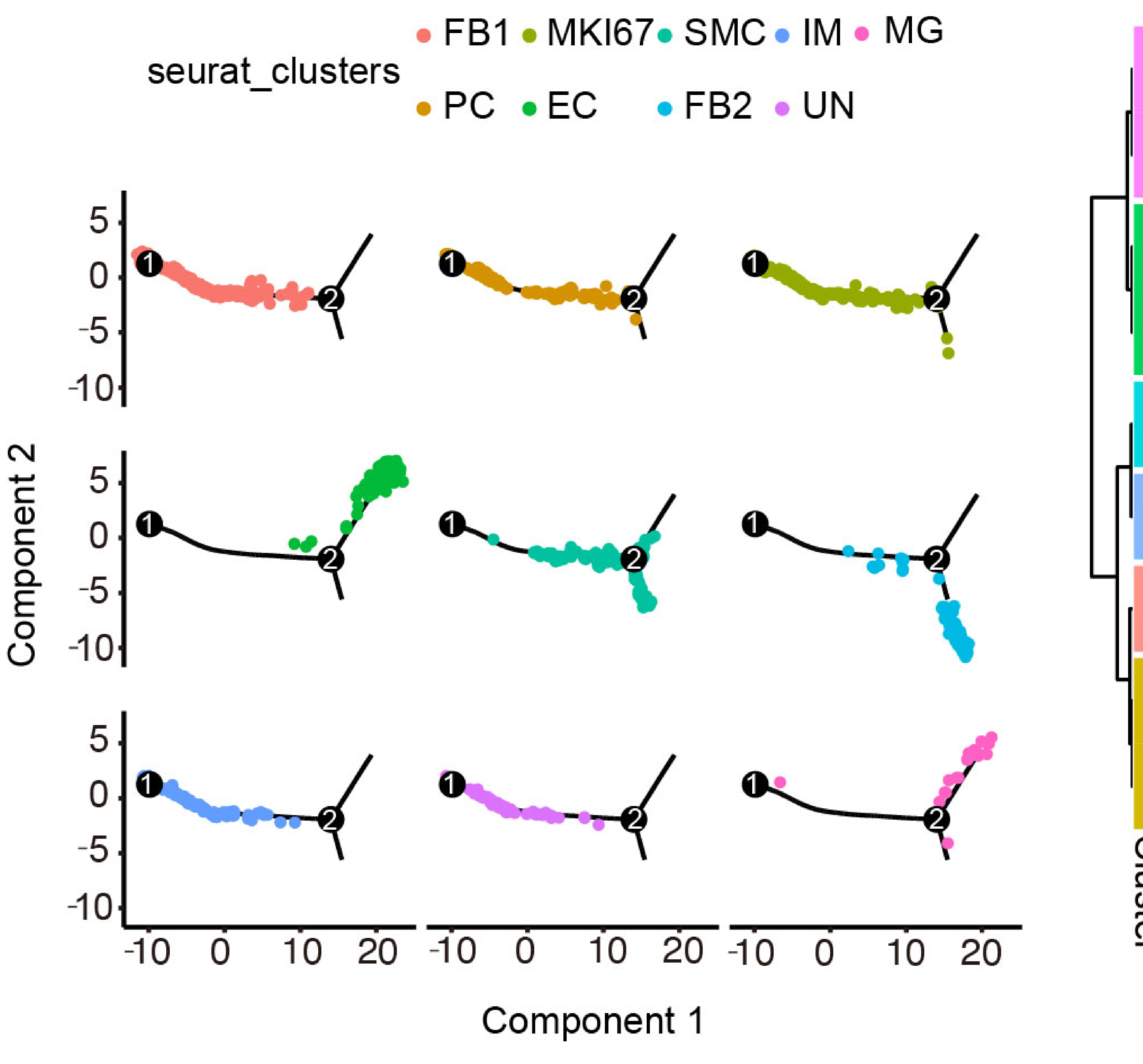

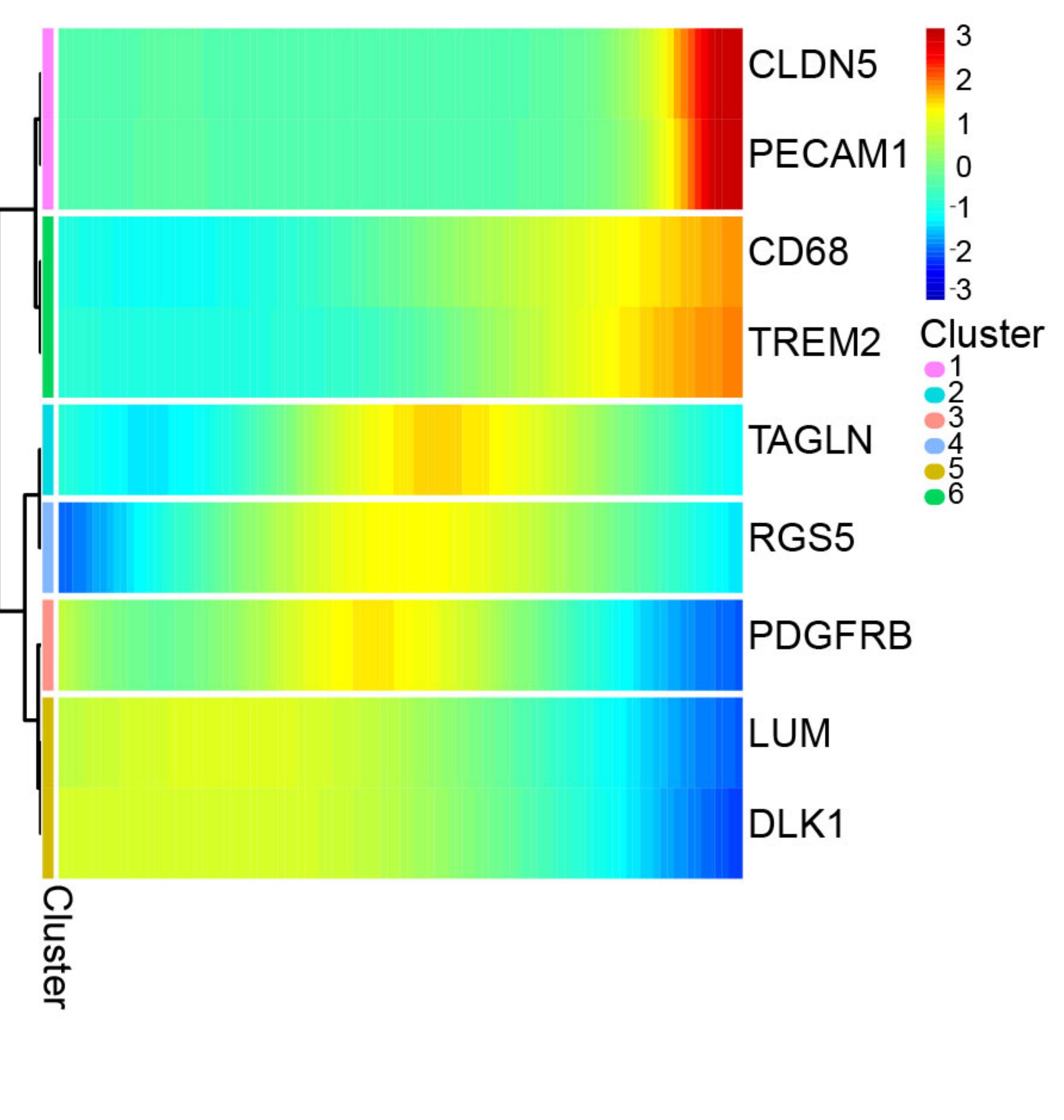

F $\quad$ G

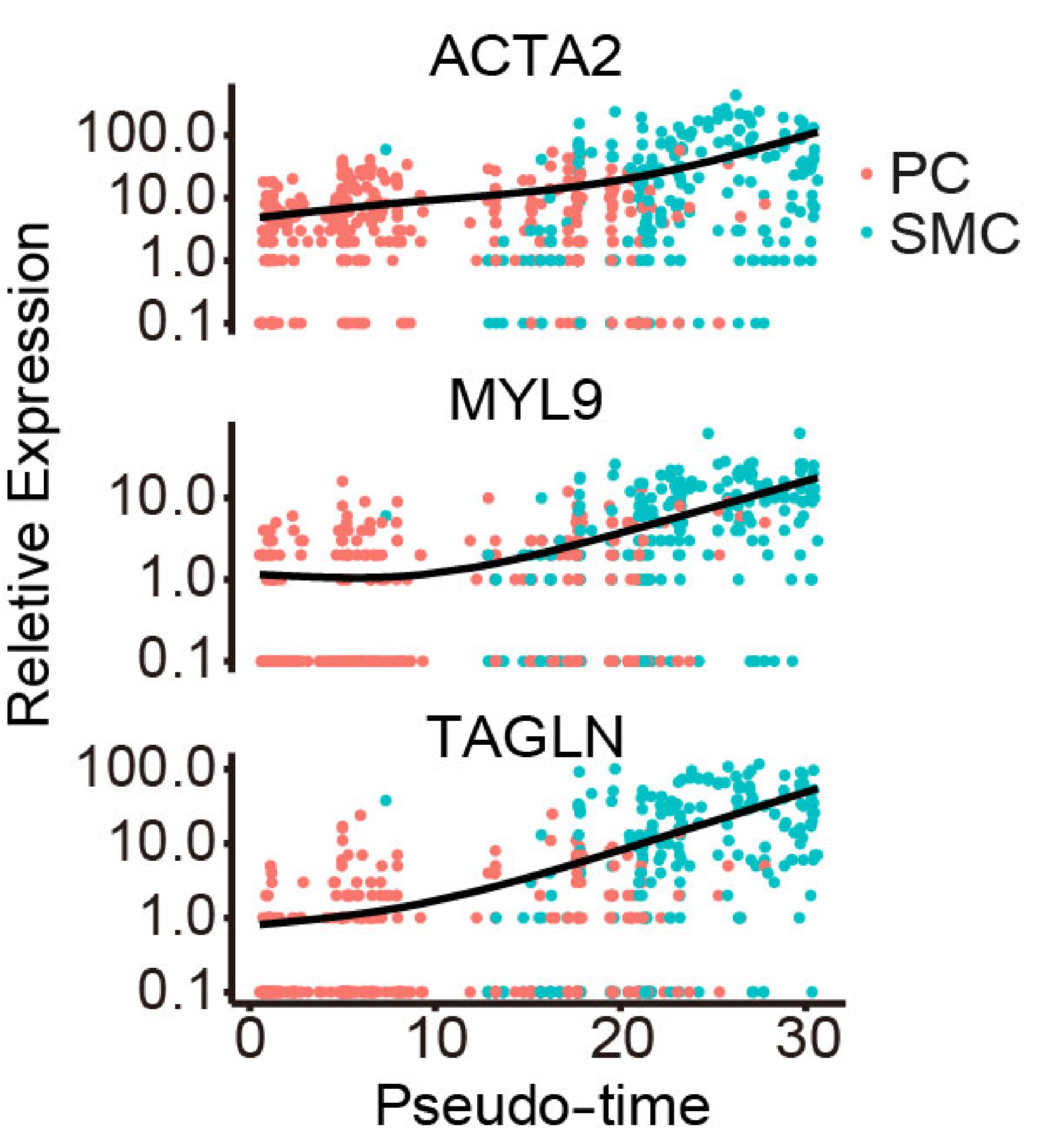

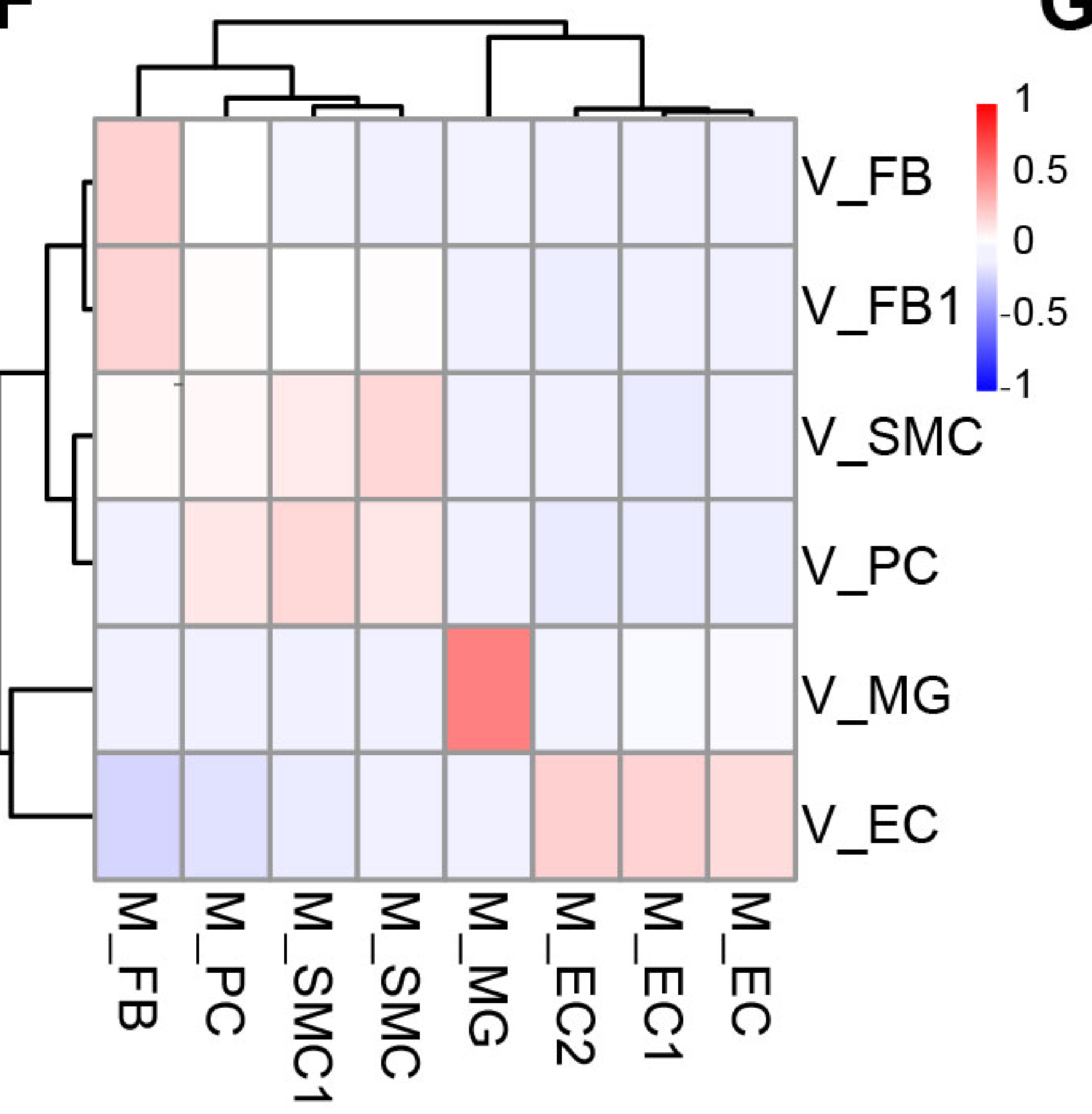

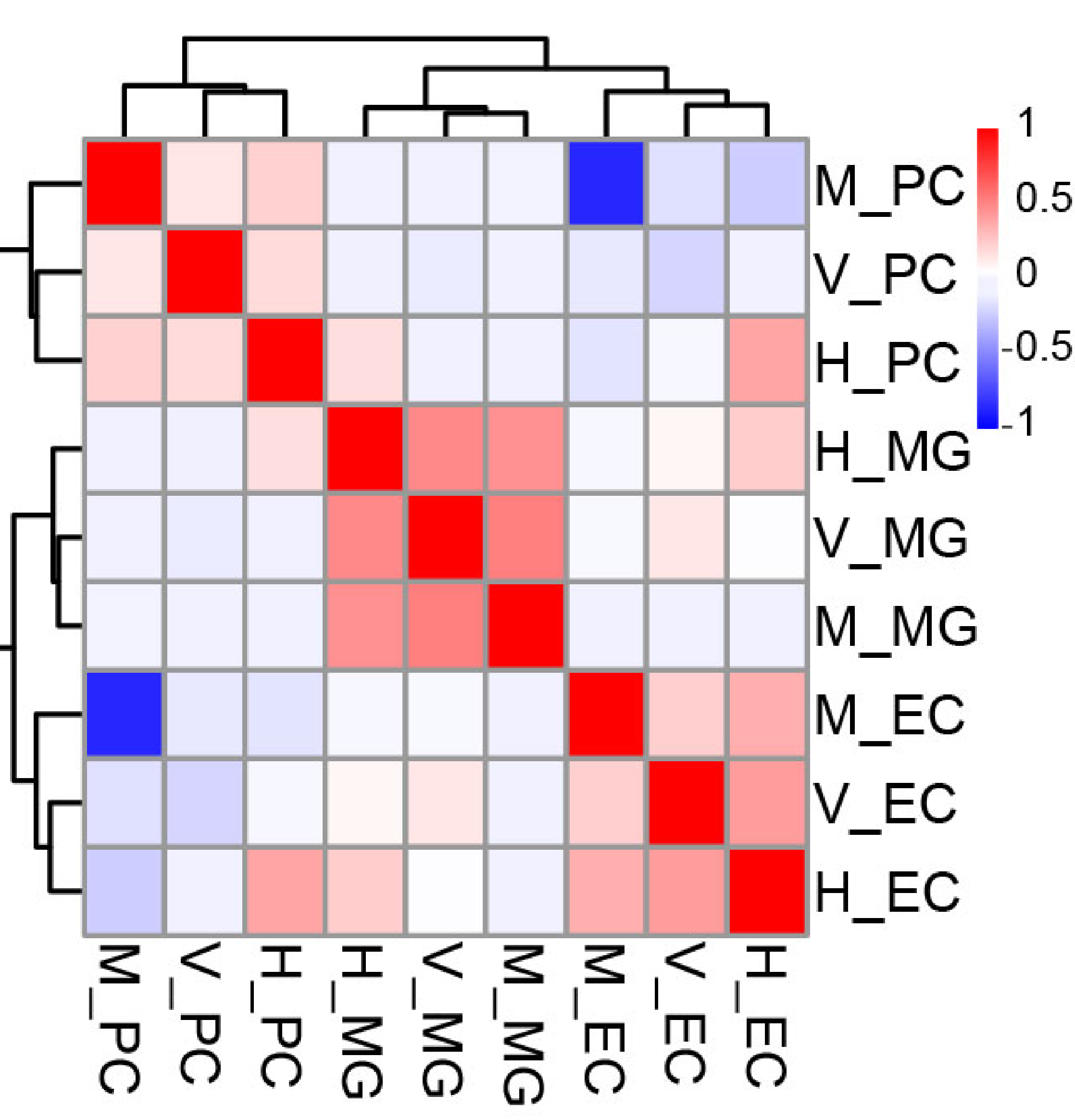

H
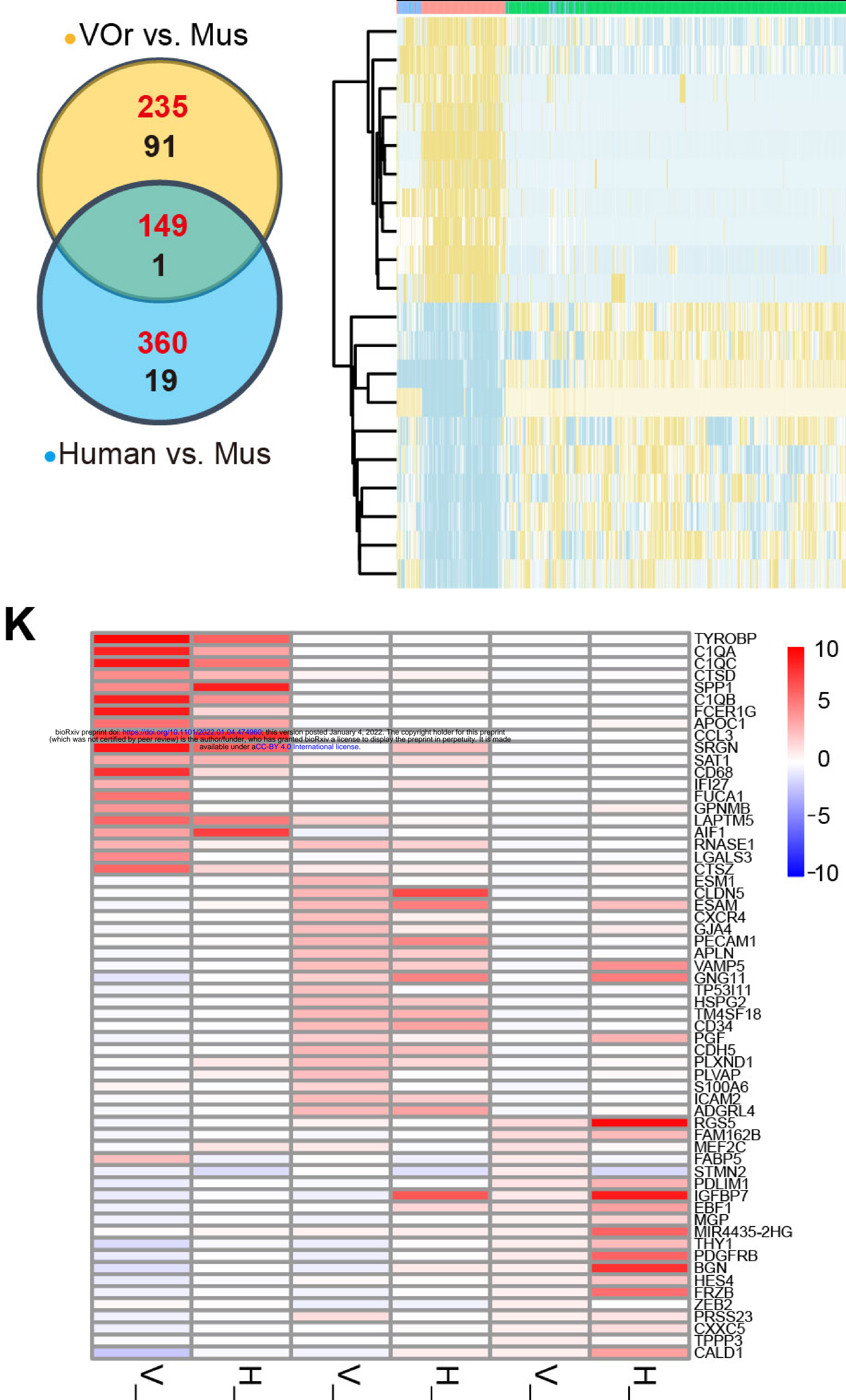

J

CellType
HSPG2 ARHGAP29
APLN

TMEM255B -0.5

ESM1

MCAM

PERP

GJA4

CXCR4

RPS5

LY6E

COL3A1

RPL8

RPL13A

RPL32

RPL10A $\begin{array}{ll}\text { HSPG2 } & 0.5\end{array}$

regulation of vasculature development regulation of angiogenesis ameboidal-type cell migration epithelial cell migration epithelium migration tissue migration endothelial cell migration. cell-substrate adhesion. CellType HUM VOR vasculature development. cell junction assembly. cell junction organization. blood vessel endothelial cell migration. positive regulation of angiogenesis. endothelium development. vasculogenesis.

$$
0.075 \cdot 100 \cdot 120.150^{0} \cdot 17_{5}
$$

GO Terms:

p_value:

2.20E-24

neutrophil degranulation

$2.62 \mathrm{E}-24$

neutrophil activation involved in immune response

4.65E-24

neutrophil activation

$4.92 \mathrm{E}-24$

neutrophil mediated immunity

1.49E-09

epithelial cell proliferation

1.53E-08

regulation of vasculature development

1.14E-13

endothelium development

4.66E-08

regulation of angiogenesis

1.43E-08

mesenchyme development

$2.18 \mathrm{E}-05$

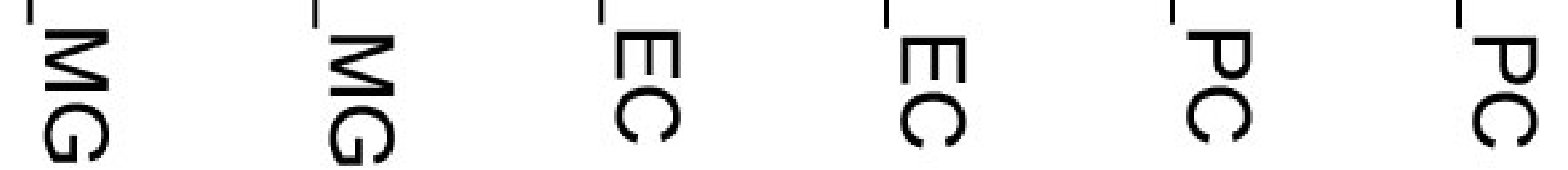




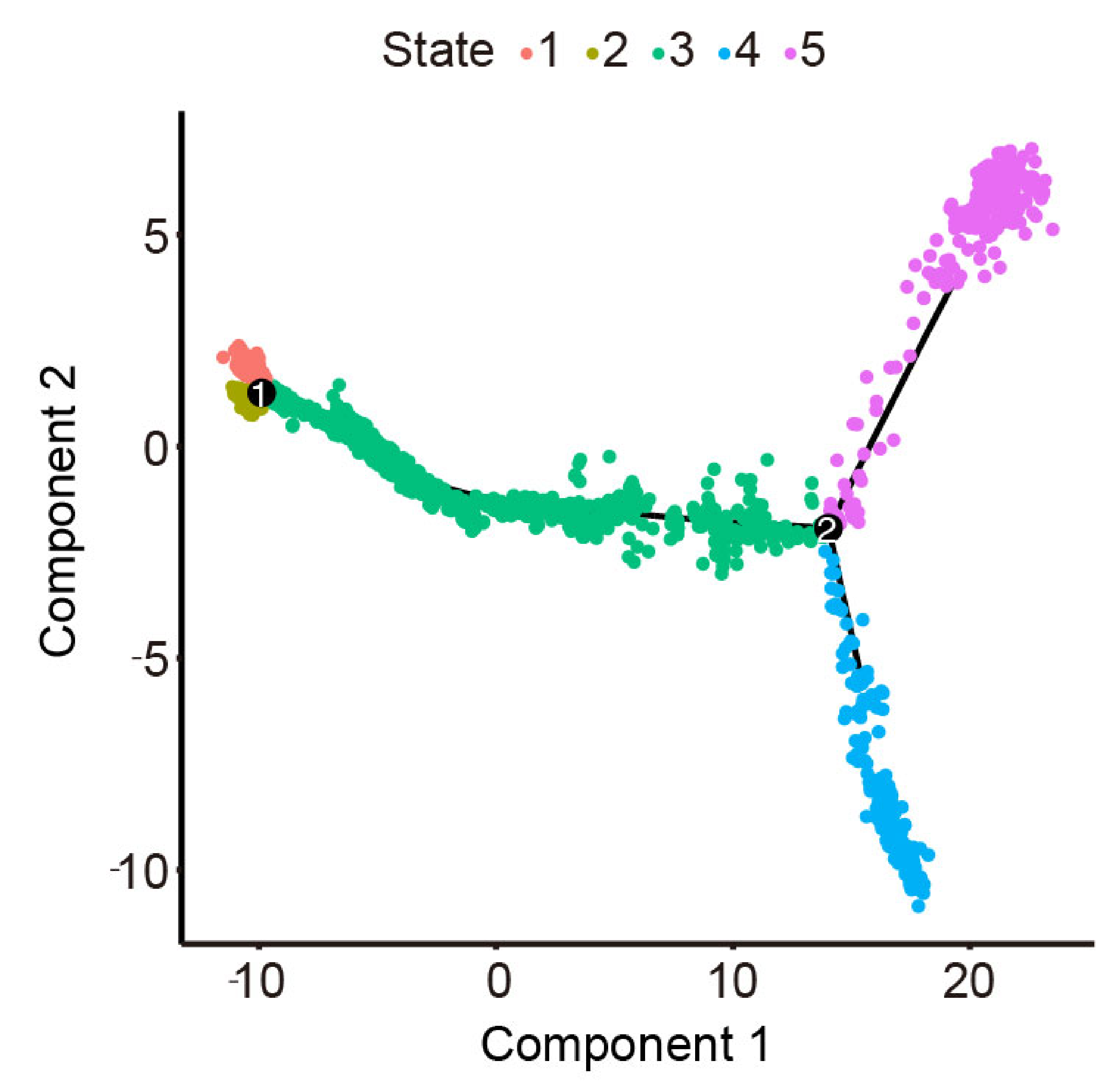

C

B

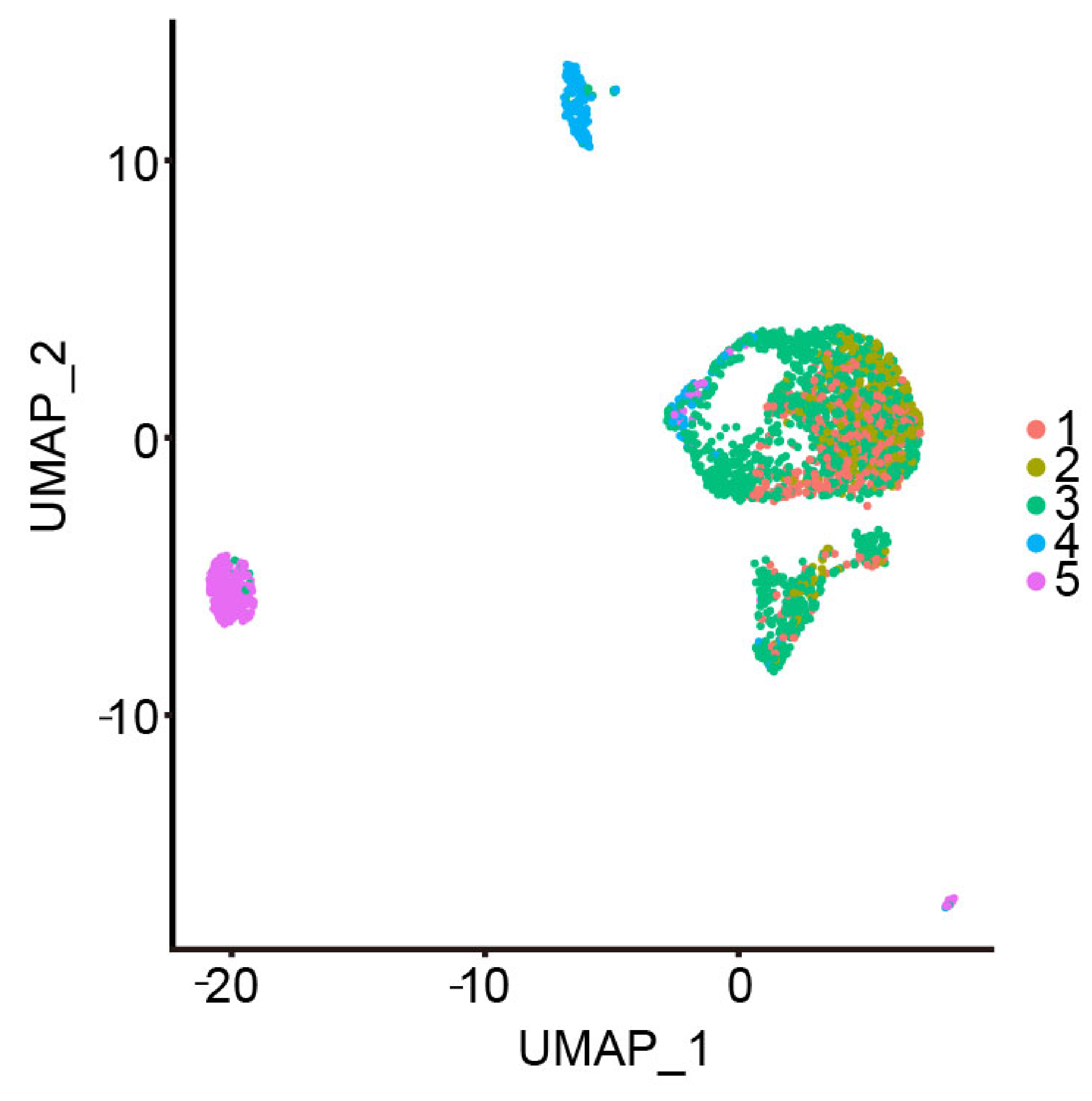

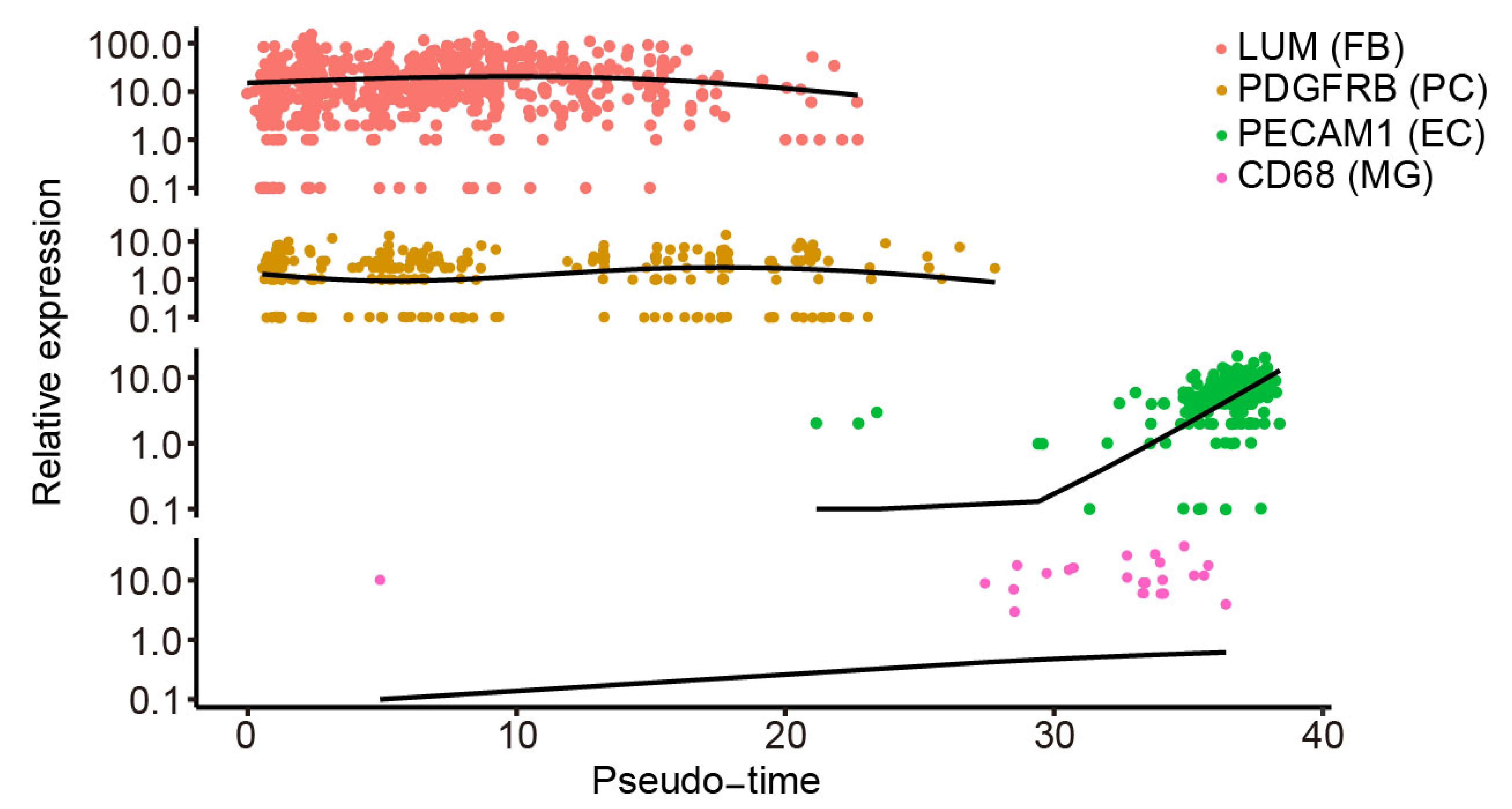

D

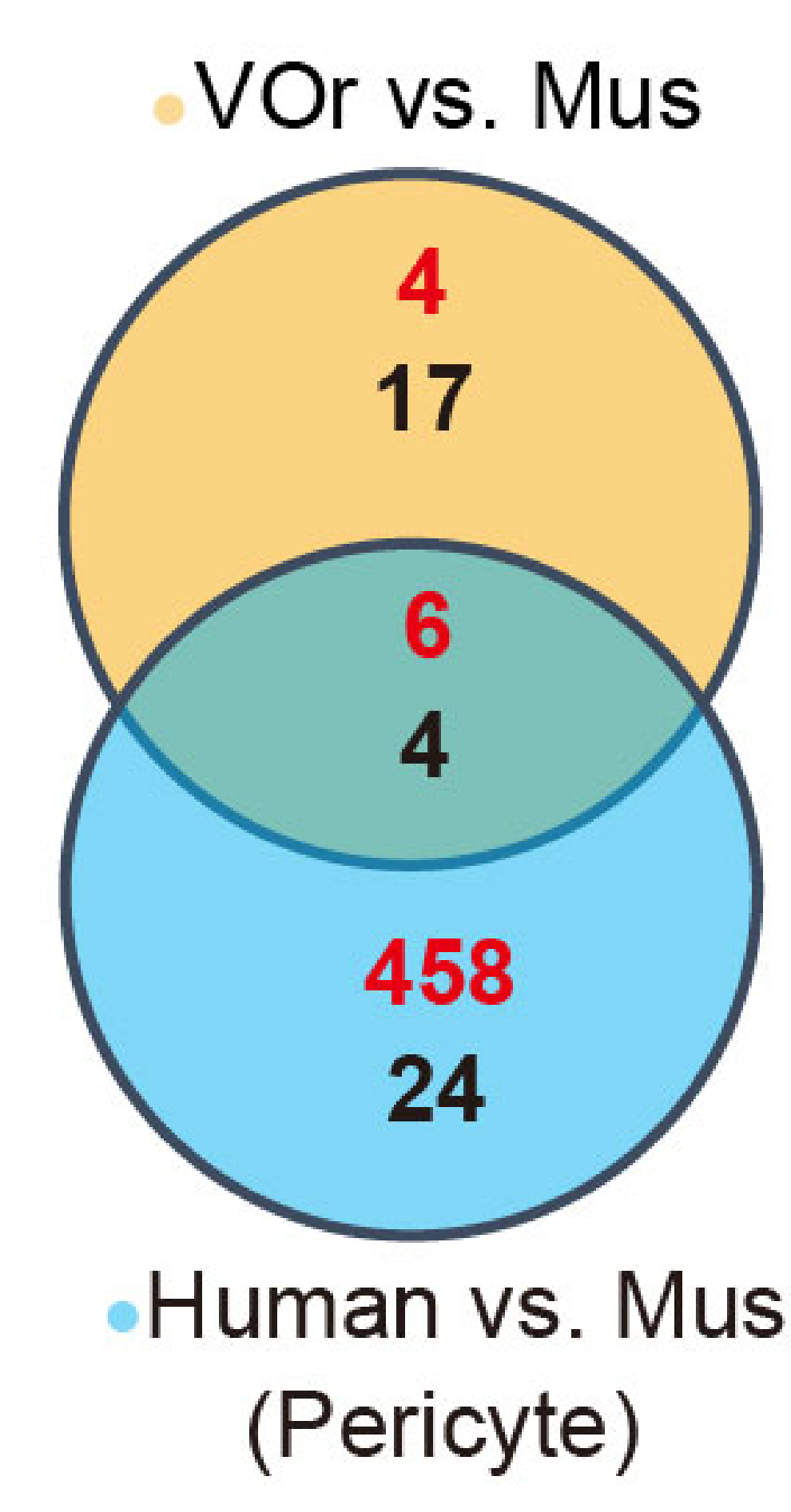

E

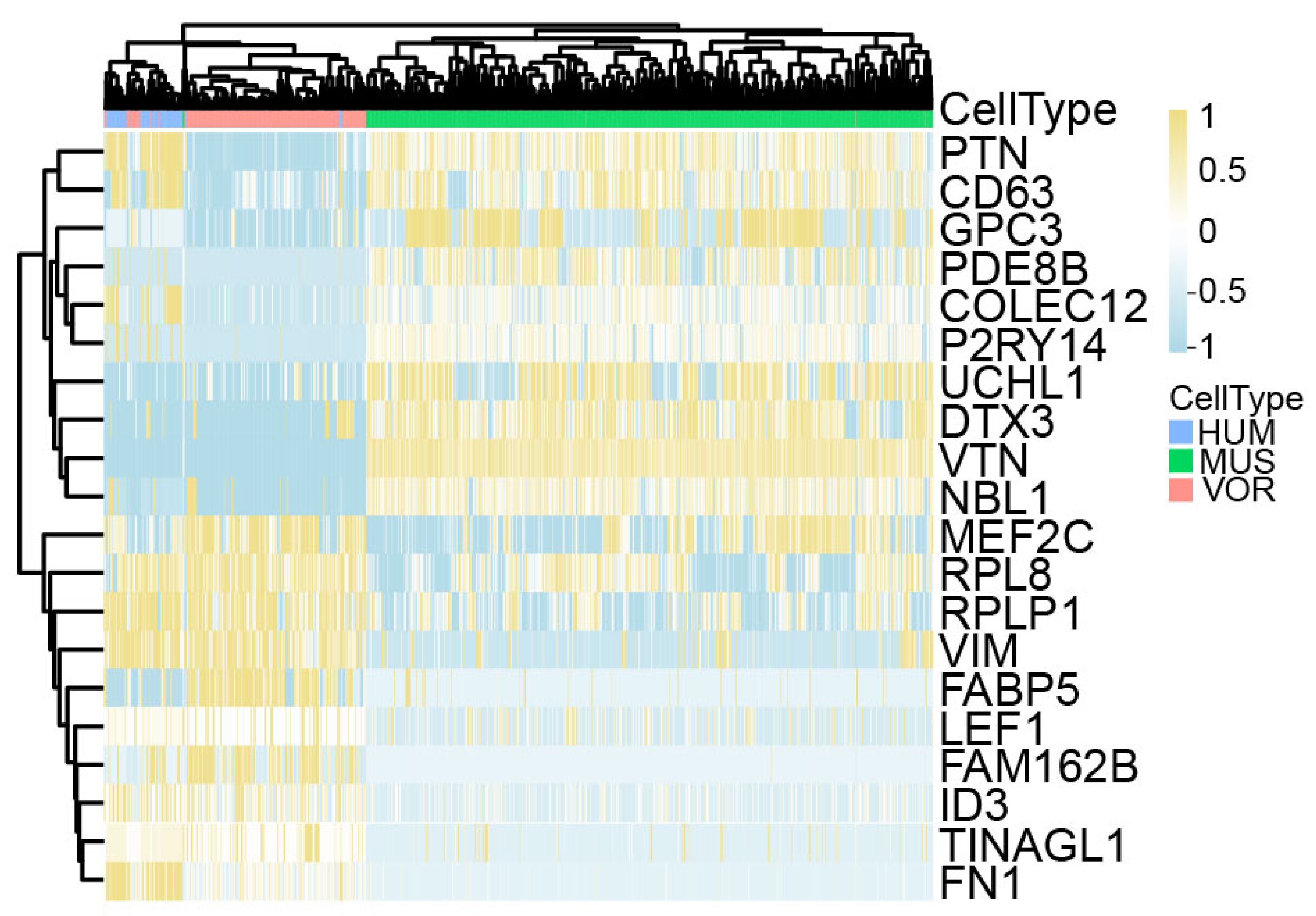

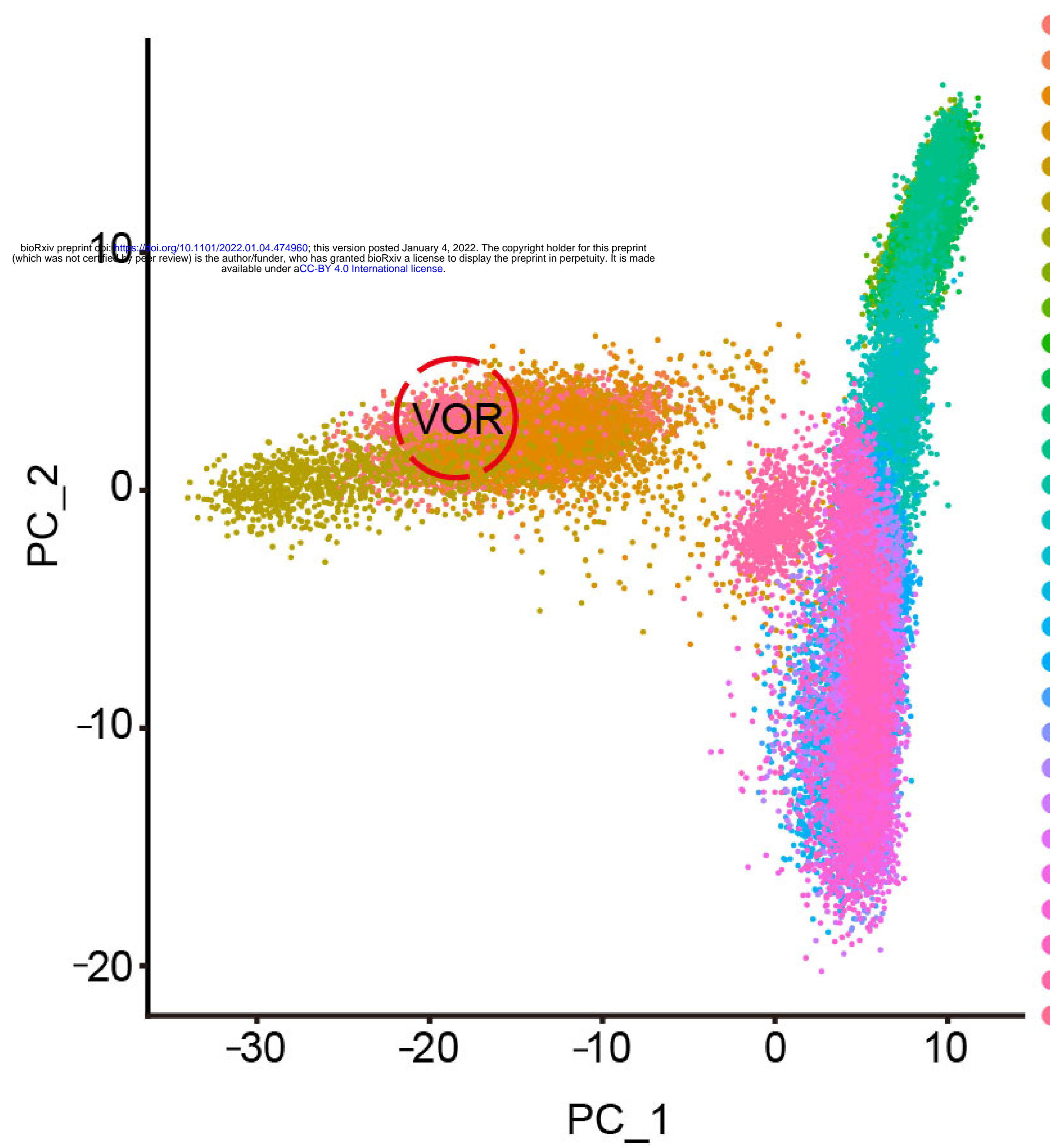

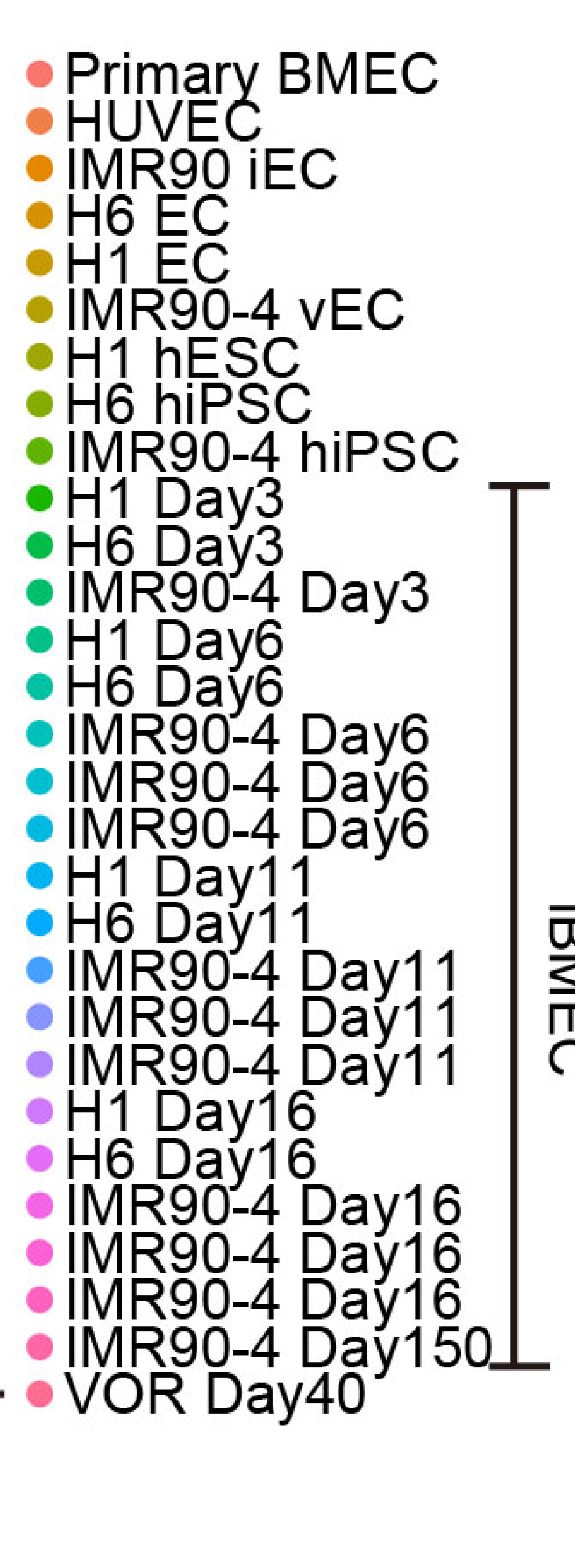

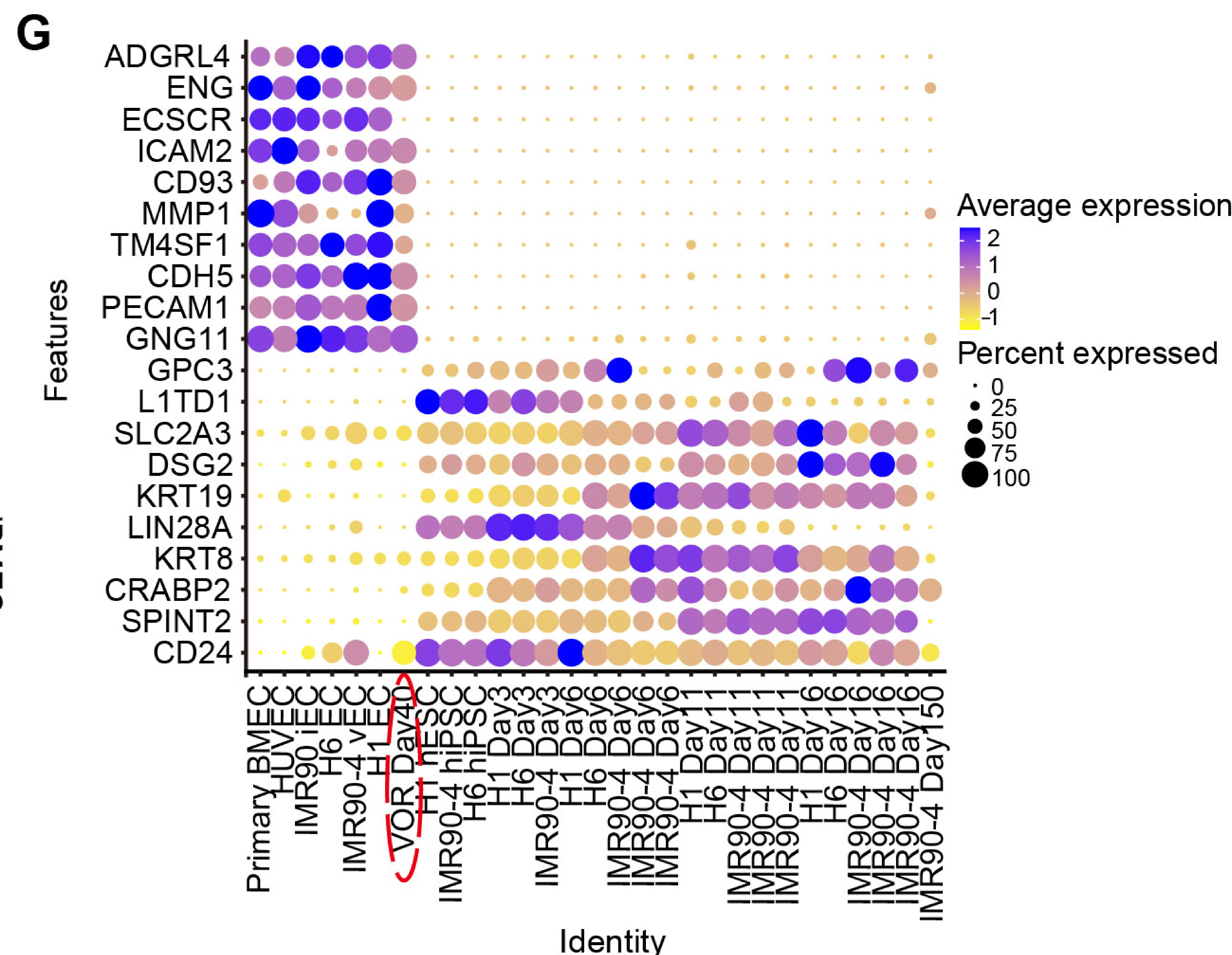




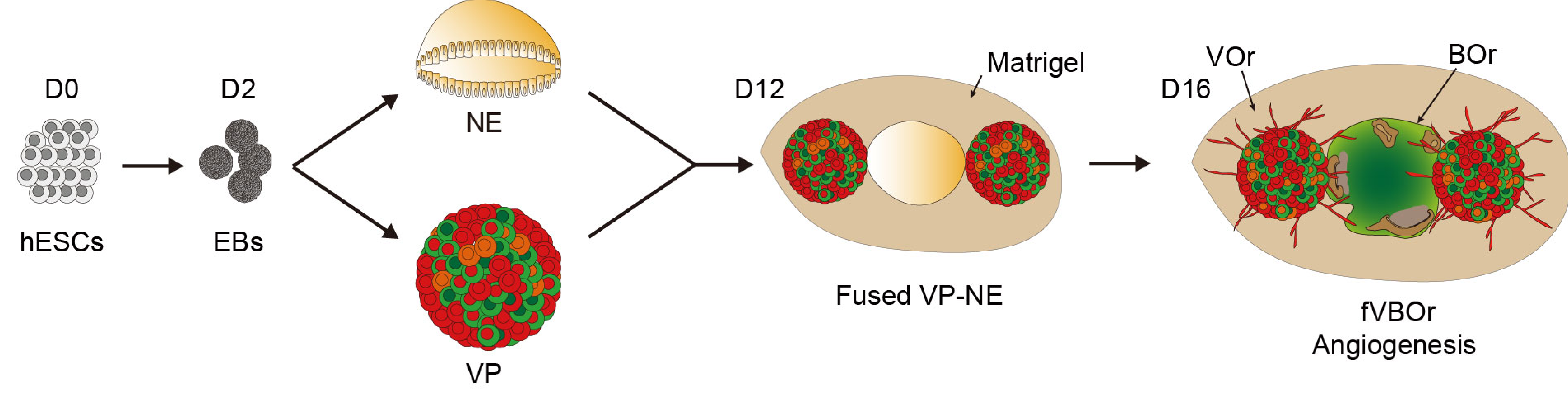

B
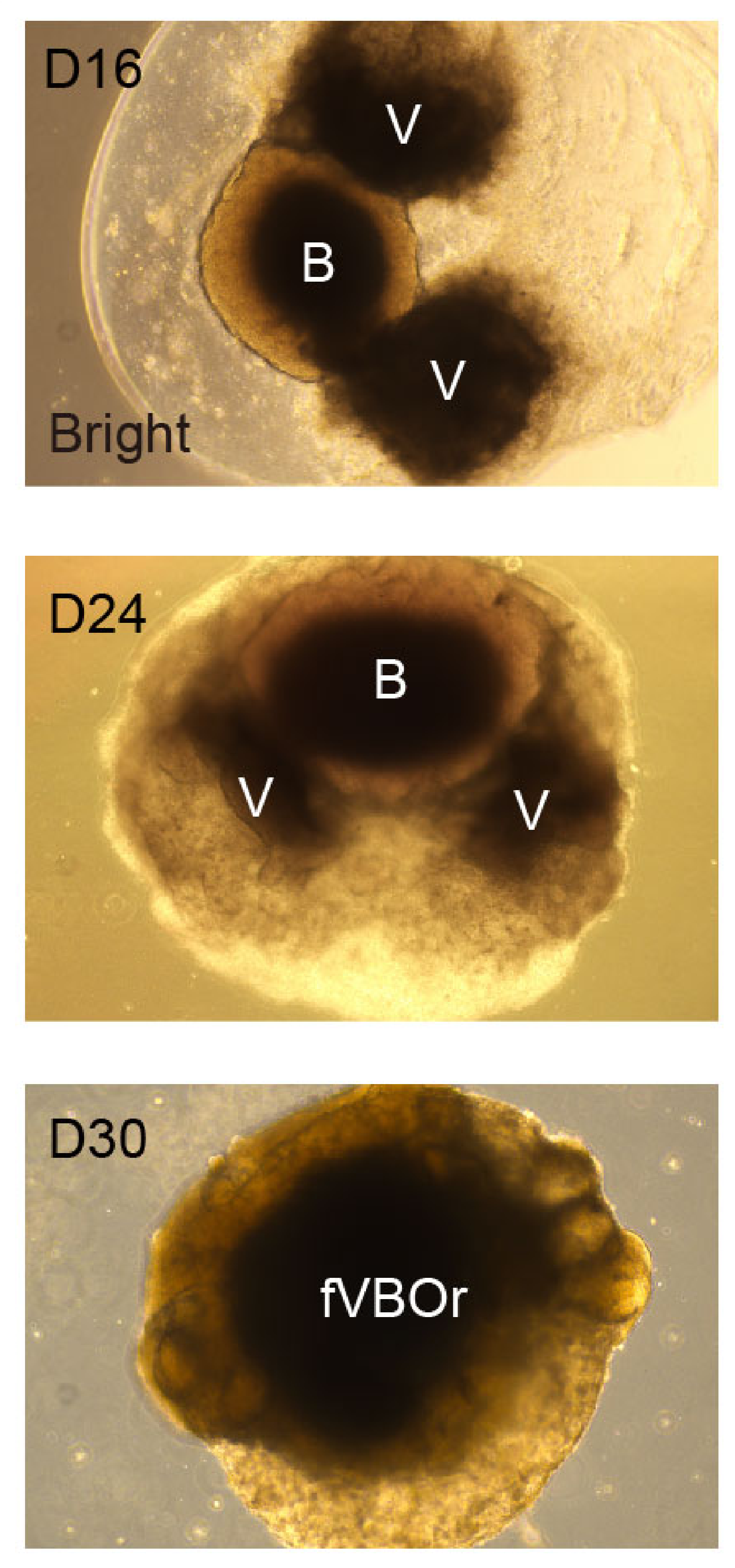

D$$
\text { (D) }
$$

\section{D40-fVBOr}

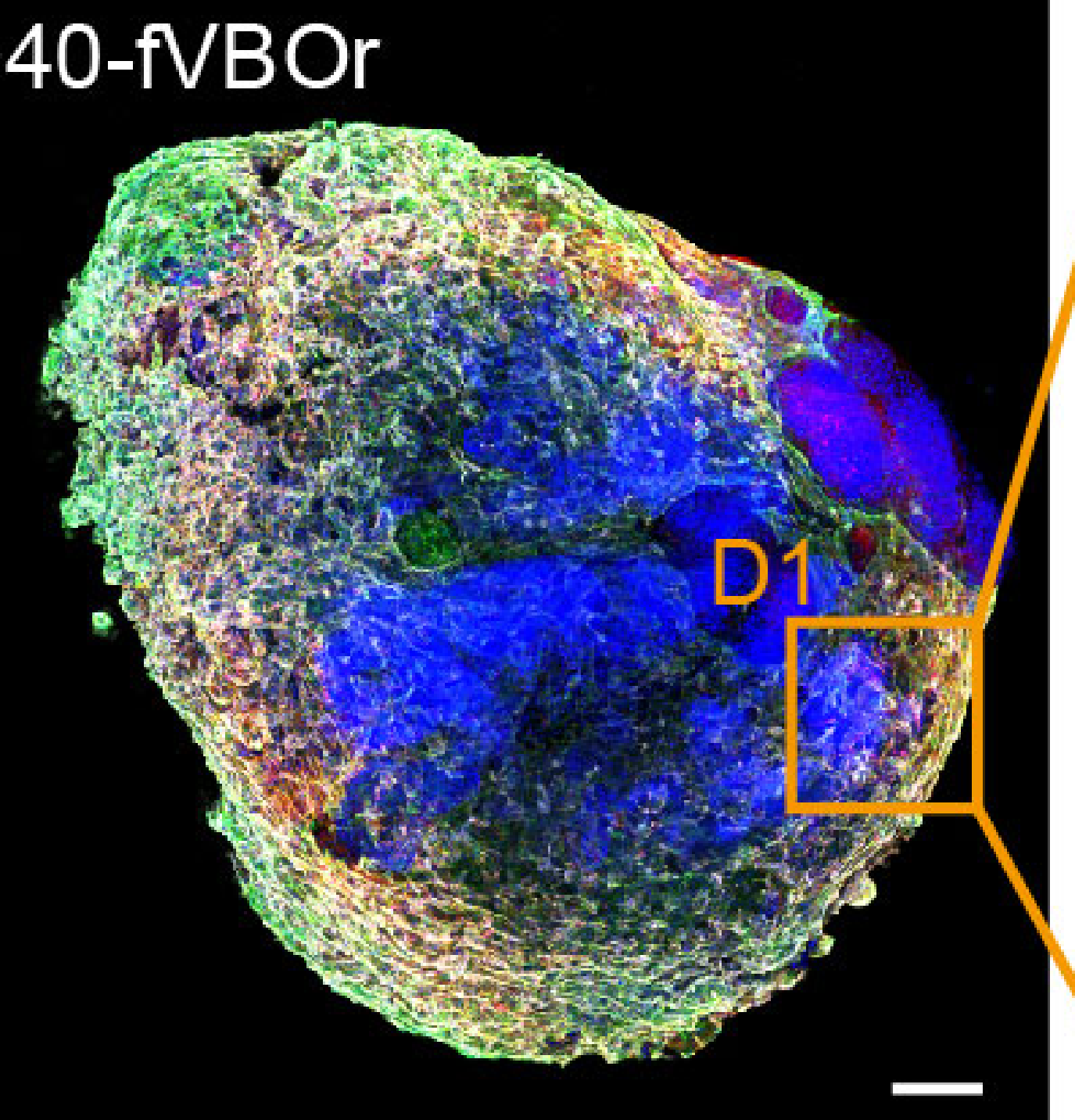

-
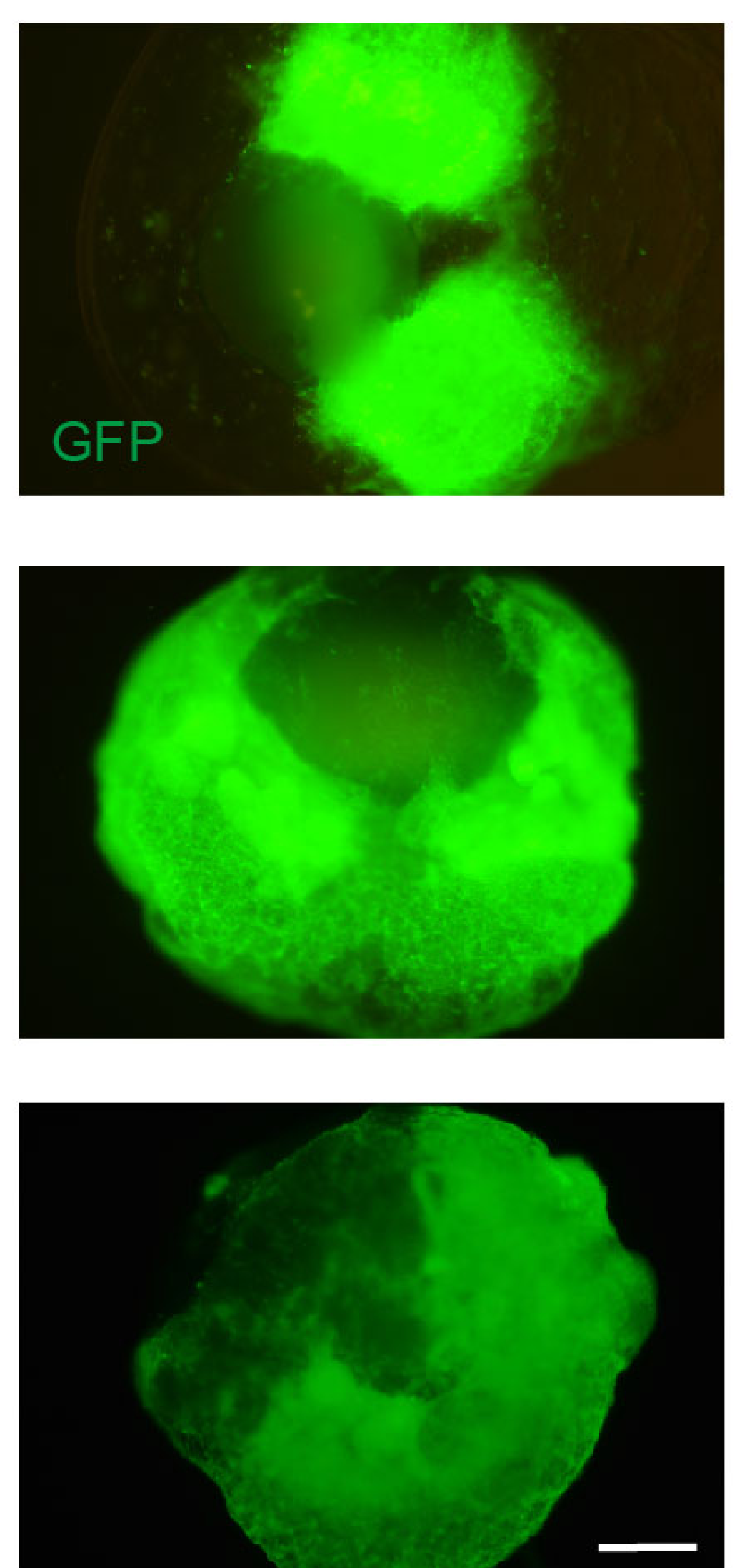

Dit

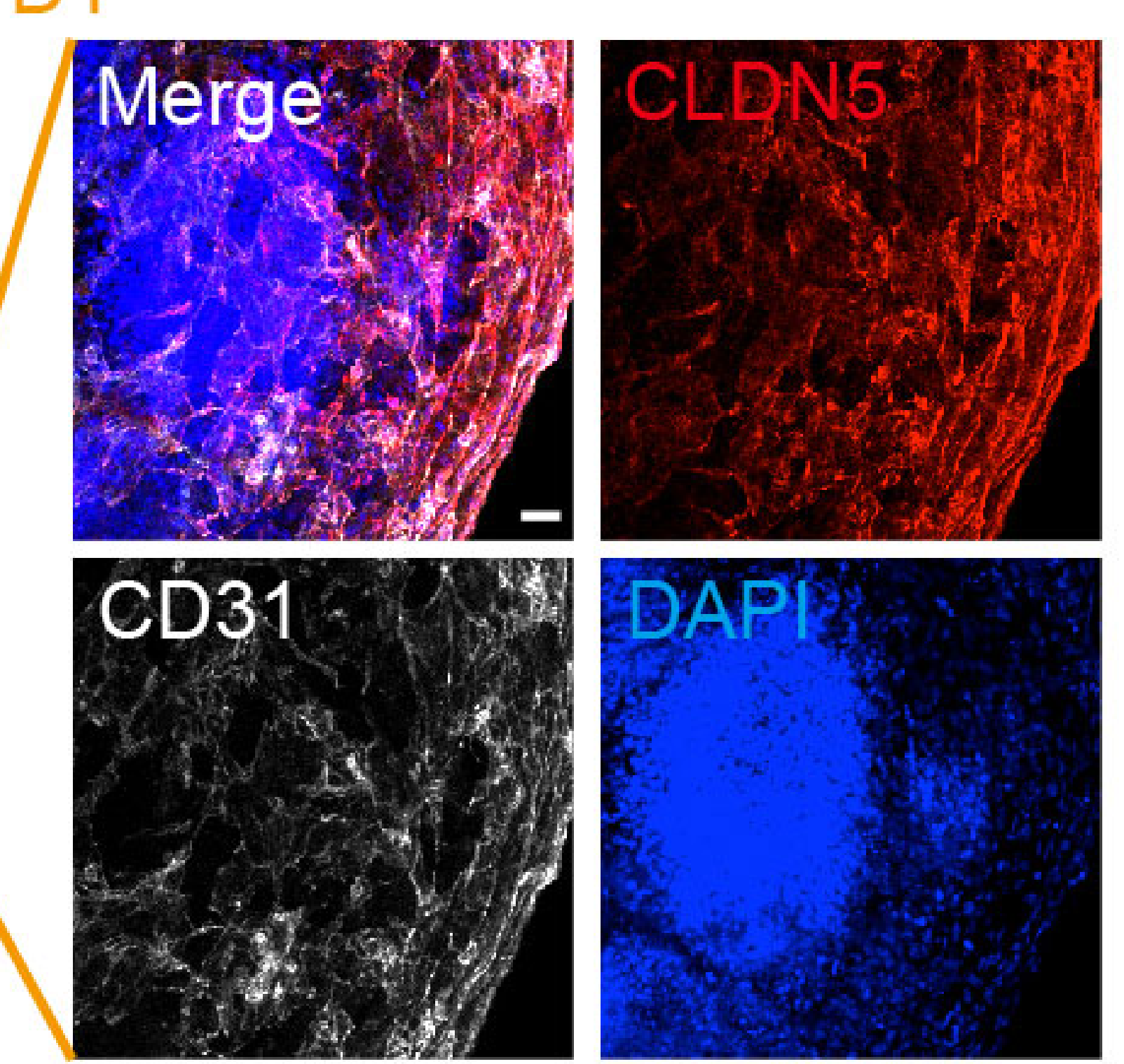

C

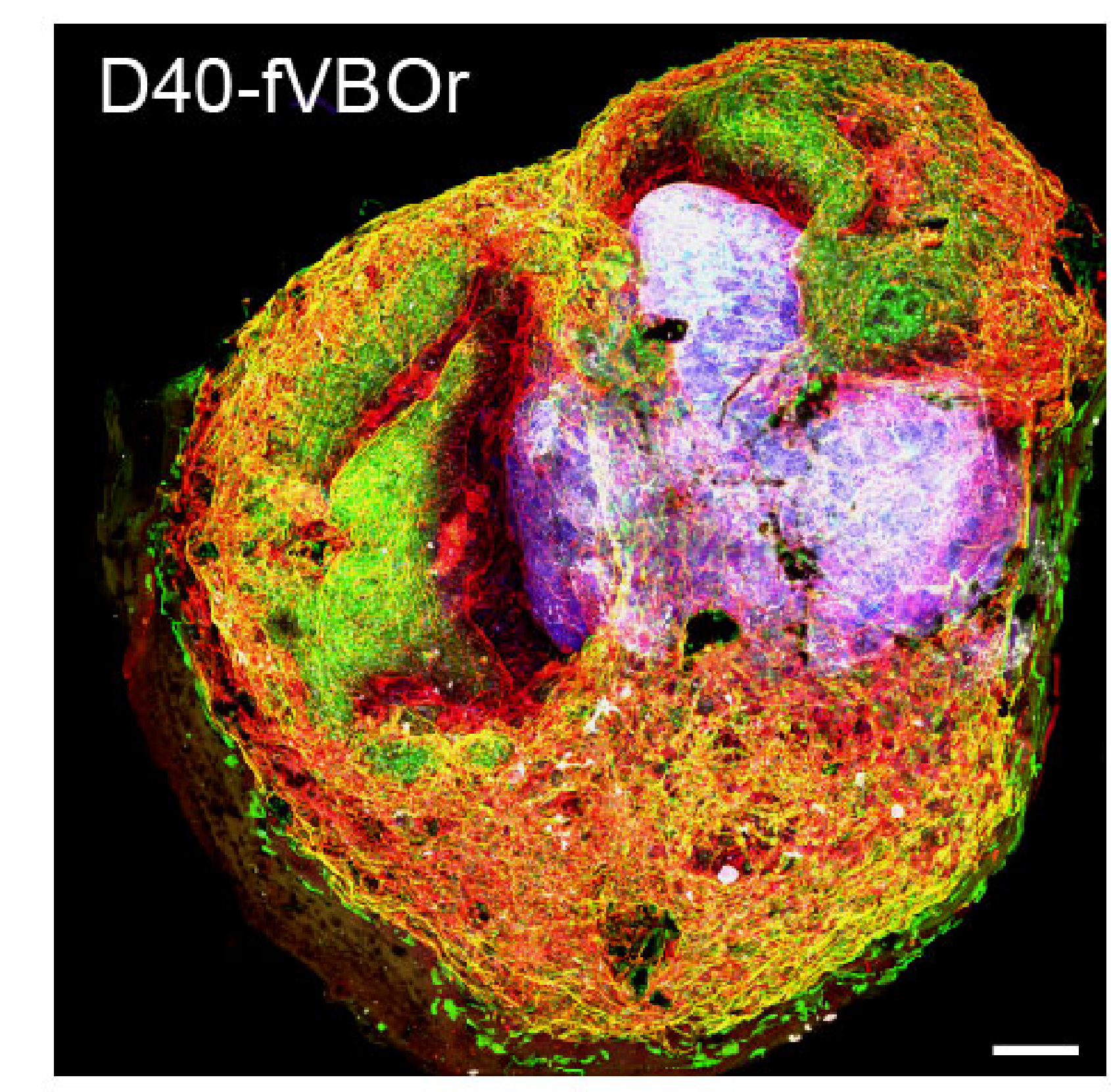

\section{DCX}
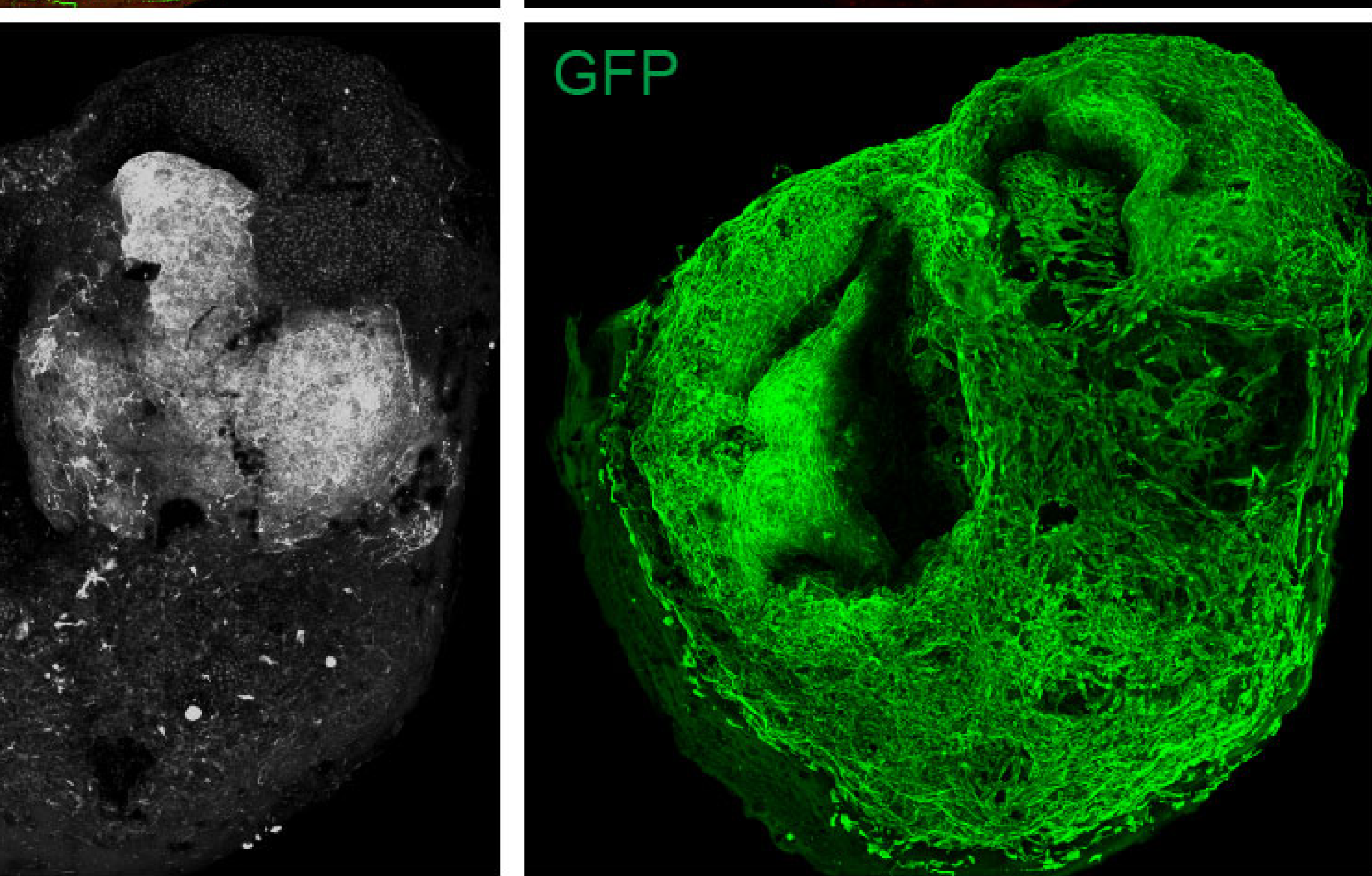

E

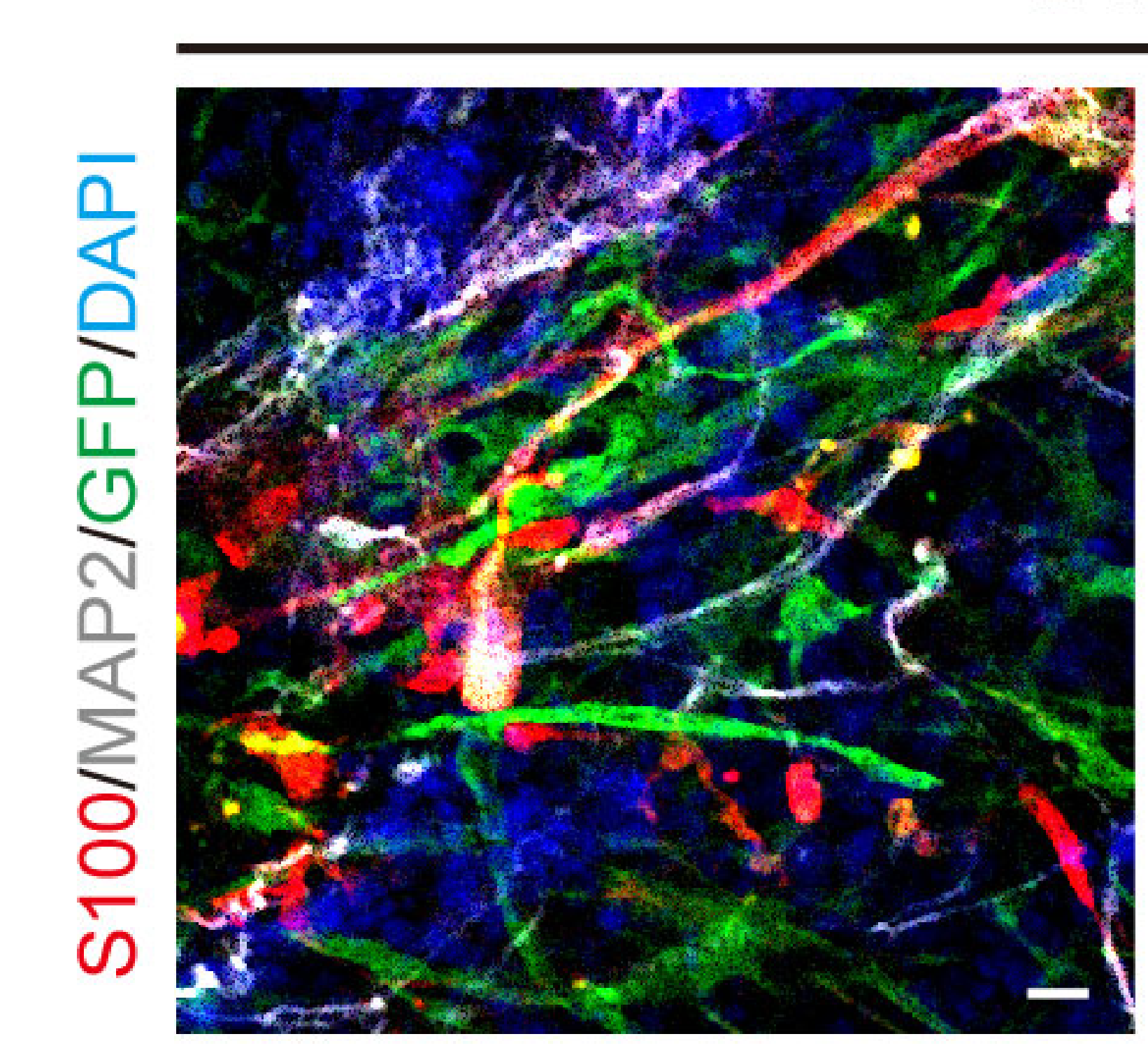

D42-fVBOr

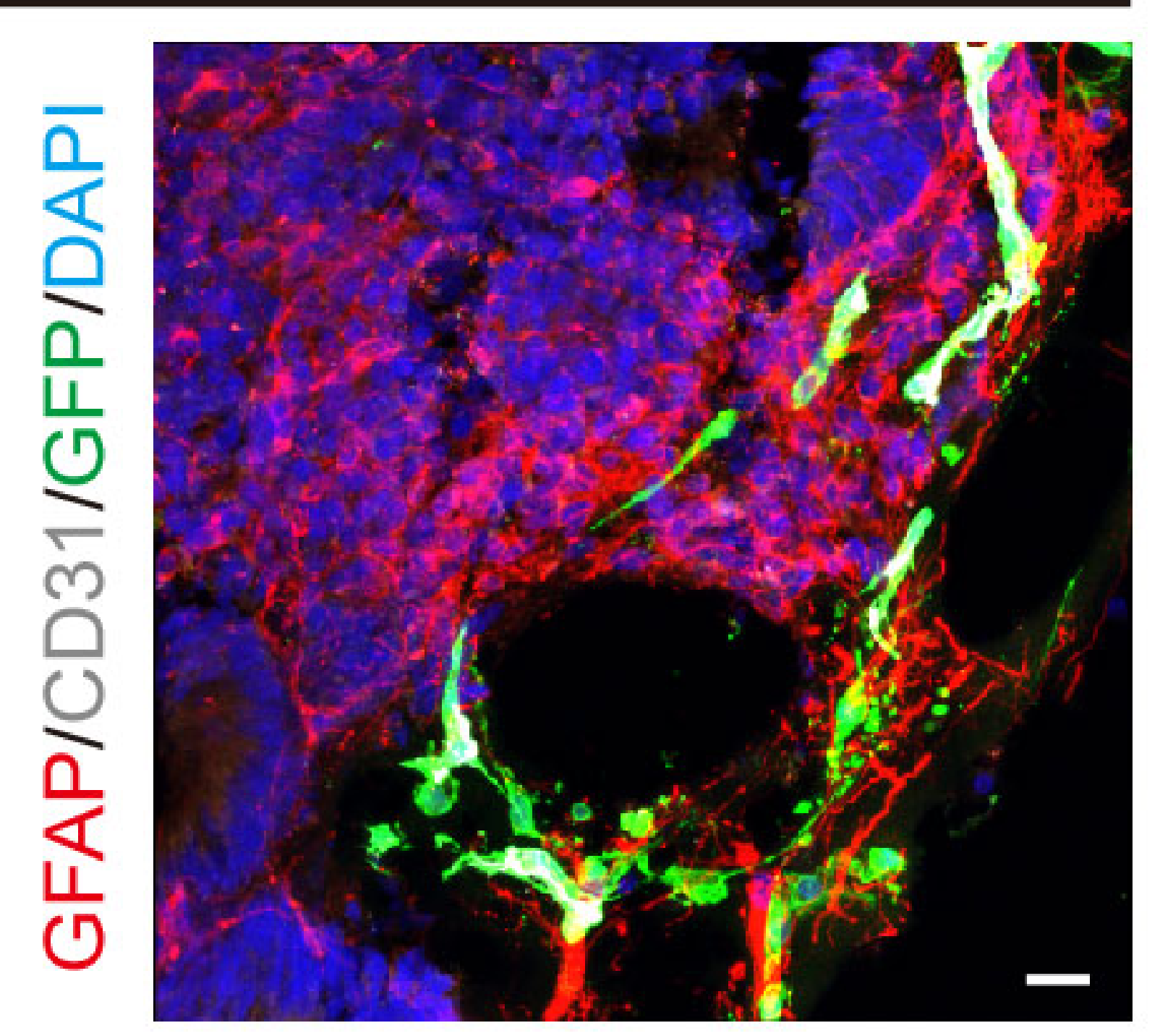

G
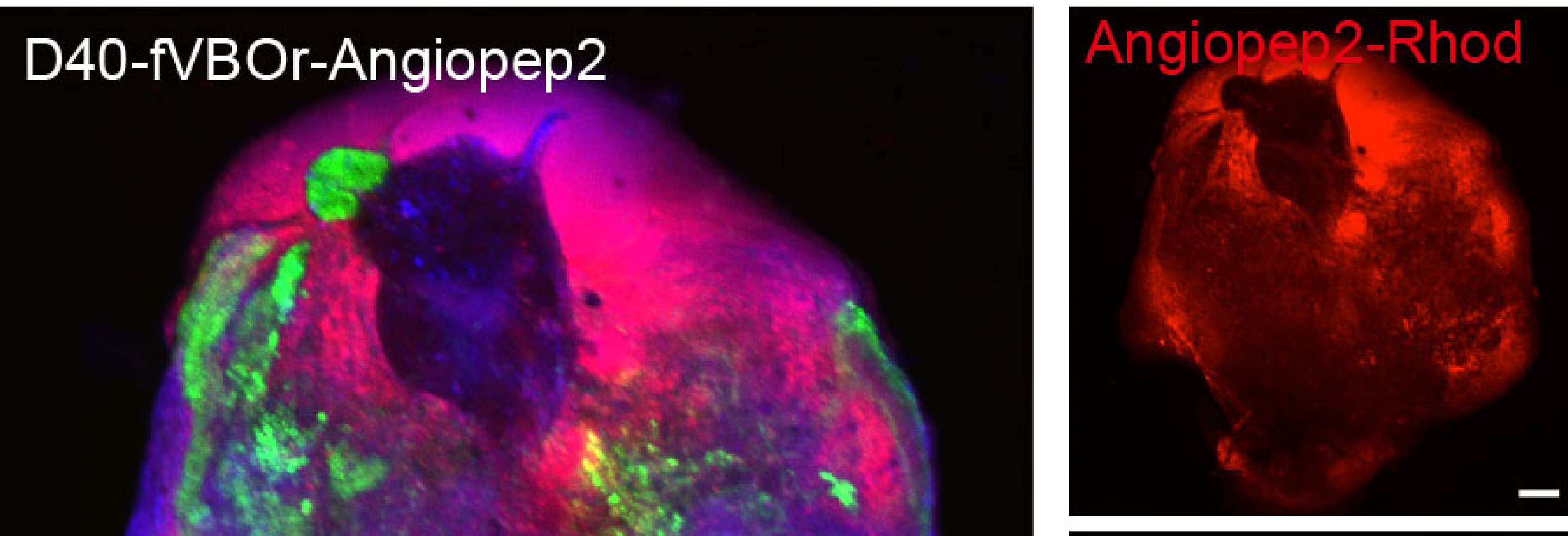

D40-fVBOr-Scramble

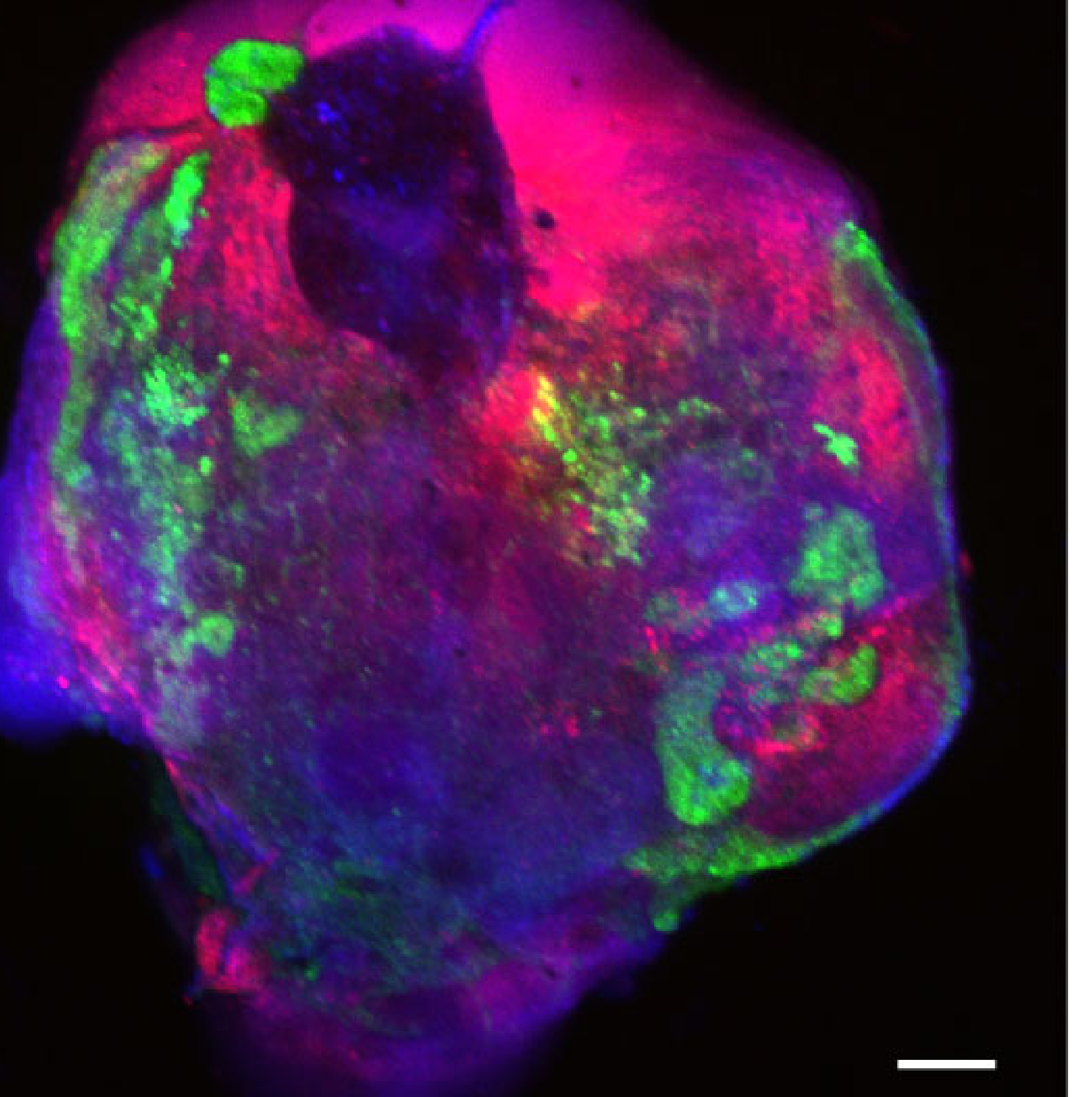



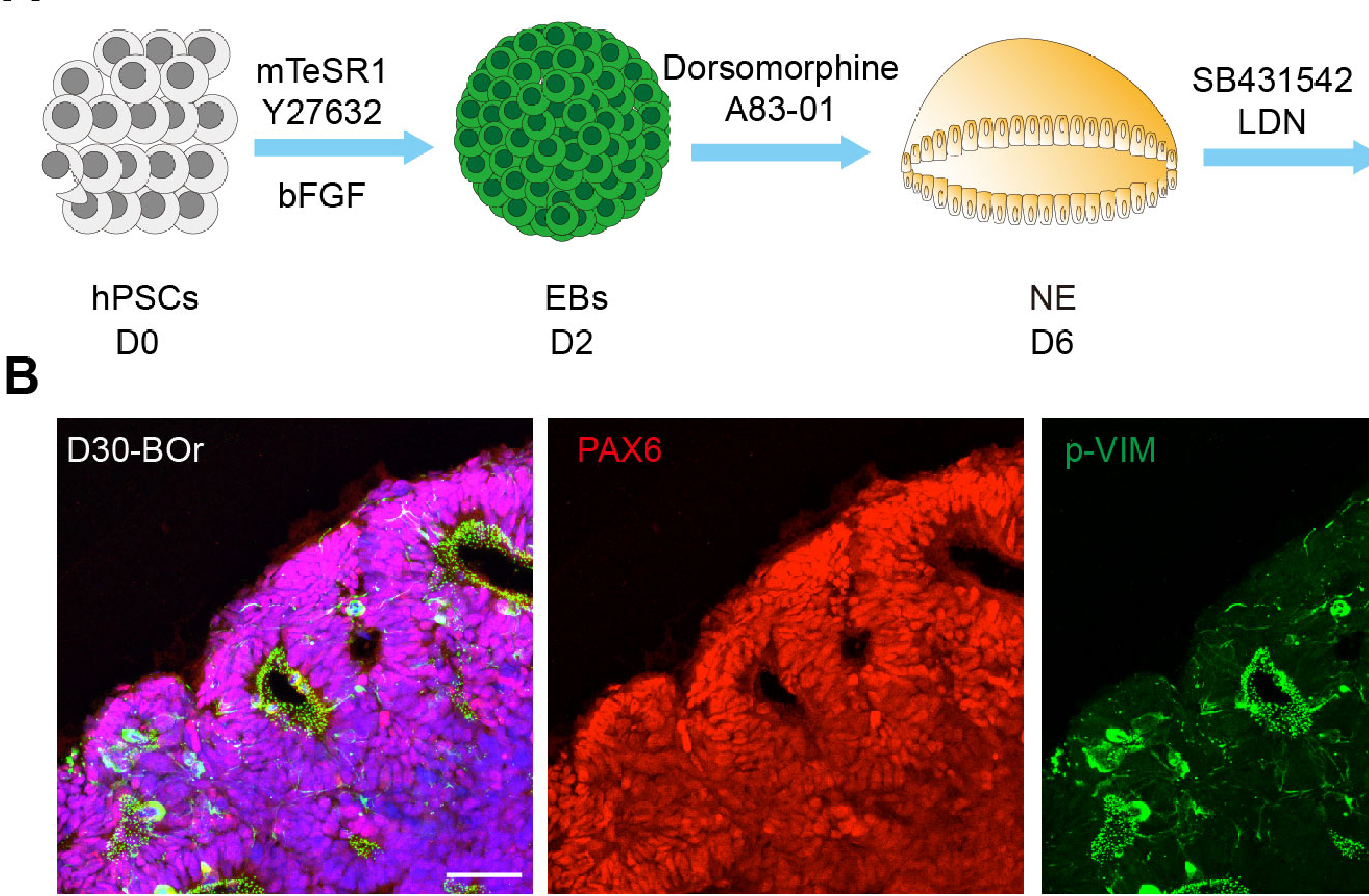

N2/B27

Maturation

C

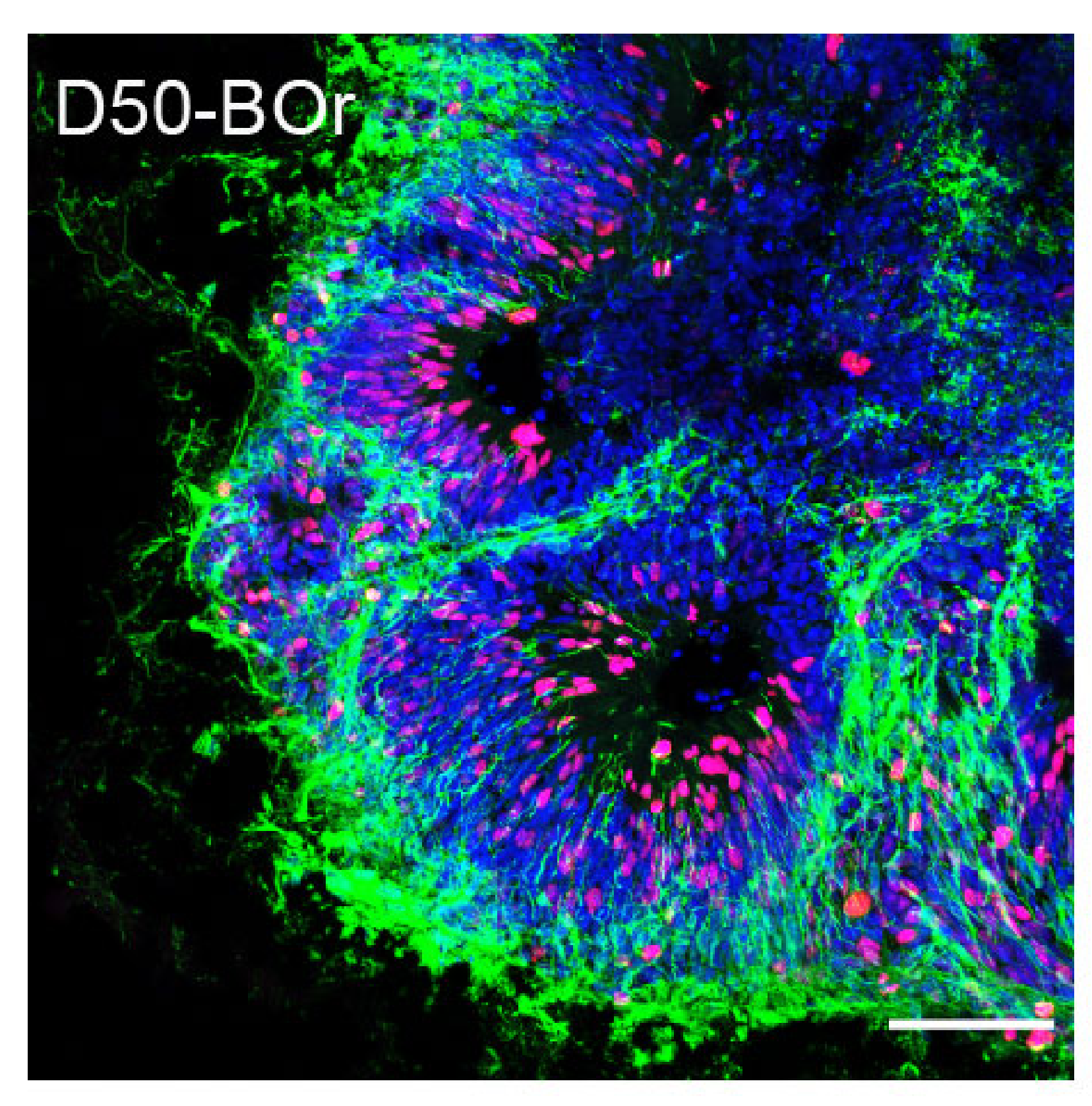

EBs

D2

NE

BOr

D6

D12
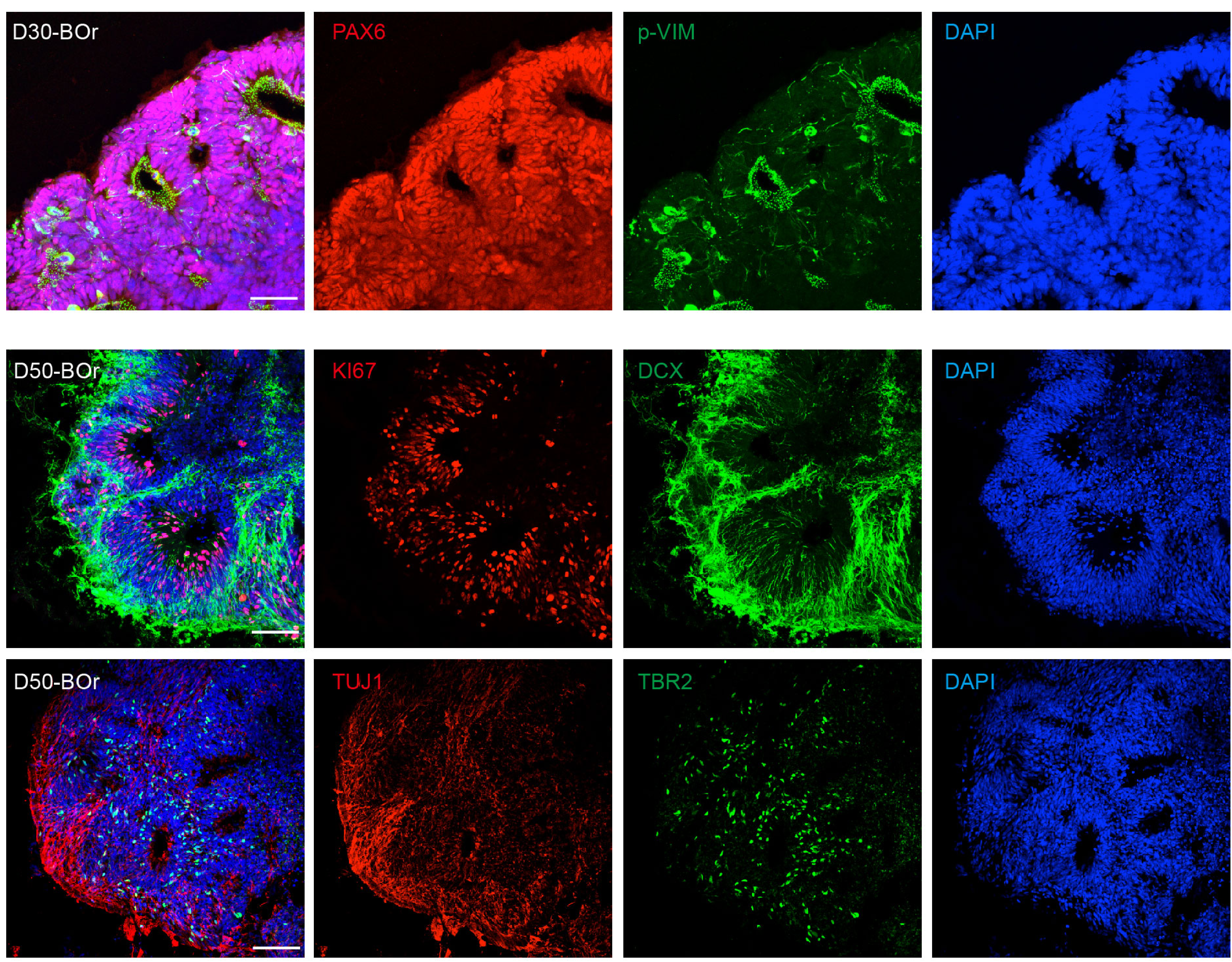

DAPI

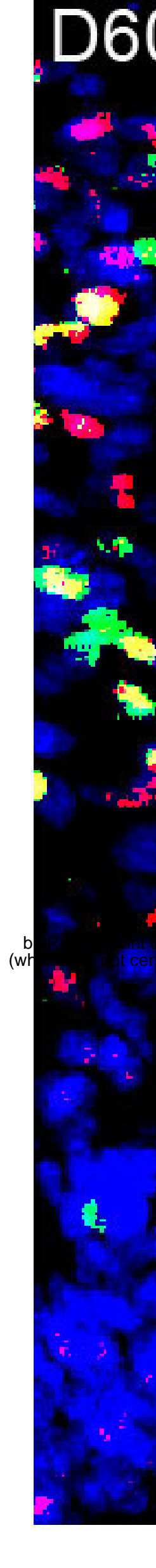

D60-BOr

D25-BOr

E
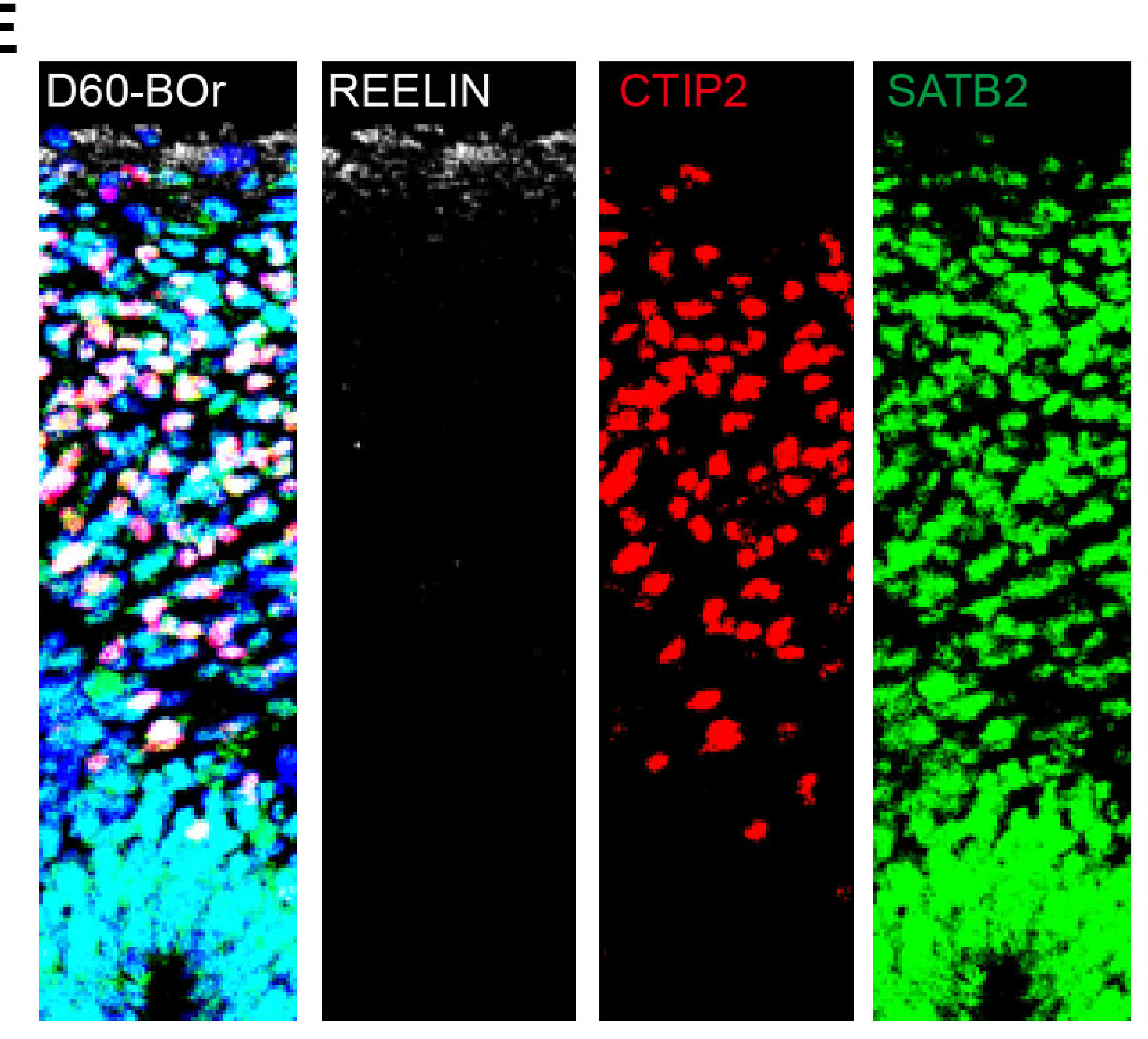

DAPI

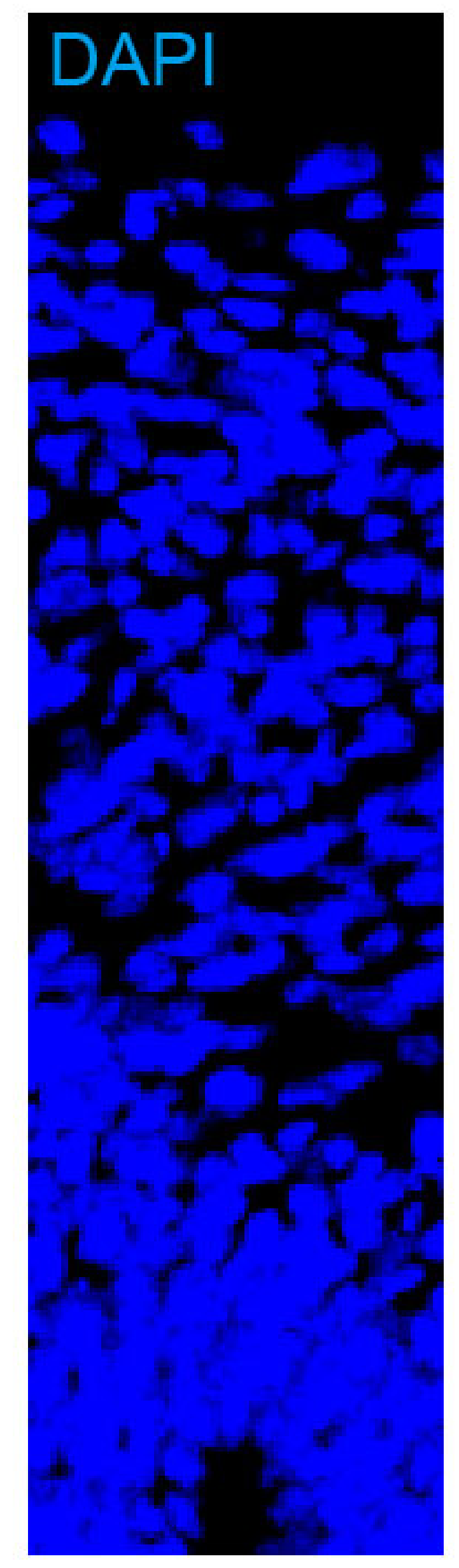




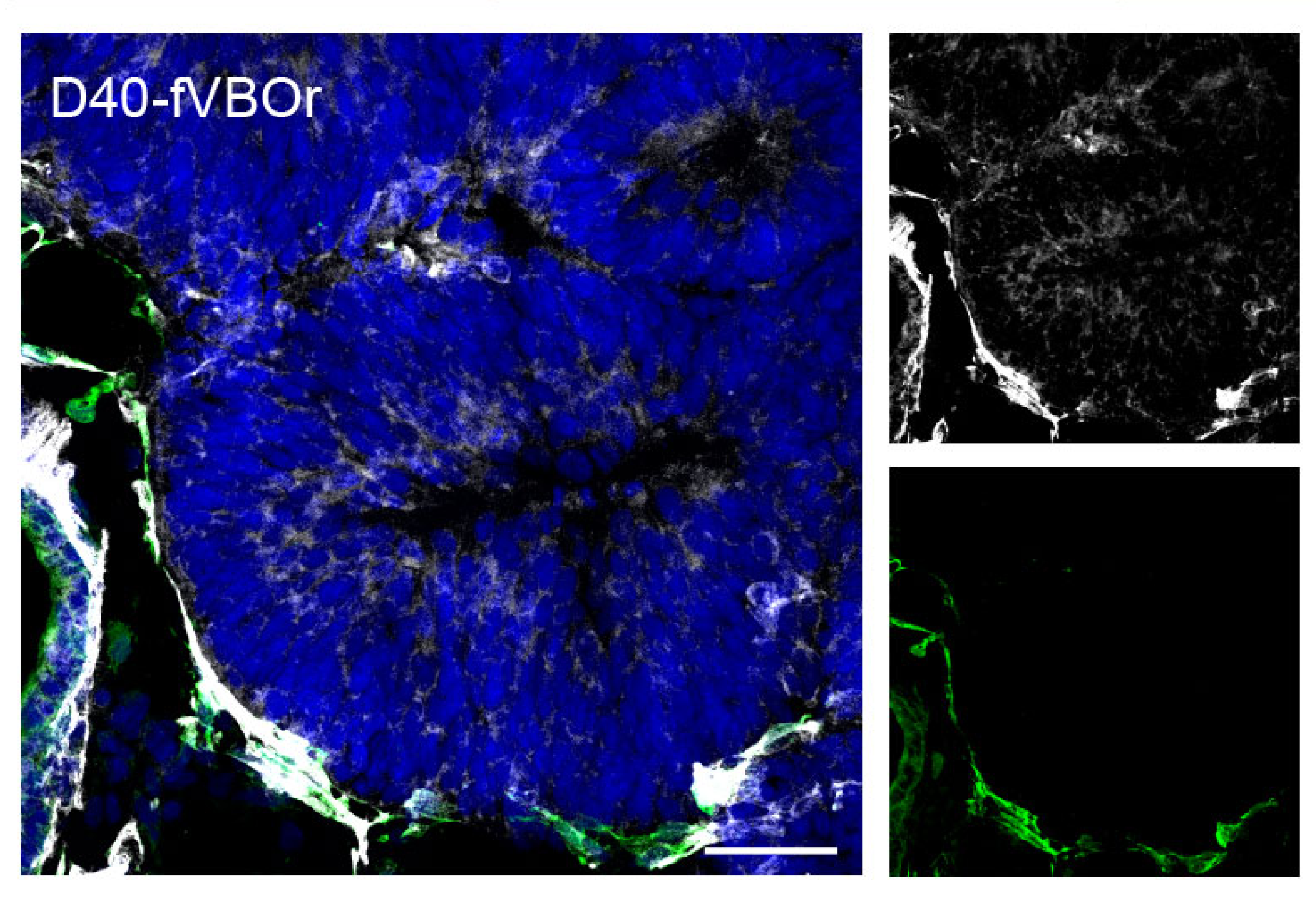

B

DAPI / GFP / ZO-1 / CD31

\section{D40-BOr}

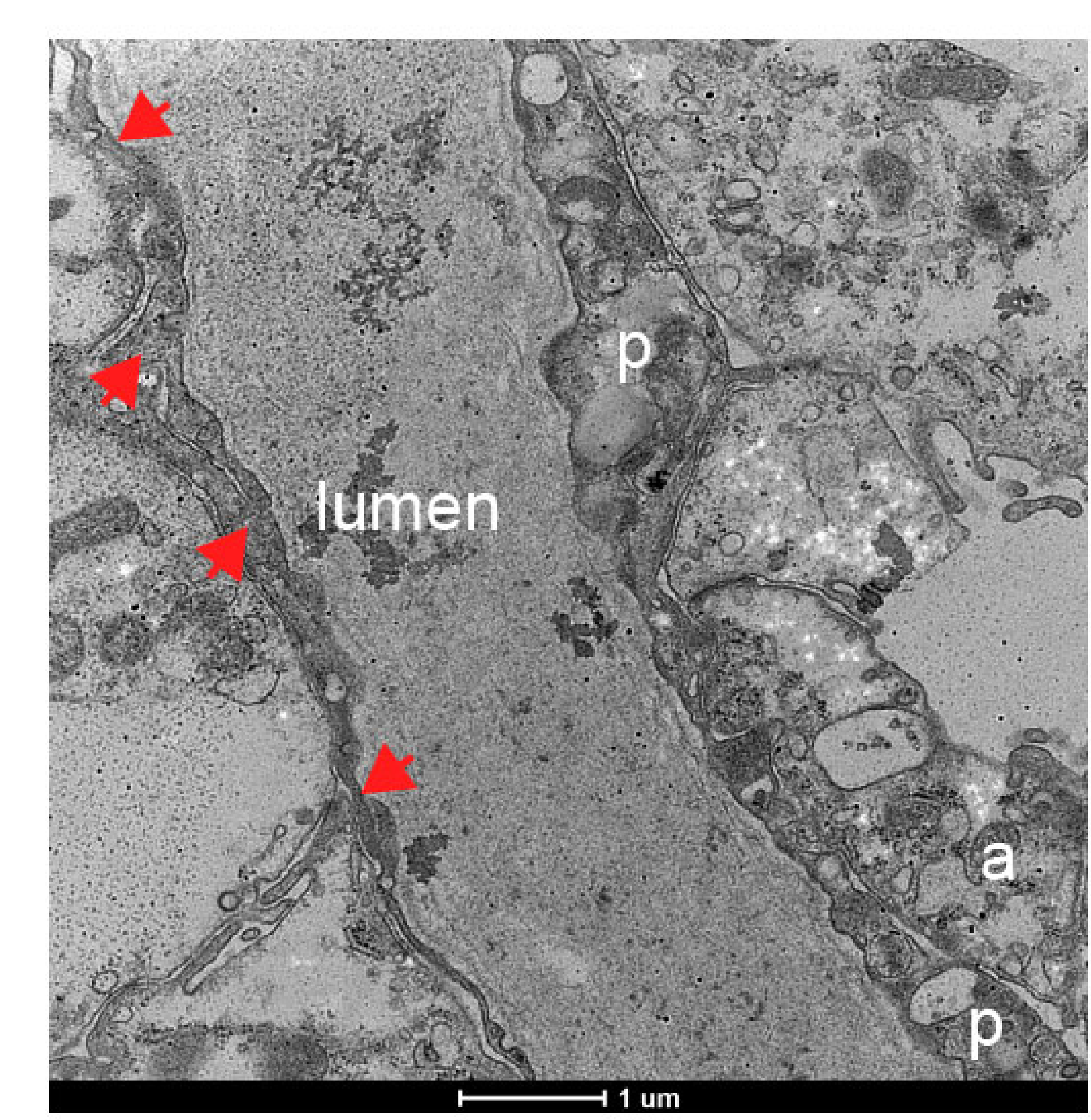

C

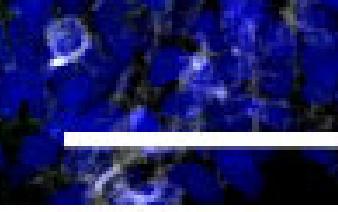

DAPI / GFP / p-GLYCOPROTEIN / CD31

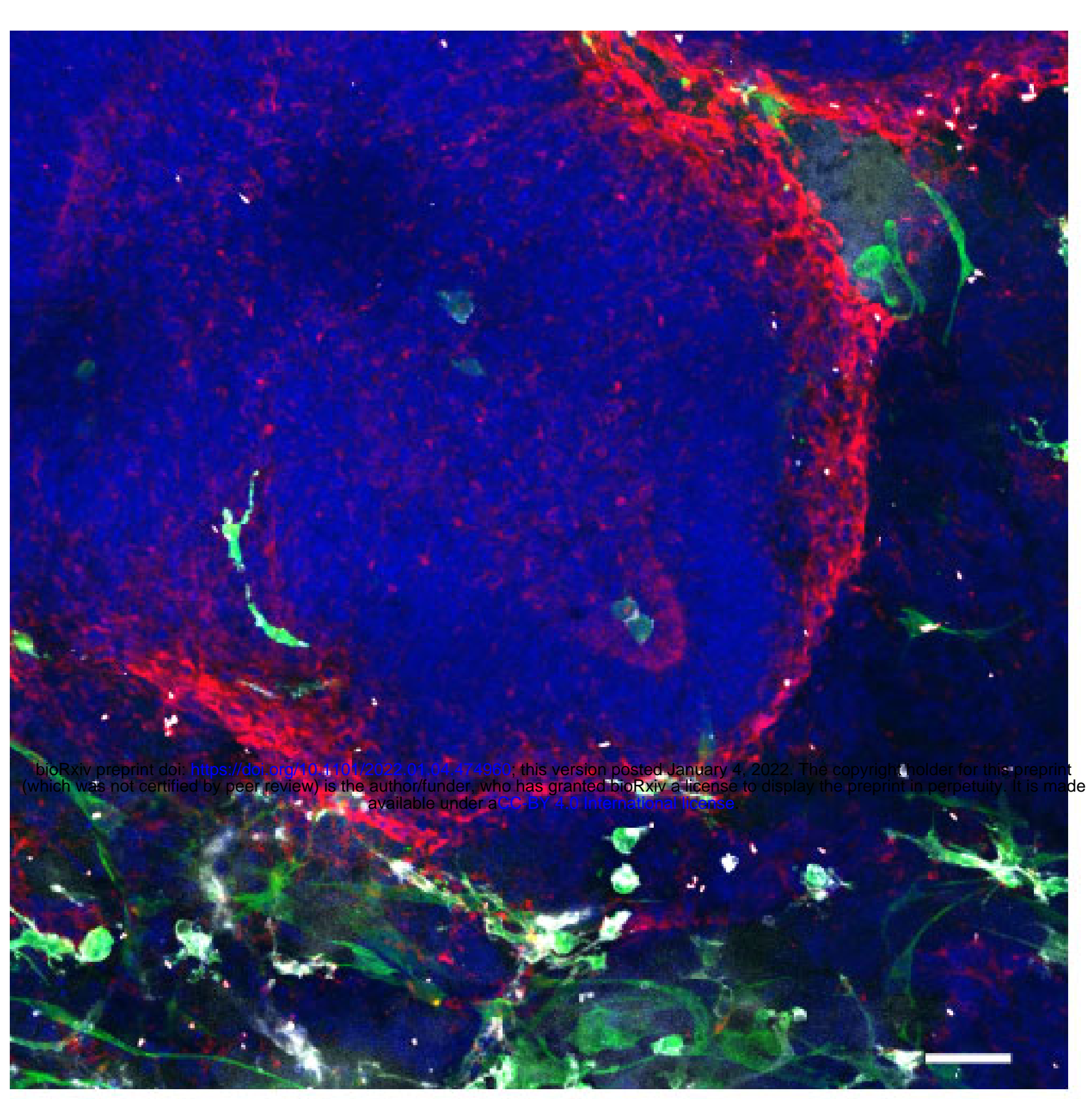

E

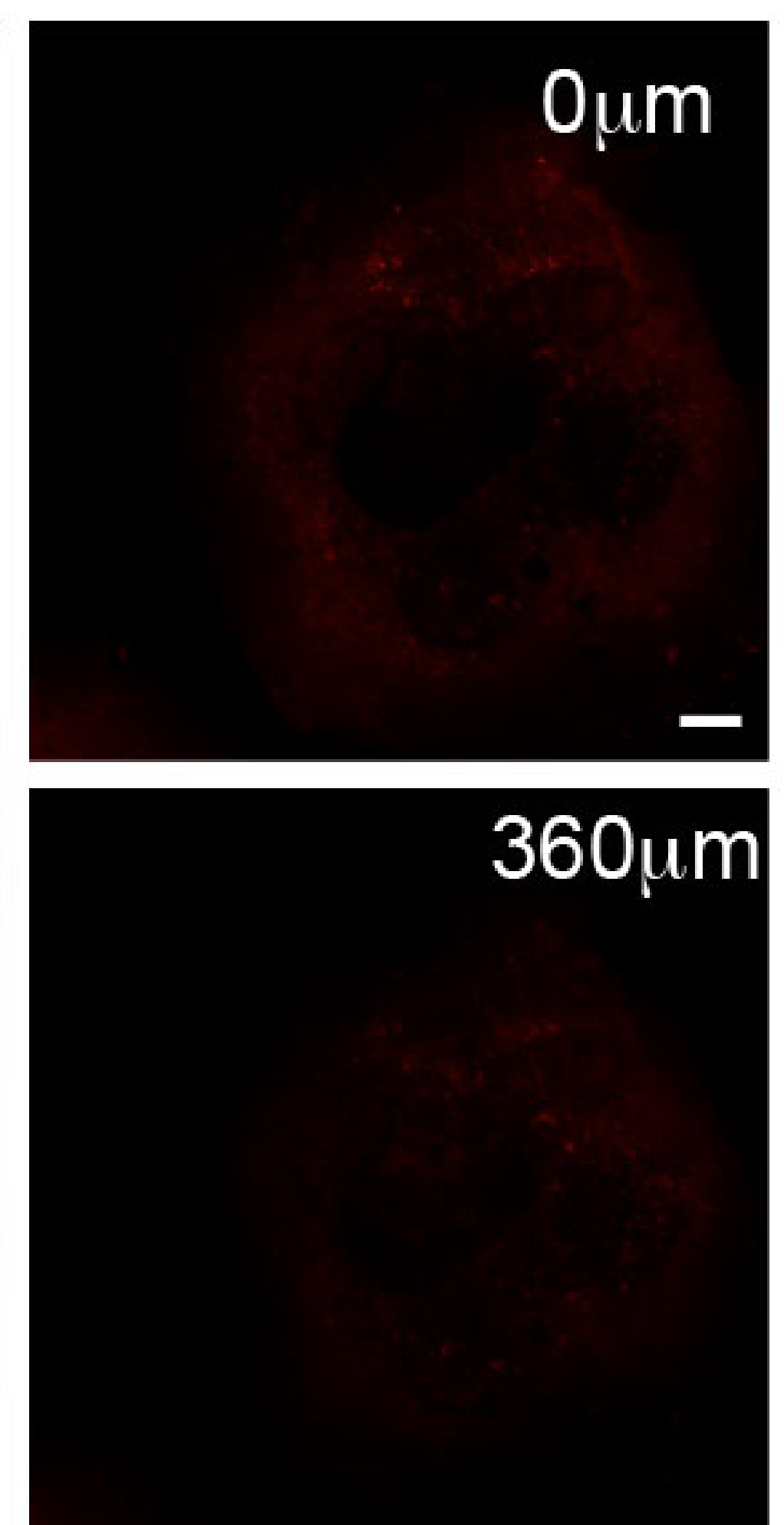

$480 \mu \mathrm{m}$

II.
F

\section{$600 \mu \mathrm{m}$}
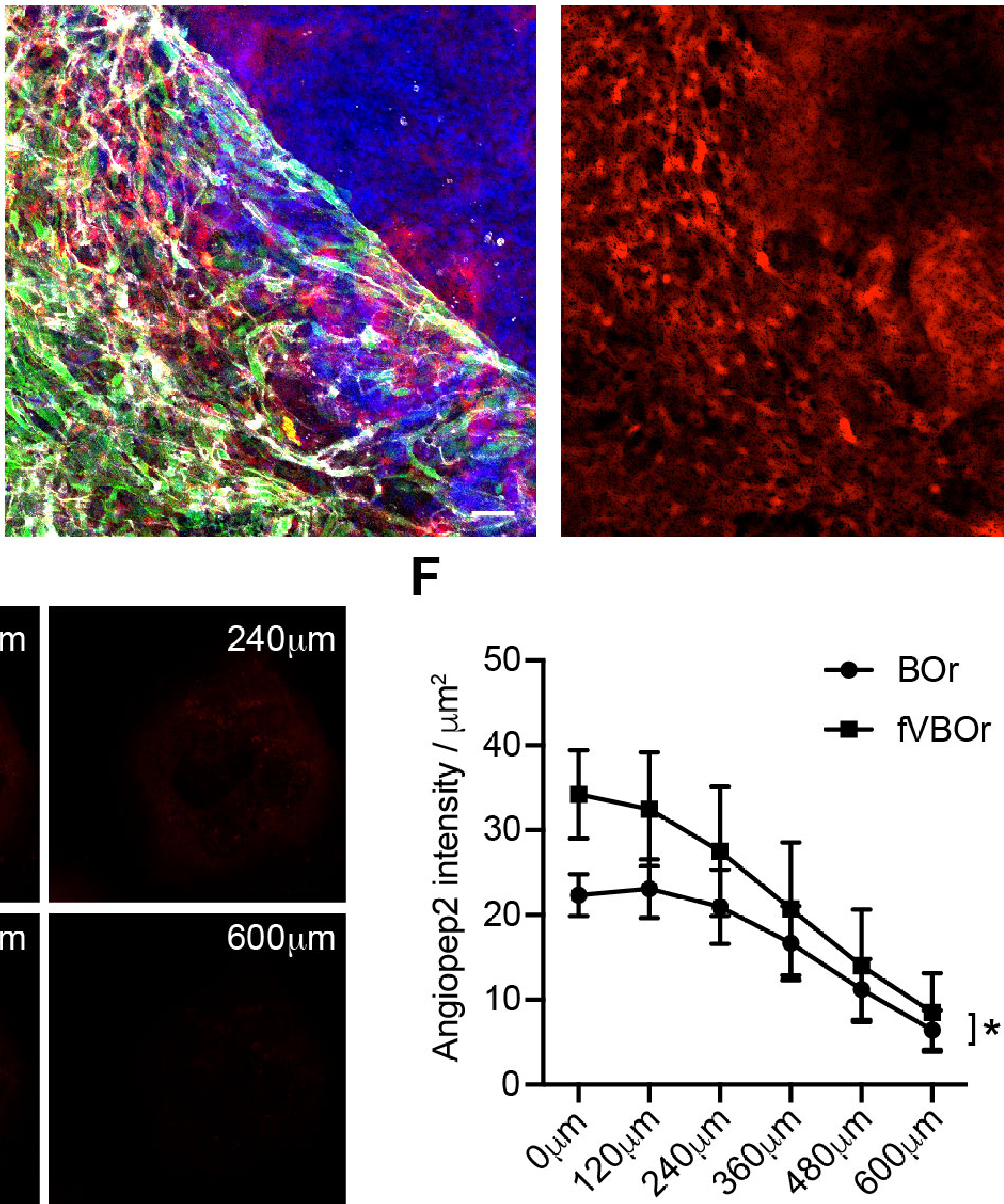
$\mathbf{A}$

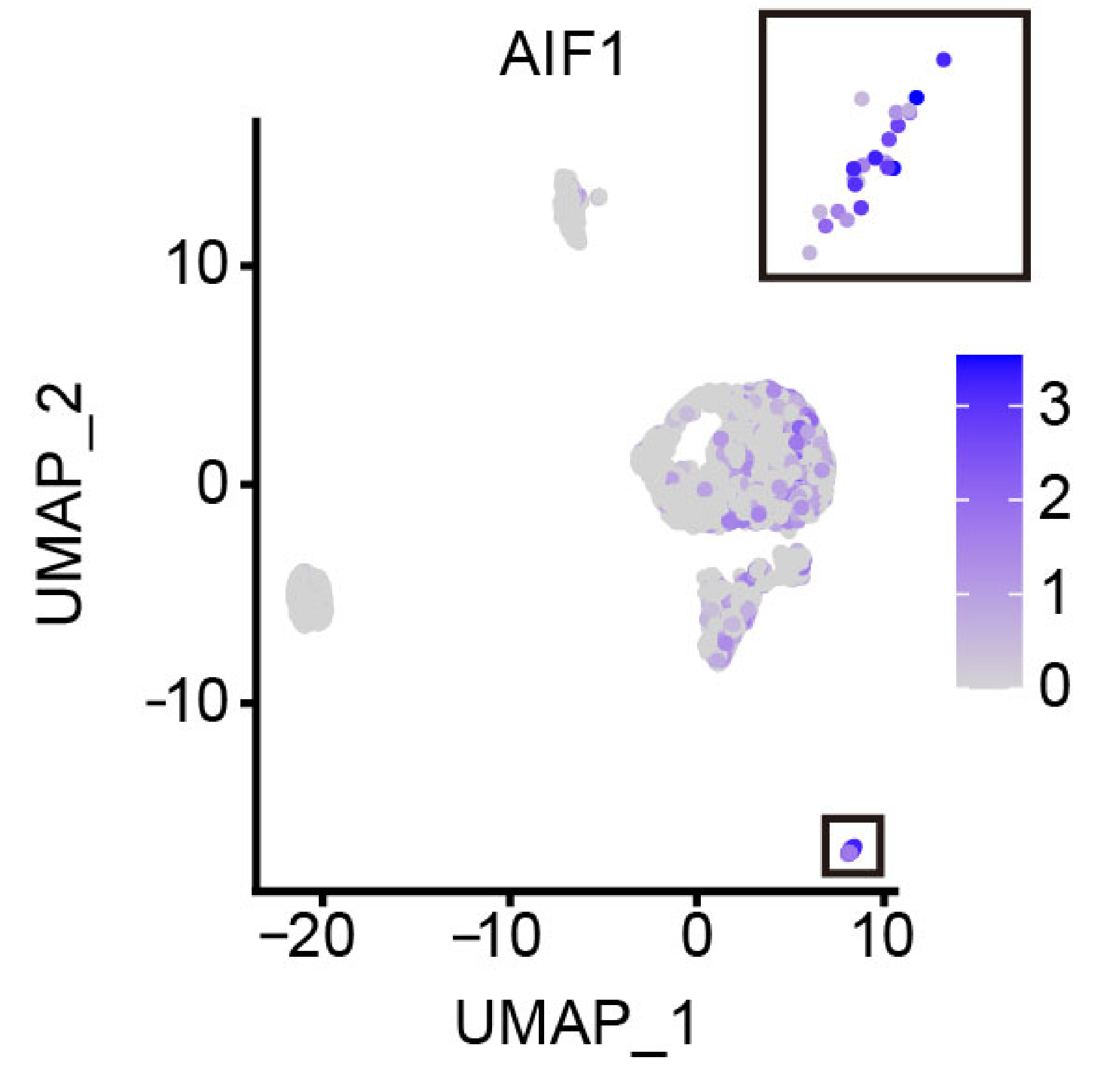

B
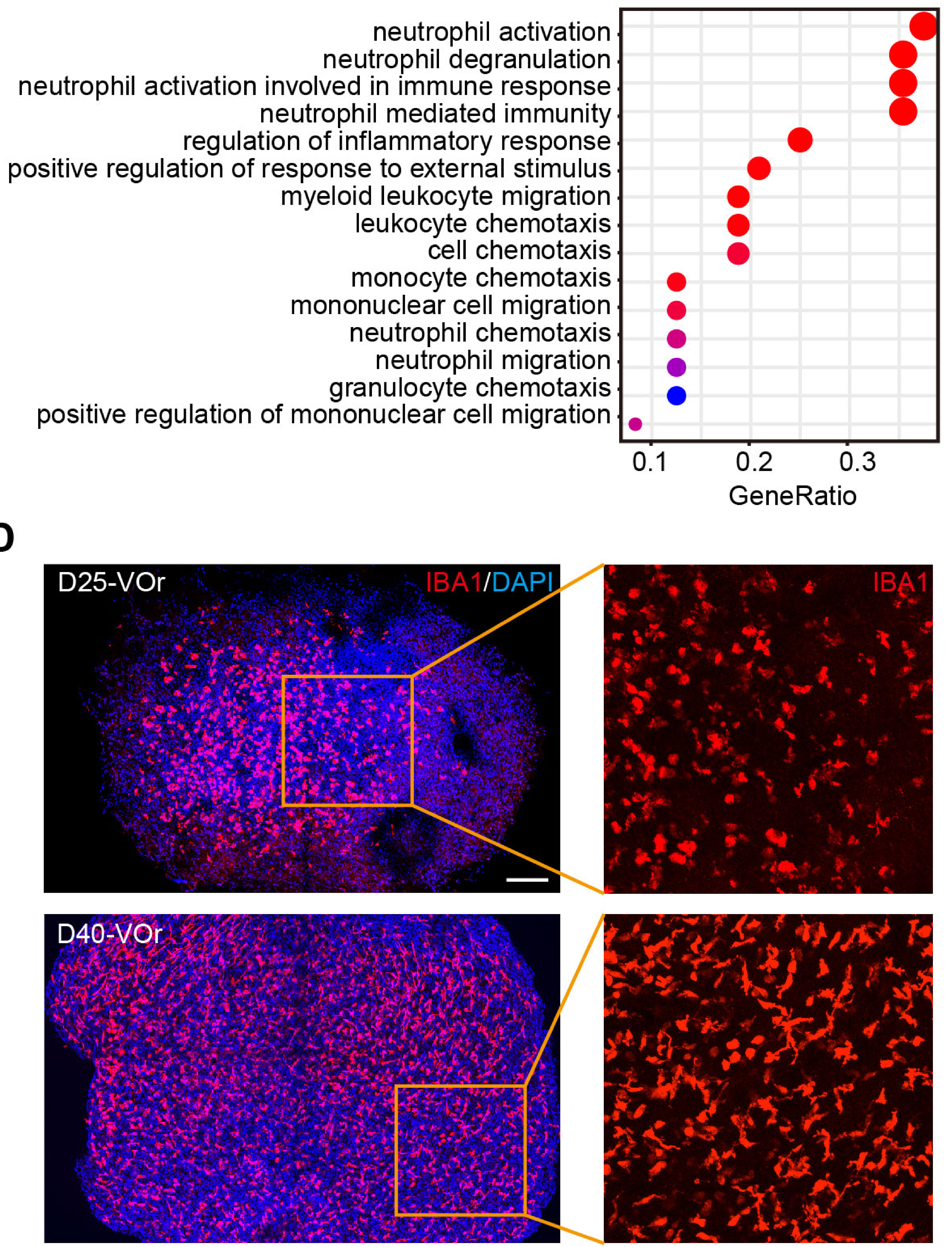

$\mathbf{F}$
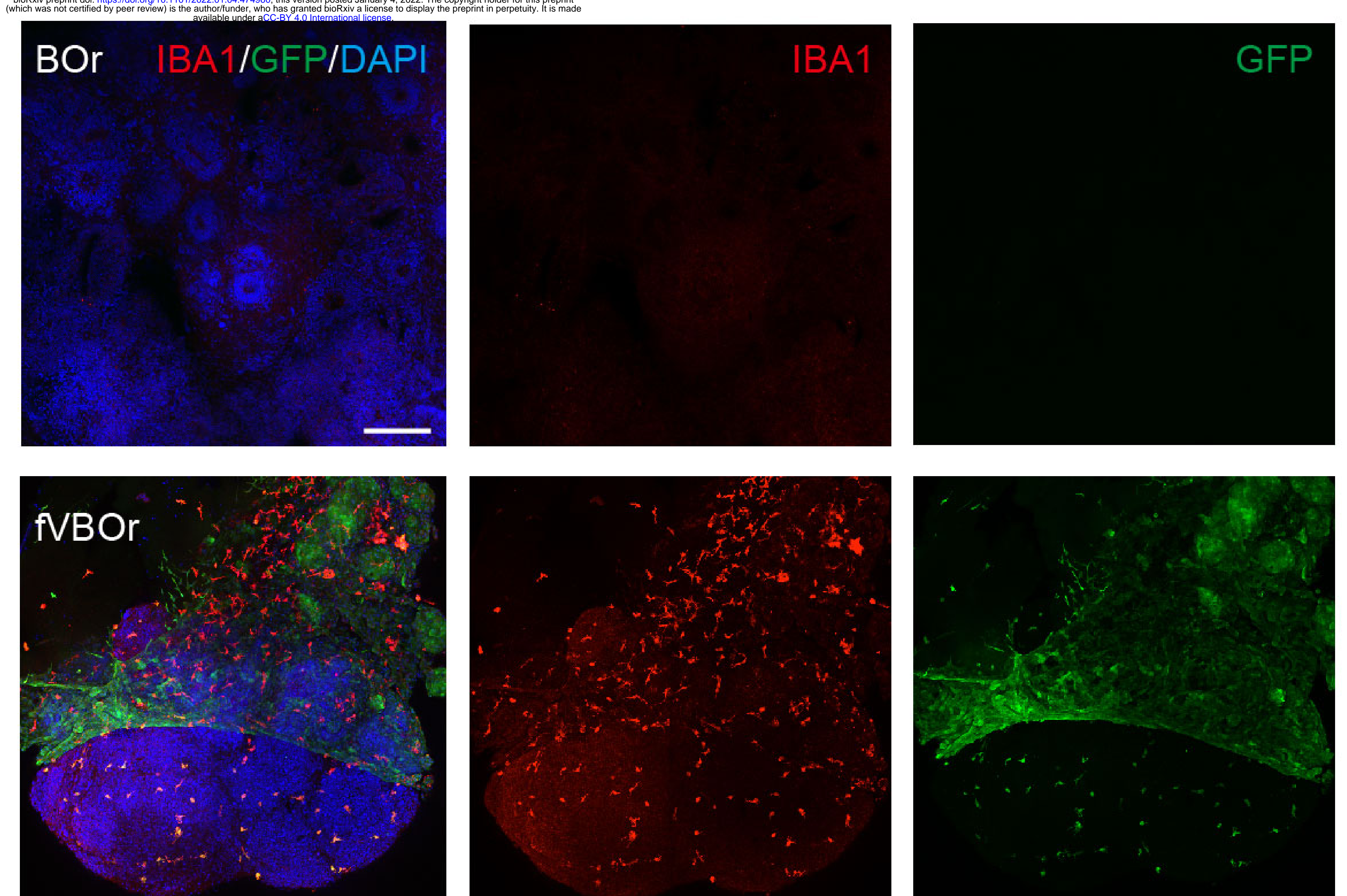

Count

- 4

8

12

16

p.adjust

$-2 e-05$

$-4 e-05$

-6e-05

$\mathbf{E}$

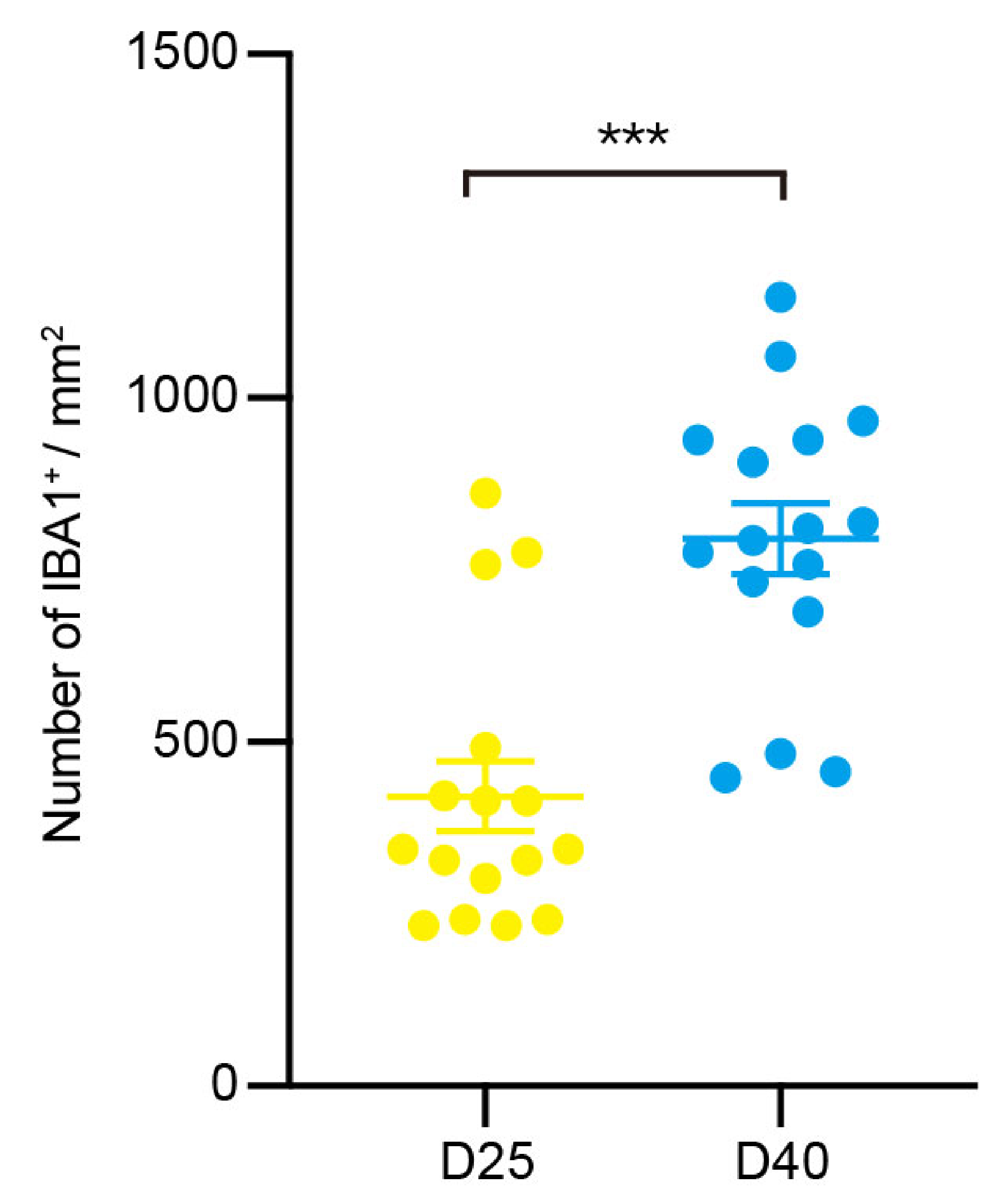

G

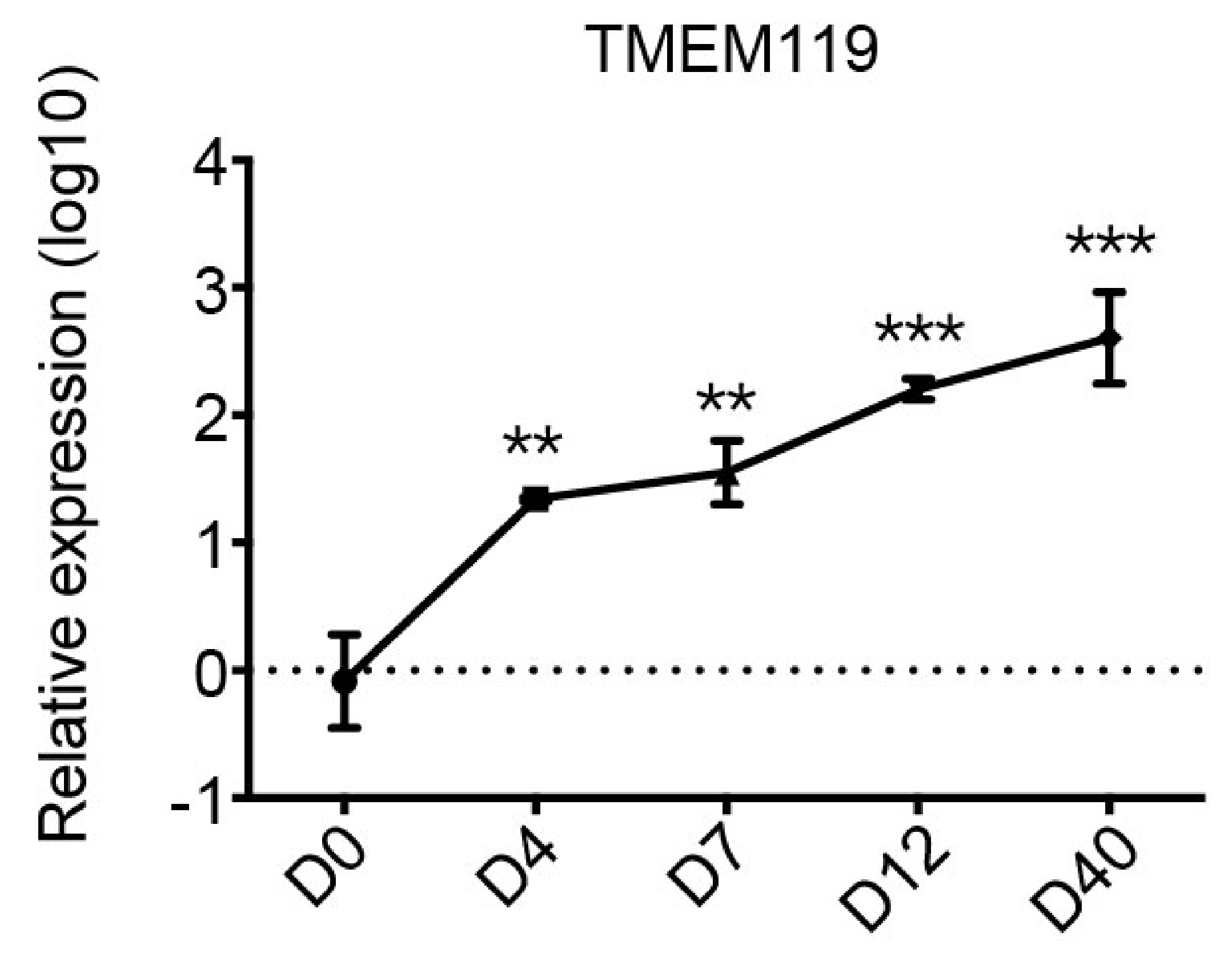

\section{E}

$\square$ DMSO $\square$ DMSO+LPS $\square$ PLX5622+LPS

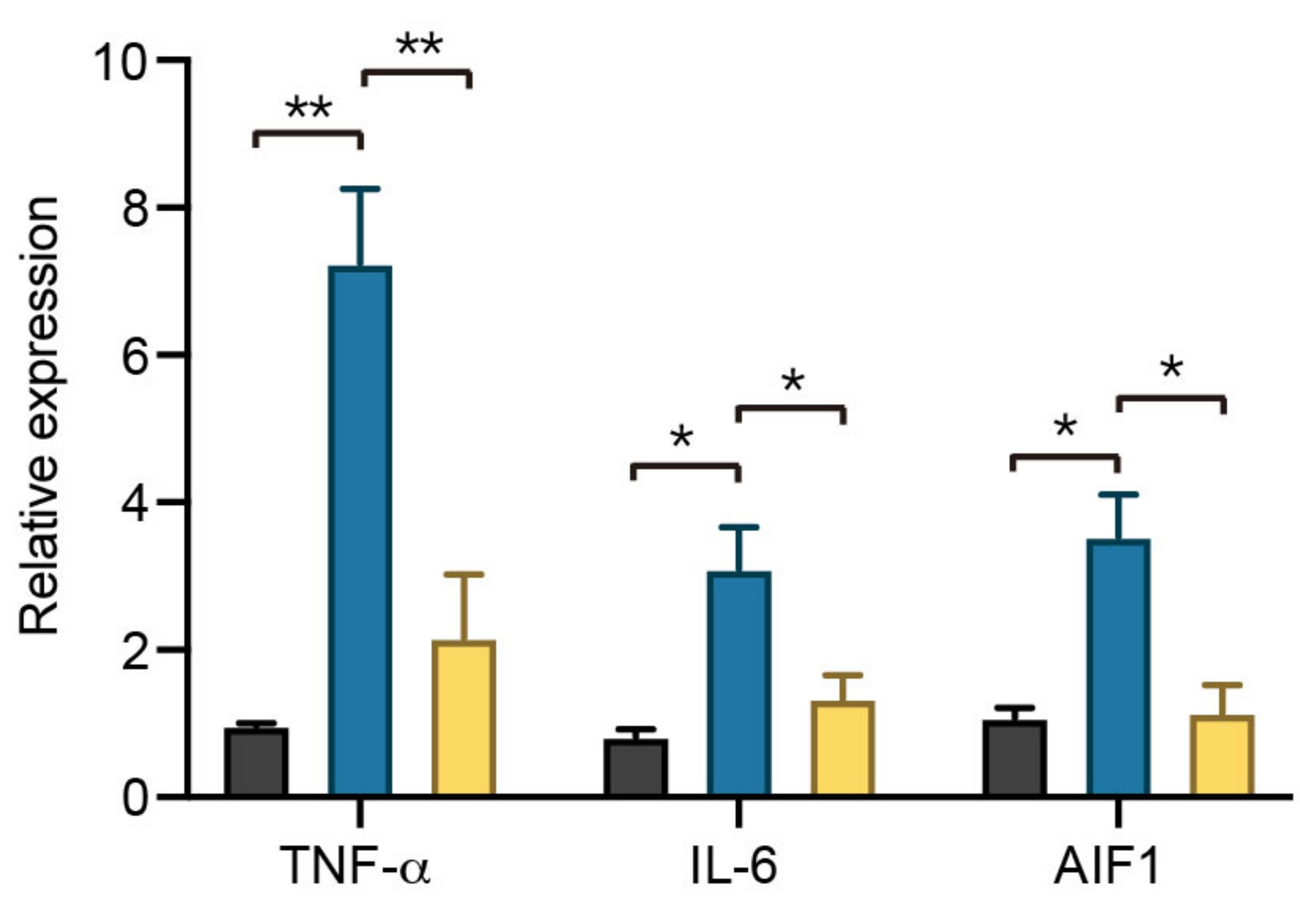



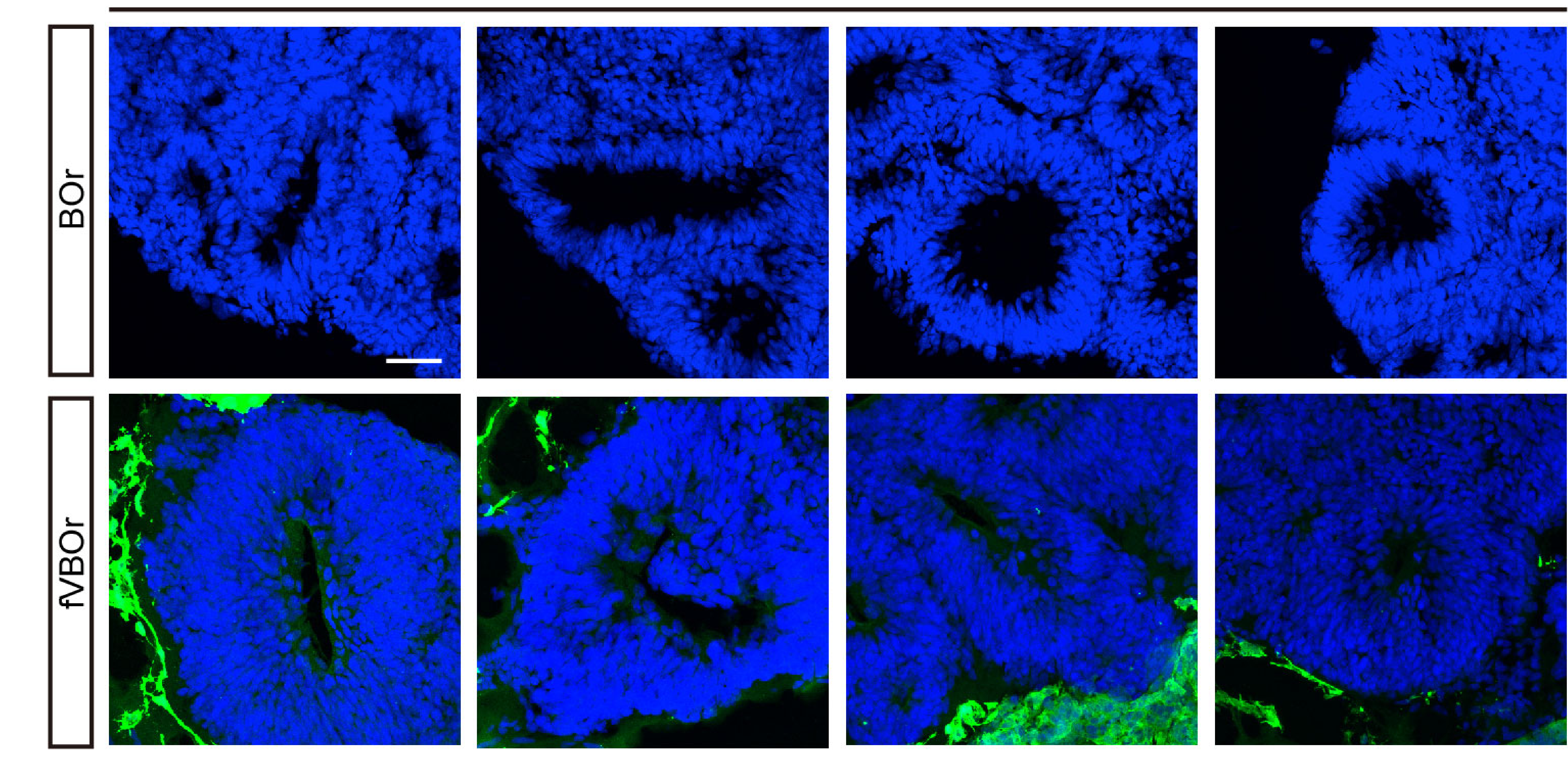

PAX6/p-VIM/GFP/DAPI
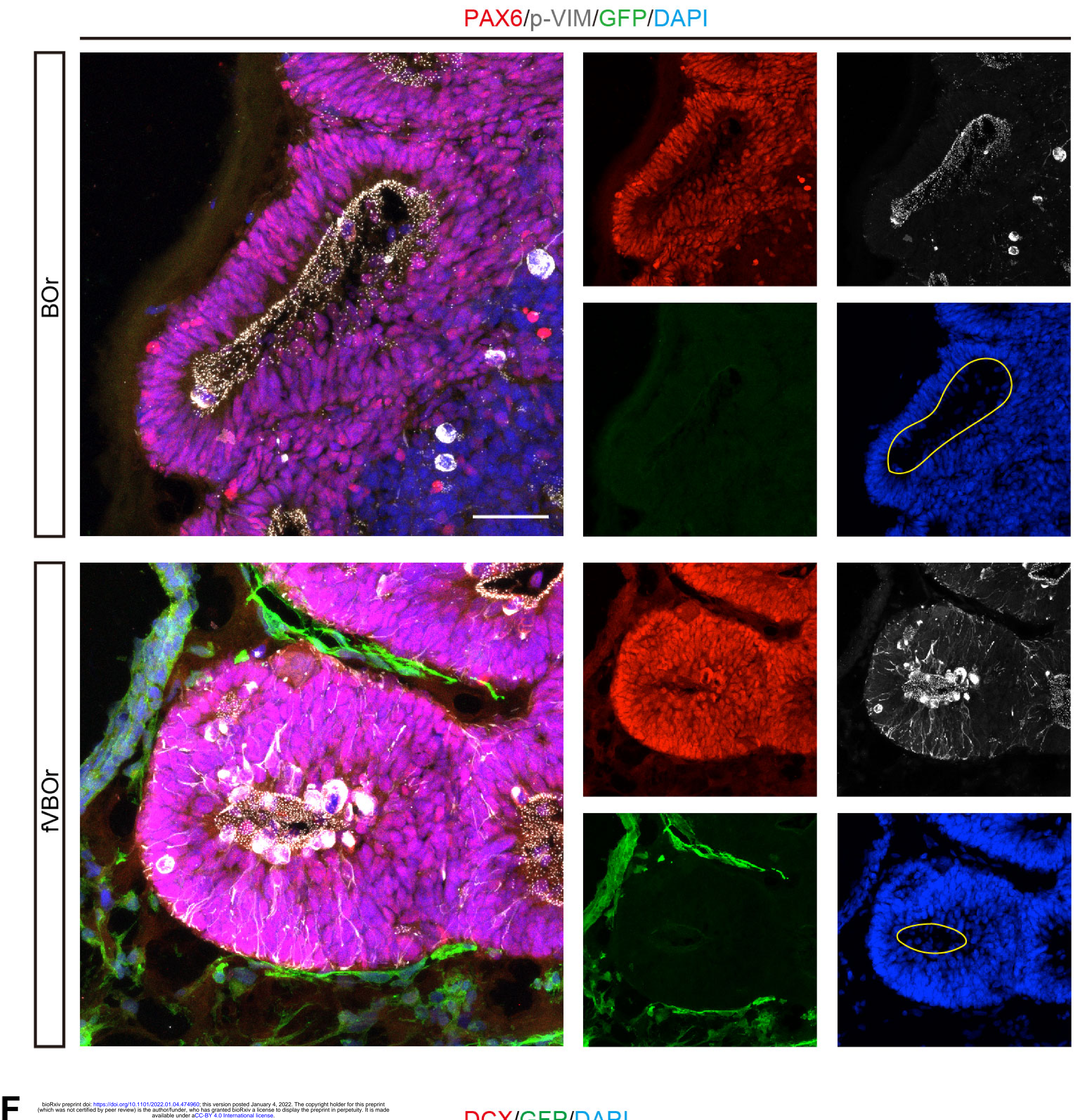

$\mathbf{F}$

DCX/GFP/DAPI
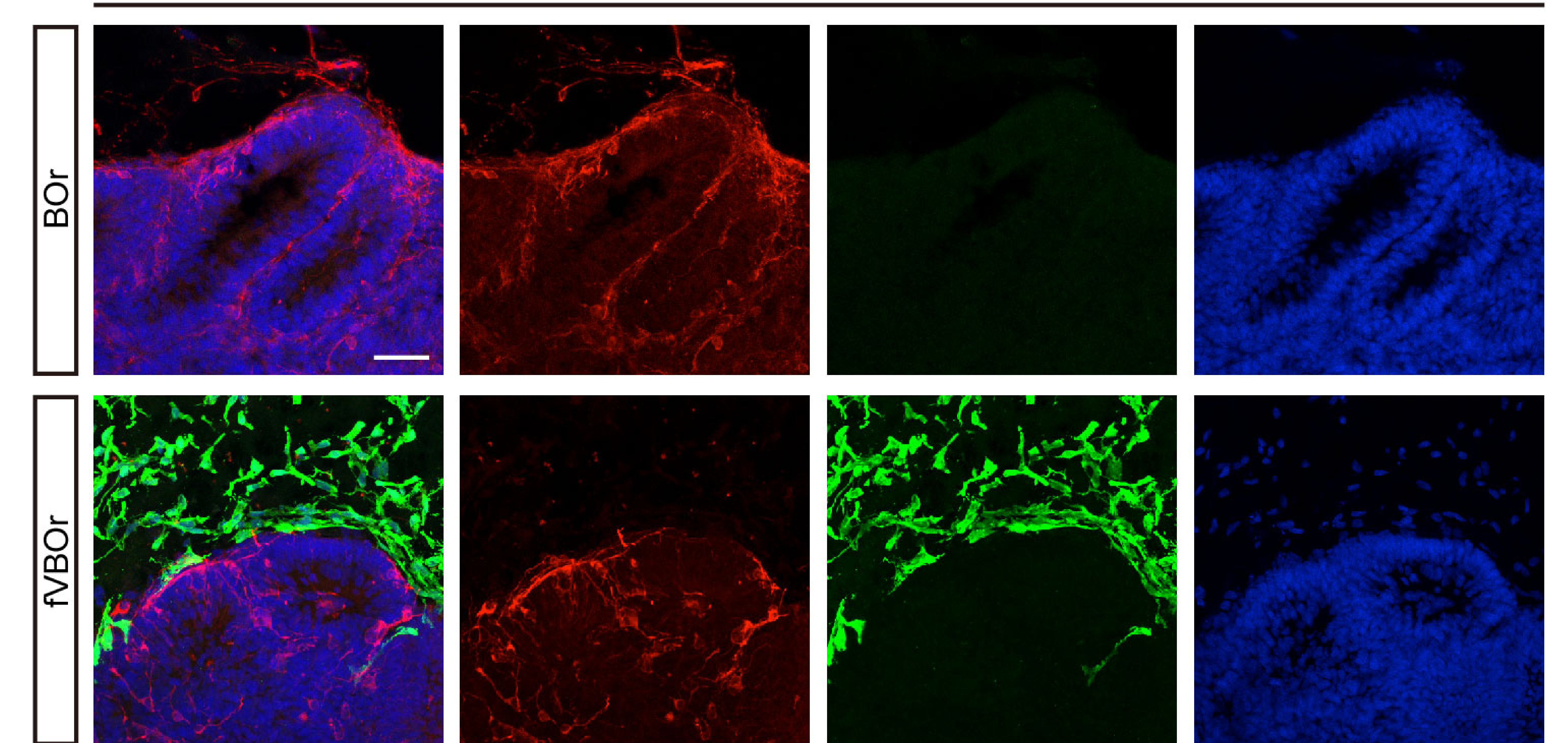

B

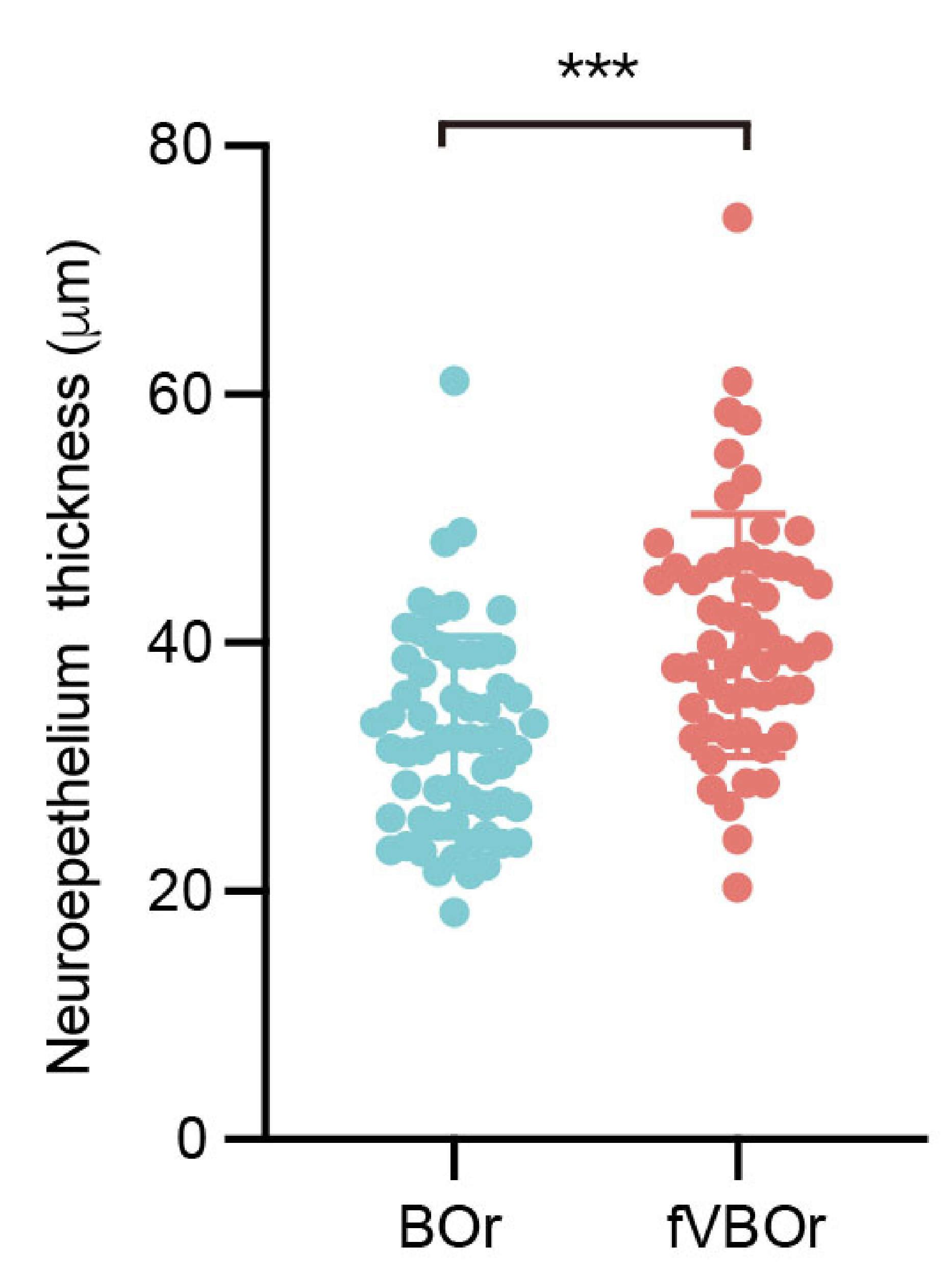

D
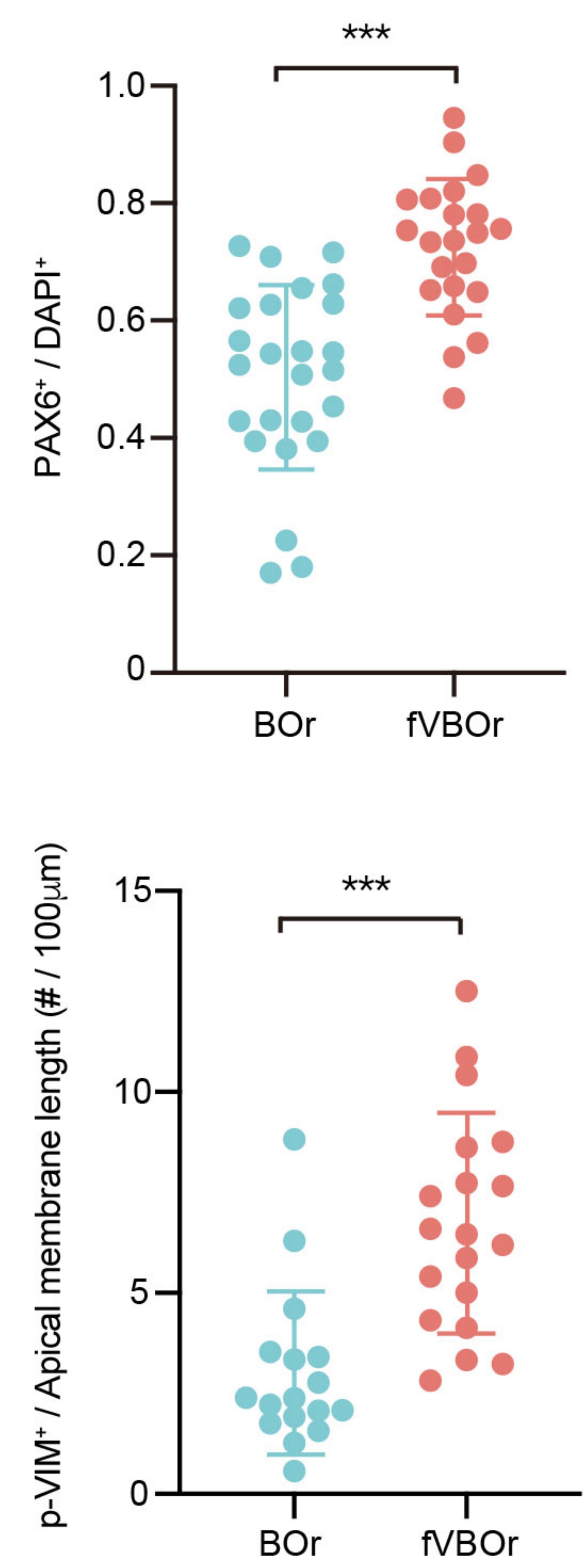

G

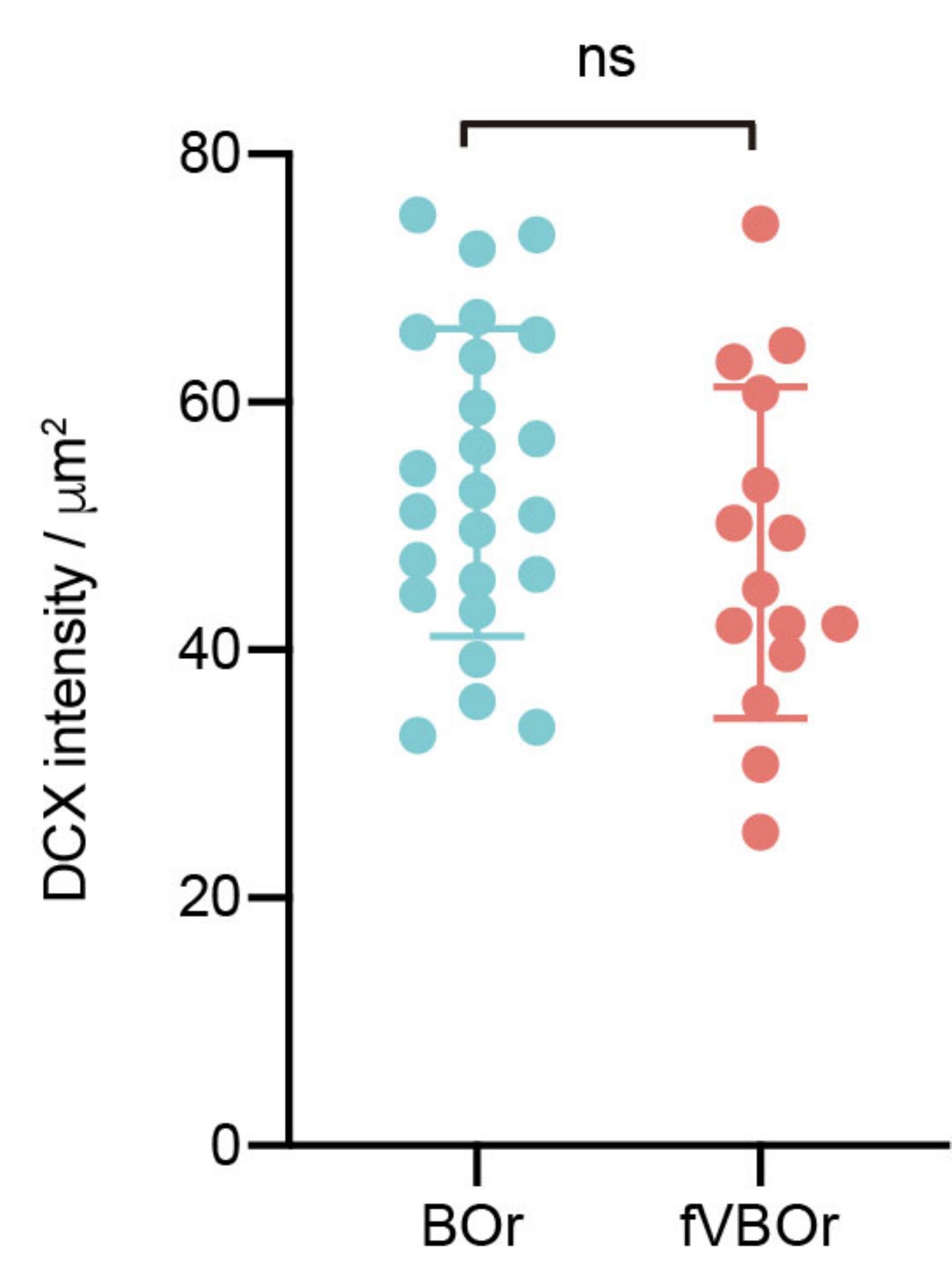

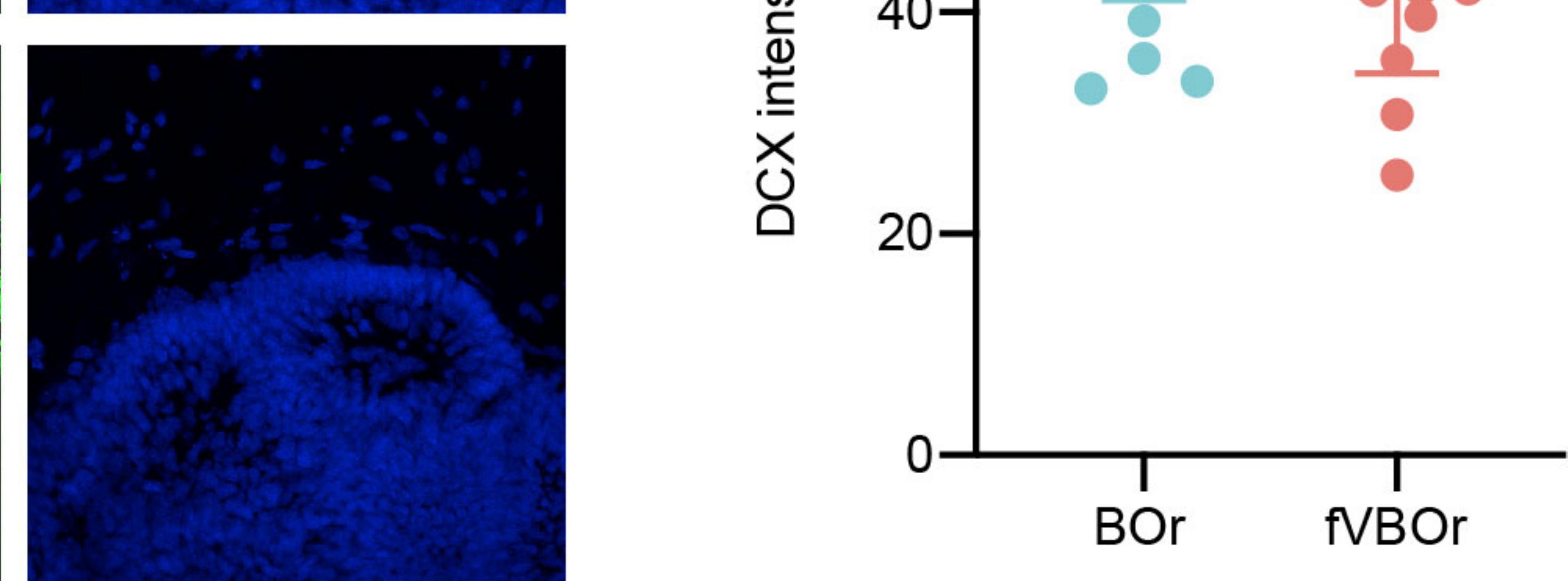



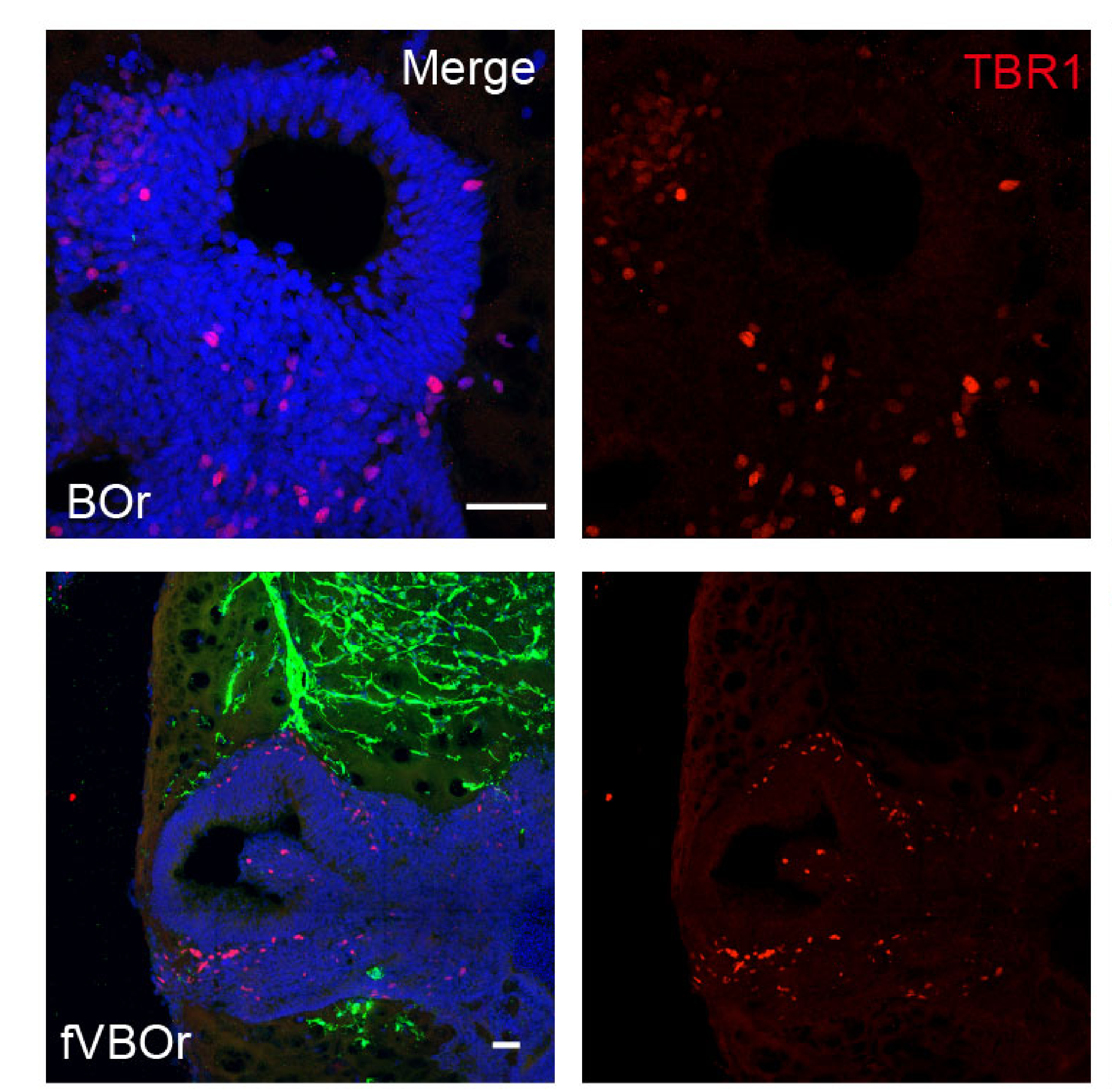

C
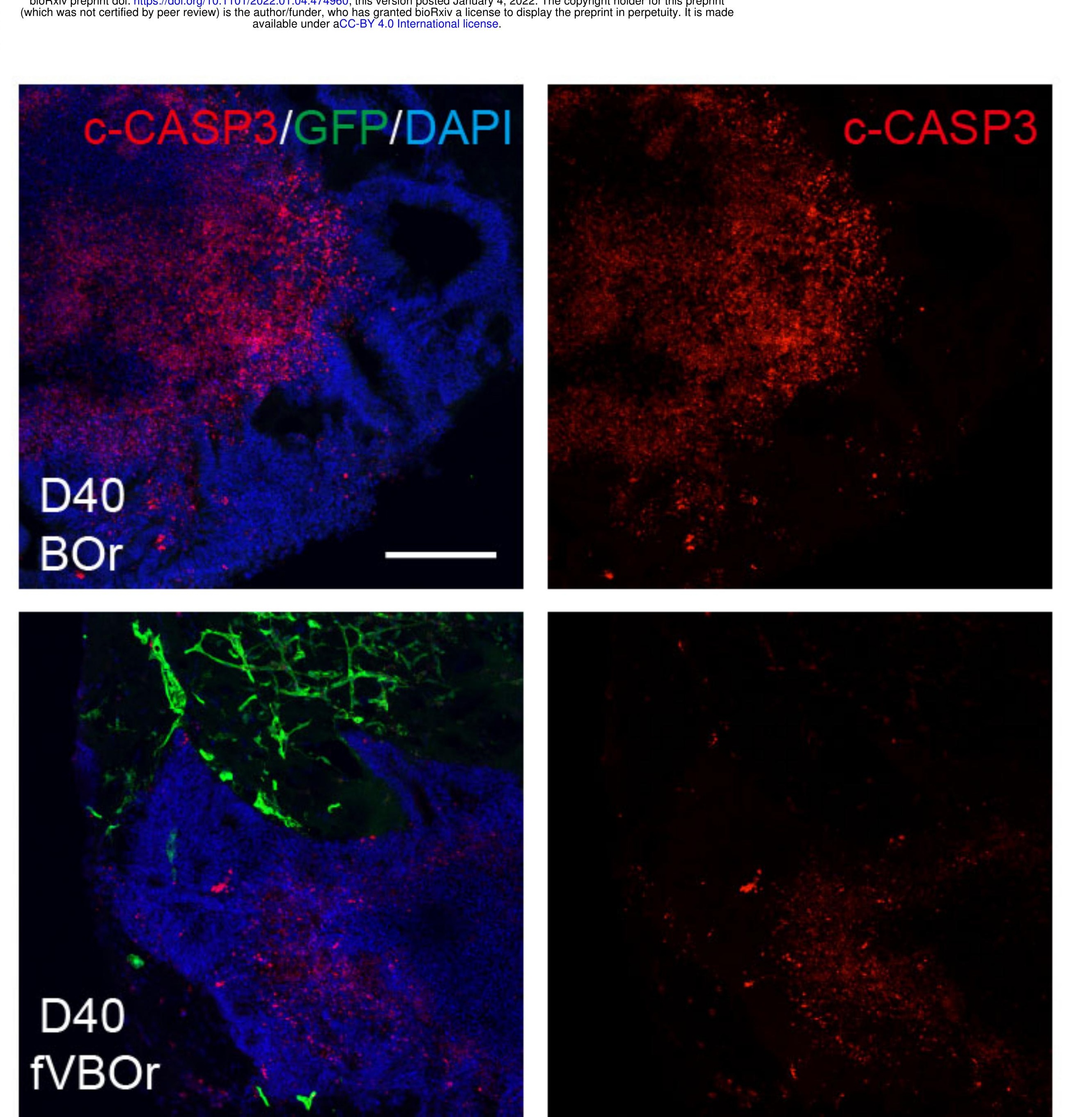
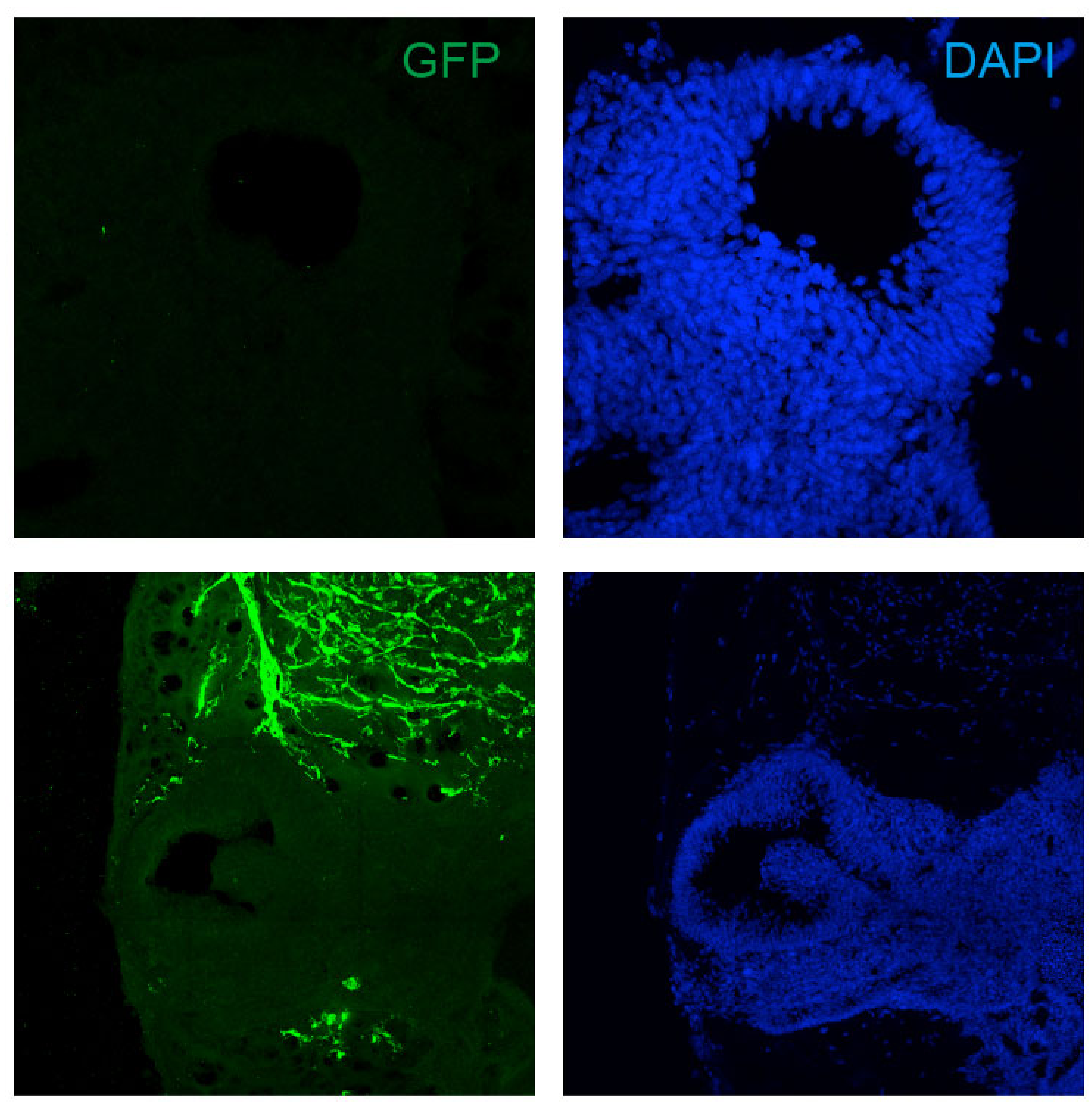

D

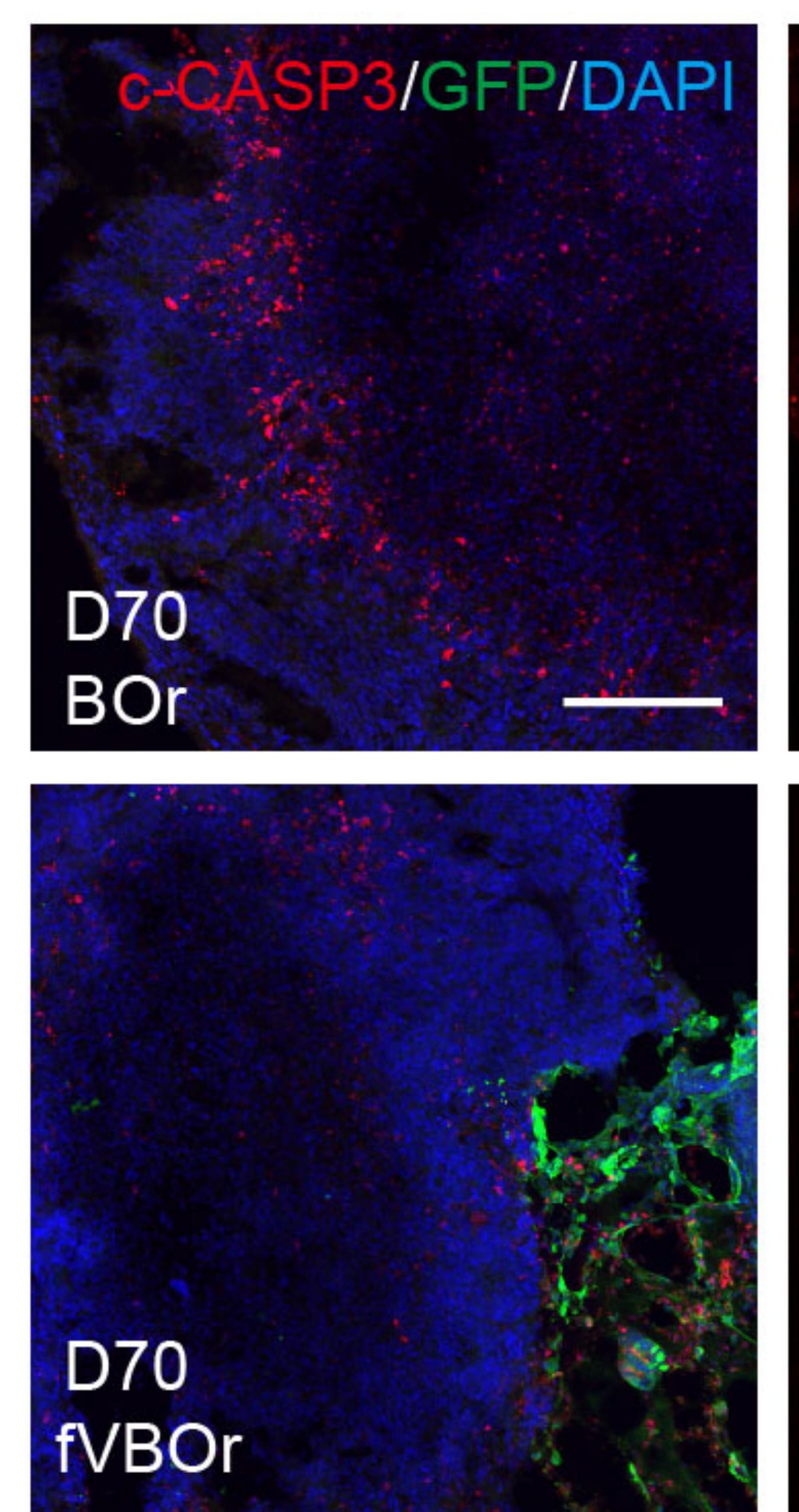

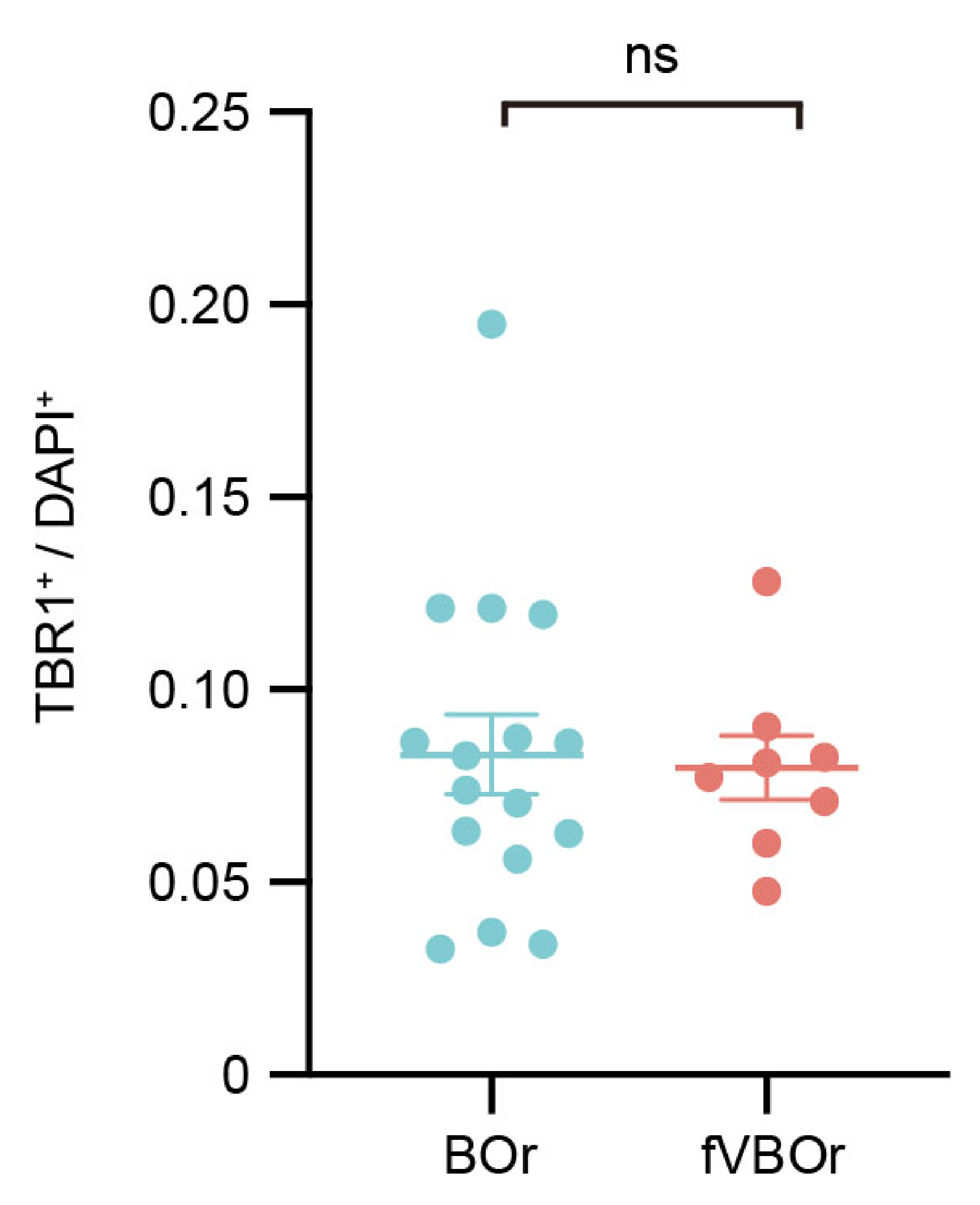

E

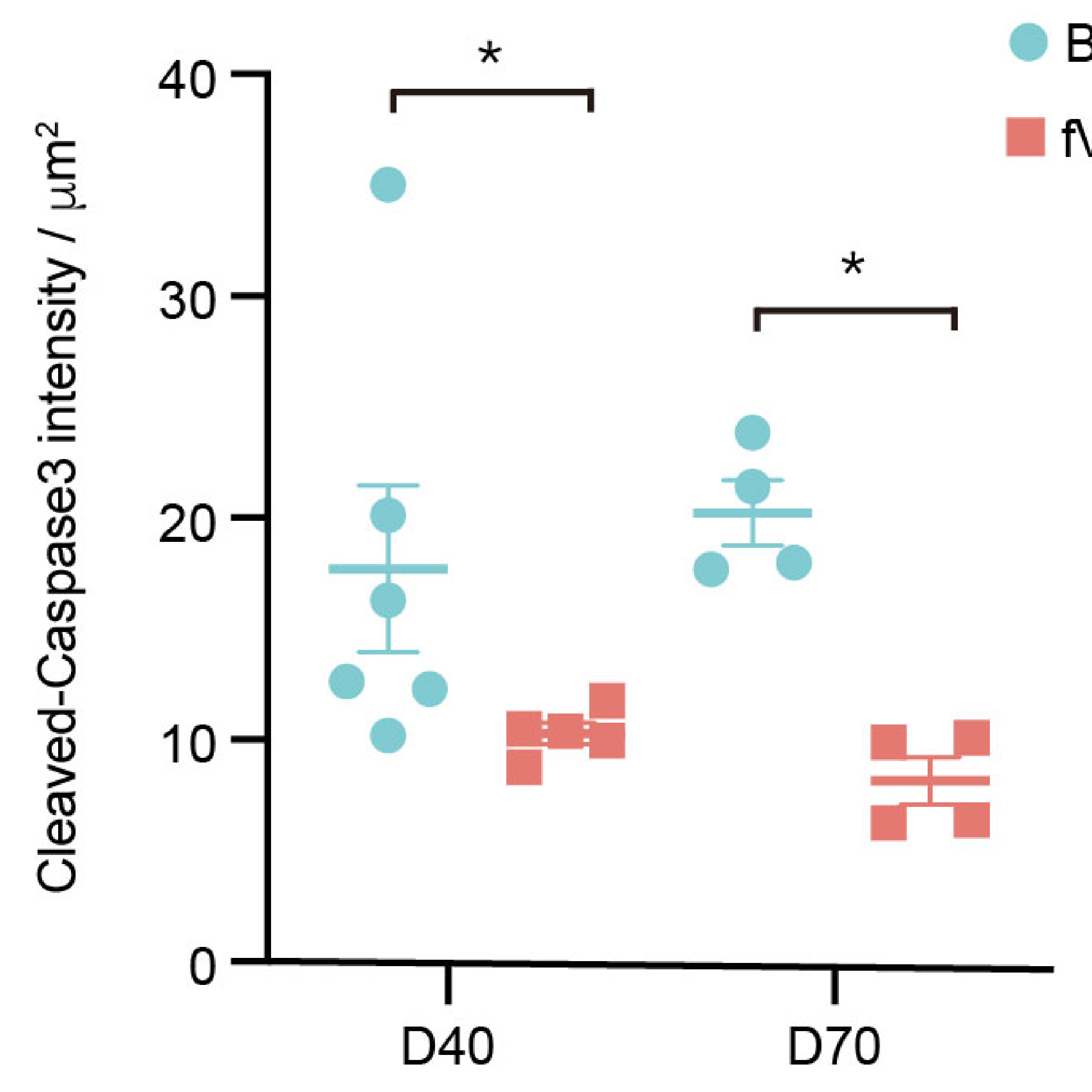

